\title{
ASYMPTOTIC FORMULAE FOR THE EIGENVALUES OF A TWO-PARAMETER ORDINARY DIFFERENTIAL EQUATION OF THE SECOND ORDER(')
}

\author{
BY
}

M. FAIERMAN

\begin{abstract}
We consider a two-point boundary value problem associated with an ordinary differential equation defined over the unit interval and containing the two parameters $\lambda$ and $\mu$. If for each real $\mu$ we denote the $m$ th eigenvalue of our system by $\lambda_{m}(\mu)$, then it is known that $\lambda_{m}(\mu)$ is real analytic in $-\infty<\mu<\infty$. In this paper we concern ourselves with the asymptotic development of $\lambda_{m}(\mu)$ as $\mu \rightarrow \infty$, and indeed obtain such a development to an accuracy determined by the coefficients of our differential equation. With suitable conditions on the coefficients of our differential equation, the asymptotic formula for $\lambda_{m}(\mu)$ may be further developed using the methods of this paper. These results may be modified so as to apply to $\lambda_{m}(\mu)$ as $\mu \rightarrow-\infty$ if the coefficients of our differential equation are also suitably modified.
\end{abstract}

1. Introduction. Here we shall consider the linear system in the two parameters $\lambda$ and $\mu$,

$$
\begin{array}{rlrl}
y^{\prime \prime}(x)+(\lambda+\mu a(x)+q(x)) y(x) & =0, & & 0 \leqq x \leqq 1, \quad{ }^{\prime}=d / d x, \\
y(0) \cos \alpha-y^{\prime}(0) \sin \alpha=0, & 0 \leqq \alpha<\pi, \\
y(1) \cos \beta-y^{\prime}(1) \sin \beta=0, & 0<\beta \leqq \pi ;
\end{array}
$$

and for the moment we shall assume that both $a(x)$ and $q(x)$ are real-valued, continuous functions in $[0,1]$. Then for each real $\mu$, the eigenvalues of $(1.1-2)$ are real and form a countably infinite set denoted by $\left\{\lambda_{n}(\mu)\right\}_{n=0}^{\infty}$, with $\lambda_{0}(\mu)<\lambda_{1}(\mu)<\cdots$, $\lim _{n \rightarrow \infty} \lambda_{n}(\mu)=\infty$, and where an eigenfunction corresponding to $\lambda_{n}(\mu)$ has precisely $n$ zeros in $(0,1)$. For $n=0,1, \ldots, \lambda_{n}(\mu)$ is a real analytic function of $\mu,-\infty<\mu<\infty$; so that in the real $(\mu, \lambda)$-plane the sets $\left\{\left(\mu, \lambda_{n}(\mu)\right),-\infty<\mu<\infty\right\}, n=0,1, \ldots$, form a countably infinite number of disjoint analytic curves called the eigenvalue curves. A great deal of literature is devoted to the study of these eigenvalue curves, and we

Received by the editors March 26, 1971.

AMS 1970 subject classifications. Primary 34B25; Secondary 34E20.

Key words and phrases. Linear system, two parameters, real-valued functions, continuous functions, eigenvalues, absolute maximum, transition points, asymptotic integration, Weber equation, parabolic cylinder function, modified Bessel equation, modified Bessel function, matching of solutions, adjacent subintervals, main equation, inverse function theorem, perturbed equation, eigenfunctions, orthogonal properties of the eigenfunctions, equations in integers.

(1) Part of this work was done while the author was a fellow of the Canadian Mathematical Congress Summer Research Institute, Montreal, 1968. This work was also supported in part by the National Research Council of Canada. 
refer to [1], [2], [3, Chapter 3], [4, Chapter 2], [5] and the references listed therein for further information.

We shall from now on in this paper assume that $a(x) \in C_{4}[0,1]$ and attains its absolute maximum in $[0,1]$ at only a finite number of points, and at each such point $a^{\prime}(x)=0, a^{\prime \prime}(x)<0$. Then in the sequel we shall always assume that $\mu$ is real and concern ourselves with the asymptotic behaviour of the eigenvalue curves as $\mu \rightarrow \infty$. Indeed, if we now agree to fix the integer $m \geqq 0$ and put $A=\sup _{0 \leqq x \leqq 1} a(x)$, then it is the purpose of this paper to prove that there are numbers $\left\{A_{i, m}\right\}_{i=1}^{3}$ such that

$$
\lambda_{m}(\mu)=-\mu A+\mu^{1 / 2} A_{1, m}+\mu^{1 / 4} A_{2, m}+A_{3, m}+o(1) \quad \text { as } \mu \rightarrow \infty .
$$

We shall also give simple methods for determining the $A_{i, m}, i=1,2,3$.

Before proceeding, we remark that if we assume $a(x) \in C_{4}[0,1]$ and attains its absolute minimum in $[0,1]$ at only a finite number of points, and at each such point $a^{\prime}(x)=0, a^{\prime \prime}(x)>0$, then the asymptotic development of $\lambda_{m}(\mu)$ as $\mu \rightarrow-\infty$ can be obtained directly once the validity of (1.3) has been established. For we need only consider the analogue of (1.3) for the system

$$
y^{\prime \prime}(x)+(\lambda+\tau b(x)+q(x)) y(x)=0, \quad 0 \leqq x \leqq 1,
$$

and (1.2), where $b(x)=-a(x), 0 \leqq x \leqq 1$, and $\tau=-\mu$.

Now in order to explain our problem in more detail, we first point out the motivation behind this paper. In. his investigation of the asymptotic behaviour of the eigenvalues associated with the generalized Hill equation, Strutt $[5, \S 13]$ reduces the problem of the asymptotic integration of this equation in an interval containing two transition points in its interior, to the problem of the asymptotic integration of this equation in two subintervals, with each containing precisely one simple transition point in its interior (see [6], [7]) and then matching his solutions in value at the meet and at the two remaining end points of the subintervals. Since in the limit the two transition points coalesce, a little reflection soon shows that the method employed by Strutt is not valid.

To show the connection between the problem considered here and the work of Strutt, we make use of the fact (see $\S 2$ ) that with $m$ as above and $\mu>0, \Lambda_{m}(\mu)$ $=\left(\lambda_{m}(\mu)+\mu A\right) / \mu^{1 / 2}$ is positive and bounded for all $\mu$ sufficiently large. Hence if $0<h<1$ and $a(h)=A$, then for each $\mu$ sufficiently large the equation $A-a(x)$ $=\mu^{-1 / 2} \Lambda_{m}(\mu)$ has in a neighbourhood of $h$ precisely two solutions (transition points) which coalesce to $h$ as $\mu \rightarrow \infty$. If $h=0$ or $h=1$, then of course we have only one transition point. Hence if we now refer to (1.1) with $\lambda=\lambda_{m}(\mu)$ as (1.1.m), then we see that an investigation of the asymptotic behaviour of $\lambda_{m}(\mu)$ as $\mu \rightarrow \infty$ will in some way involve the asymptotic integration of $(1.1 . \mathrm{m})$ in intervals containing transition points of the kind discussed by Strutt.

This last remark is best elucidated by a description of the procedure followed in this paper. If $a(h)=A$ and $0<h<1$, then we let $h$ be the midpoint of a suitably chosen closed subinterval of $[0,1]$ whose length tends to zero as $\mu \rightarrow \infty$ and such 
that for all $\mu$ sufficiently large the two transition points associated with $h$ (as explained above) are contained in the interior of this subinterval. If $a(h)=A$ and $h=0$ or $h=1$, then of course we let $h$ be the fixed end point of such a subinterval. For $\mu$ large, we add to the points of the partition of $[0,1]$, induced by the end points of the above subintervals, the points $x=0$ and $x=1$, if they are not already included; and if $a(x)=A$ in more than one point, and if $h_{1}$ and $h_{2}$ are two consecutive points for which $a\left(h_{1}\right)=a\left(h_{2}\right)=A$, then we also add to the points of our partition the point $\left(h_{1}+h_{2}\right) / 2$. Hence for each large $\mu$ we arrive at a final partition of $[0,1]$ which induces a subdivision of this interval into a finite number (independent of $\mu$ ) of closed, nonoverlapping subintervals whose end points are consecutive points of our partition, and with the property that each such subinterval contains precisely two, one, or no transition points.

Now, unlike Strutt, we strive for uniform asymptotic formulae for the solutions of $(1.1 . \mathrm{m})$ as well as their first derivatives in each of the aforementioned subintervals. This is accomplished by using the usual techniques employed in the asymptotic integration of differential equations in intervals of the kind described above. That is to say, in an interval containing transition points we approximate (1.1.m) by means of Weber's equation, express a solution in terms of a parabolic cylinder function and a correcting term, establish the integral equation for this correcting term by means of the variation-of-constants method, and from this integral equation obtain estimates for our correction; similarly for an interval not containing transition points we proceed as above, but using the modified Bessel equation and modified Bessel function in place of Weber's equation and parabolic cylinder function, respectively. For further information we refer to [8], [9], [10], [11]. If for large $\mu$ we now match our solutions in value and in value of the first derivative at the meet of two adjacent subintervals, and consider a solution in the subinterval containing $x=0$ which satisfies the first statement of (1.2), and a solution in the subinterval containing $x=1$ which satisfies the second statement of (1.2), then we arrive at a certain equation involving $\Lambda_{m}(\mu)$, which we shall call our main equation, that enables us to verify (1.3).

As can be seen from our above remarks, no great difficulty is encountered in arriving at our main equation, although the task is quite laborious. Similarly, by arguing with our main equation, it is not difficult to show that there exist numbers $A_{i, m}, i=1,2,3$, such that $\lambda_{m}(\mu)$ exhibits the asymptotic development given in (1.3). Where the difficulties do arise is in determining the $A_{i, m}$. Before pointing out these difficulties, we first wish to remark that the determination of $A_{1, m}$ depends only upon $m$ and the value of $a^{\prime \prime}(x)$ at each of the points in $[0,1]$ at which $a(x)=A$. Now let $a(x)=A$ at precisely the finite set of points $h_{r}, r=1, \ldots, p, p \geqq 1,0 \leqq h_{1}$ $<h_{2}<\cdots<h_{p} \leqq 1$, and put $b_{r}=-\frac{1}{2} \times d^{2} a\left(h_{r}\right) / d x^{2}, r=1, \ldots, p$. If $p=1$, or if $p>1$ and if for $i, j=1, \ldots, p$ and $i \neq j,\left(b_{i} / b_{j}\right)^{1 / 2}$ is not the quotient of two odd integers, then $A_{1, m}$ can be readily determined by the standard technique of investigating the asymptotic behaviour of an eigenfunction of (1.1-2) corresponding to $\lambda_{m}(\mu)$. 
Moreover, for these cases, no more than a knowledge of $A_{1, m}$ is required for the determination of $A_{2, m}$ and $A_{3, m}$. However, if $p>1$, and if for some pair $(i, j), i \neq j$, $\left(b_{i} / b_{j}\right)^{1 / 2}$ is the quotient of two odd integers, then we are immediately faced with certain difficulties. Firstly, the technique used above in determining $A_{1, m}$ is no longer applicable. Secondly, a knowledge of $A_{1, m}$ does not necessarily supply us with all the information that is required to determine $A_{2, m}$ and $A_{3, m}$. Indeed to elaborate on this second point, we shall show in the sequel that if $h_{1}>0$ and $h_{p}<1$, then a knowledge of $A_{1, m}$ always determines (1) a nonnegative integer $p^{*}$ not exceeding $(p-1),(2)$ numbers $w_{i}, i=0, \ldots, p^{*}$, which are not necessarily distinct, and (3) a set $P(m)$ of $\left(p^{*}+1\right)$ consecutive nonnegative integers containing $m$, and such that for each $N \in P(m),\left\{\lambda_{N}(\mu)+\mu A-\mu^{1 / 2} A_{1, m}\right\}$ tends to precisely one element of $B$ as $\mu \rightarrow \infty$, where $B$ is the subset of the reals whose elements are precisely the $w_{i}$. This shows that $A_{2, m}=0$; but if $p^{*}>0$ and at least two of the $w_{i}$ are distinct, then in order to determine $A_{3, m}$, we must first answer the question: how can we distinguish between different eigenvalues as $\mu \rightarrow \infty$ ?

We shall now give an outline of the work done in this paper, and in the process show how the problems stated above have been resolved. In $\$ 2$ we introduce certain preliminary results and terminology. In $\S 3$ we consider the case where $a(x)$ attains its absolute maximum in $[0,1]$ at precisely the point $h_{1}$, where $0<h_{1}<1$. Then for large $\mu$ we construct a fundamental set of solutions for (1.1.m) in each of the intervals $0 \leqq x \leqq h_{1}-H(\mu), h_{1}-H(\mu) \leqq x \leqq h_{1}+H(\mu)$, and $h_{1}+H(\mu) \leqq x \leqq 1$, where $H(\mu)=\mu^{-3 / 16} /\left(4 b_{1}\right)^{1 / 4}$; and in the manner explained above we obtain the asymptotic development of each such solution and its first derivative at the end points of its relevant interval.

In $\S 4$ we consider the case where $a(x)=A$ at precisely the points $h_{r}, r=1, \ldots, p$, $p \geqq 1,0<h_{1}<\cdots<h_{p}<1$; and for $\mu>0$ and $b_{r}$ as beforc, we put

$$
\nu_{r}(\mu)=\left\{\left(\Lambda_{m}(\mu) / 2\left(b_{r}\right)^{1 / 2}\right)-\frac{1}{2}\right\}, \quad r=1, \ldots, p .
$$

Then using the results of $\S 3$, we establish our main equation, which is just (4.1) if $p=1$, and (4.3) if $p>1$. In Theorem 4 .1 we consider the case $p=1$; and since it is known that $\lim _{\mu \rightarrow \infty} \nu_{1}(\mu)=m$, we then argue with (4.1) and the inverse function theorem to determine the $A_{i, m}$. Assuming now that $p>1$, and using the fact that the $\nu_{r}(\mu)$ are linearly related (see (4.2)) we then argue with (4.3) in Theorem 4.2 to show that as $\mu \rightarrow \infty, \nu_{r}(\mu)$ tends to a finite limit, denoted by $\nu_{r}(\infty), r=1, \ldots, p$, and at least one such limit is an integer. Now from the definition of the $\nu_{r}(\mu)$, it follows that the $\left(b_{r}\right)^{1 / 2}\left(\nu_{r}(\infty)+\frac{1}{2}\right), r=1, \ldots, p$, are all equal and $A_{1, m}=2\left(b_{r}\right)^{1 / 2}\left(\nu_{r}(\infty)+\frac{1}{2}\right)$; hence once the $\nu_{r}(\infty)$ are determined, then so is $A_{1, m}$. In Theorem 4.3 we consider the case where for $i, j=1, \ldots, p$ and $i \neq j,\left(b_{i} / b_{f}\right)^{1 / 2}$ is not the quotient of two odd integers. For this case precisely one element of the set $\left\{v_{r}(\infty)\right\}_{r=1}^{p}$ is an integer, as can be seen from the linear relationship connecting the $\nu_{r}(\infty)$. Hence we can determine the $\nu_{r}(\infty)$ from the known properties of the real zeros of the parabolic cylinder functions, since for arbitrarily large values of $\mu$ the $m$ zeros of an eigenfunction of 
(1.1-2) corresponding to $\lambda_{m}(\mu)$ which lie in the interval $(0,1)$ must all be arbitrarily near the $h_{r}$, and about each $h_{r}$ this eigenfunction may be approximated by a parabolic cylinder function. Indeed in Theorem 4.3 we show how the $\nu_{r}(\infty)$ may be determined by solving certain equations in integers. With the $v_{r}(\infty)$ determined, and using the fact that only one such element is an integer, we then argue with (4.3) and the inverse function theorem to determine $A_{2, m}$ and $A_{3, m}$.

For the remainder of $\S 4$ it is assumed that for at least one pair of integers $(i, j)$, $i \neq j,\left(b_{i} / b_{j}\right)^{1 / 2}$ is the quotient of two odd integers. Since now more than one element of the set $\left\{\nu_{r}(\infty)\right\}_{r=1}^{p}$ may be an integer, the technique used in Theorem 4.3 to determine the $\nu_{r}(\infty)$ is no longer valid. In Theorem 4.4 we show how the $\nu_{r}(\infty)$ may still be determined. Here we replace $a(x)$ in (1.1) by a suitable function $a(x, t), t>0$, chosen so as to ensure that for all $t$ sufficiently small precisely one element of the set $\left\{\nu_{r}(\infty, t)\right\}_{r=1}^{p}$ is an integer, where $\nu_{r}(\infty, t)$ is the analogue of $\nu_{r}(\infty)$ for our new system. Then arguing as in Theorem 4.3 , we determine the $v_{r}(\infty, t)$ for all $t$ sufficiently small. The $\nu_{r}(\infty)$ are then determined from the $\nu_{r}(\infty, t)$ by considering the limiting process as $t \rightarrow 0$. Also in Theorem 4.4, as in Theorem 4.3, we show how the $\nu_{r}(\infty)$ may be determined by solving certain equations in integers. With the $\nu_{r}(\infty)$ determined, we consider in Theorem 4.5 the case where only one such element is an integer; here we argue with (4.3) as before to determine $A_{2, m}$ and $A_{3, m}$. In Theorem 4.6 we state how $A_{2, m}$ and $A_{3, m}$ are determined for the case where $\left(p^{*}+1\right)$ elements of the set $\left\{\nu_{r}(\infty)\right\}_{r=1}^{p}$ are integers, and where $1 \leqq p^{*} \leqq(p-1)$; and the remainder of $\S 4$ is taken up with the proof of this theorem.

Now in order to prove Theorem 4.6, we can no longer restrict ourselves to the investigation of the asymptotic behaviour of just $\lambda_{m}(\mu)$. To see this, we introduce the $\left(p^{*}+1\right)$ numbers $w_{i}^{\prime}$, where $w_{0} \leqq w_{1} \leqq \cdots \leqq w_{p^{*}}$, which are completely determined by the $\nu_{r}(\infty)$, the $d^{j} a\left(h_{r}\right) / d x^{j}, j=2,3,4$, and the $q\left(h_{r}\right), r=1, \ldots, p$. If we denote by $B$ the subset of the reals whose elements are precisely the $w_{i}$, then we show in Lemmas 4.1, 4.2, and the first part of Theorem 4.7, that there exists the set $P(m)$ of $\left(p^{*}+1\right)$ consecutive nonnegative integers containing $m$, and such that for each $N \in P(m),\left\{\lambda_{N}(\mu)+\mu A-\mu^{1 / 2} A_{1, m}\right\}$ tends to precisely one element of $B$ as $\mu \rightarrow \infty$. This shows that $A_{2, m}=0$, and we can also determine $A_{3, m}$ if all the $w_{i}$ are equal; but if at least two of the $w_{i}$ are distinct, then we cannot determine $A_{3, m}$ until we have first shown how to distinguish between different eigenvalues. This is precisely the problem that is taken up in Theorem 4.7; indeed if we denote the set $P(m)$ by $\left\{N_{1}+j\right\}_{j=0}^{p^{*}}$, then we assert in this theorem that $M_{j}(\mu)=\lambda_{N_{1}+j}(\mu)+\mu A$ $-\mu^{1 / 2} A_{1, m}=w_{j}+o(1)$ as $\mu \rightarrow \infty, j=0, \ldots, p^{*}$. To prove this theorem, we first consider the case where the $w_{i}$ are all distinct, and establish asymptotic formulae for the eigenfunctions corresponding to $\lambda_{N}(\mu), N \in P(m)$. Then by utilizing the orthogonal properties of the eigenfunctions, we show that the $M_{,}(\infty)$ are all distinct, where $M_{j}(\infty)=\lim _{\mu \rightarrow \infty} M_{j}(\mu), j=0, \ldots, p^{*}$. Since $M_{0}(\infty) \leqq M_{1}(\infty) \leqq \cdots \leqq M_{p *}(\infty)$, our theorem is proved for this case. If the $w_{i}$ are not all distinct, we replace $q(x)$ in (1.1) by a suitable function $q(x, t), t>0$, chosen so as to ensure that for all $t$ suffi- 
ciently small, $w_{0}(t)<w_{1}(t)<\cdots<w_{p^{*}}(t)$, where $w_{i}(t)$ is the analogue of $w_{i}$ for our new system and $\lim _{t \rightarrow 0} w_{i}(t)=w_{i}, i=0, \ldots, p^{*}$. If for each $N \in P(m)$ we denote by $\lambda_{N}(\mu, t)$ the analogue of $\lambda_{N}(\mu)$ for our new system, then from above we see that for $j=0, \ldots, p^{*}, \lambda_{N_{1}+j}(\mu, t)+\mu A-\mu^{1 / 2} A_{1, m}=w_{j}(t)+o(1)$ as $\mu \rightarrow \infty$ for all $t$ sufficiently small. The proof of Theorem 4.7 is completed by considering the limiting process as $t \rightarrow 0$.

In $\S 5$ we assume that $a(x)=A$ at precisely the points $h_{r}, r=0,1, \ldots, p$, where $p \geqq 0$ and $0=h_{0}<h_{1}<\cdots<h_{p}<1$. The case $\alpha=0$ is treated in subsection 5.1 and the case $\alpha \neq 0$ in subsection 5.2. The arguments employed here are similar to those of $\S 4$.

In $\S 6$ we assume that $a(x)=A$ at precisely the points $h_{r}, r=0, \ldots,(p+1)$, where $p \geqq 0$ and $0=h_{0}<h_{1}<\cdots<h_{p+1}=1$. The case $\alpha=0, \beta=\pi$ is treated in subsection 6.1 and the case $\alpha=0, \beta \neq \pi$ in subsection 6.2 ; while the case $\alpha \neq 0, \beta \neq \pi$ is treated in subsection 6.3 and the case $\alpha \neq 0, \beta=\pi$ in subsection 6.4. The arguments employed here are similar to those of $\S 4$.

Referring to (1.3), we wish now to state that essentially five different forms of this equation appear in the analysis. In one form $A_{2, m}=0$ and $A_{3, m}$ does not depend upon $\alpha$ nor $\beta$; and this is always the case if $a(0)<A$ and $a(1)<A$. If $a(0)=A$ and $a(1)<A$, then another two forms may appear, one for the case $\alpha=0$ and another for the case $\alpha \neq 0$. Finally if $a(0)=A$ and $a(1)=A$, then two more forms may appear, one for the case $\beta=\pi$ and another for the case $\beta \neq \pi$.

We wish also to state that (1.3) is valid as an asymptotic formula, that is to say, with suitable conditions on the coefficients of the differential equation (1.1), we may use the methods of this paper to further develop this formula. Indeed we assert that $\lambda_{m}(\mu)$ can always be developed to an accuracy determined by the coefficients of our differential equation. To see this, we first observe that the asymptotic formulae for the solutions of (1.1.m) given in $\$ 3$ may be further developed for suitable $a(x)$ and $q(x)$. If for simplicity we now restrict ourselves to the discussion of the case considered in $\$ 4$, then this last remark implies that the expressions on the left-hand side of (4.1) and (4.3) may be further developed for suitable $a(x)$ and $q(x)$. Hence if $p=1$, or if $p>1$ and precisely one element of the set $\left\{\nu_{r}(\infty)\right\}_{r=1}^{p}$ is an integer, then our assertion concerning $\lambda_{m}(\mu)$ follows directly from an application of the inverse function theorem. Referring to Theorem 4.7, we see that if $p>1$ and at least two elements of the set $\left\{\nu_{r}(\infty)\right\}_{r=1}^{p}$ are integers, then our assertion concerning $\lambda_{m}(\mu)$ can also be demonstrated either by arguing directly with our eigenfunctions (if certain conditions are satisfied) or by replacing (1.1) by (4.23) and considering the limiting process. In this latter case, of course, the choice of the function $q(x, t)$ given in (4.23) will depend upon the accuracy to which we are developing $\lambda_{m}(\mu)$.

We now compare our results for system (1.1-2) with the known results for the Mathieu equation (see [12, pp. 111-112 and p. 126]). If we take $a(x)=-2 \pi^{2} \cos 2 \pi x$ and $\alpha=\beta=\pi / 2$, then the eigenvalues, $\left\{a_{n}(\mu)\right\}_{n=0}^{\infty}$, of the Sturm-Liouville problem for the even Mathieu functions are given by $a_{n}(\mu)=\pi^{-2} \lambda_{n}(\mu), n=0,1, \ldots$; hence from Theorem 4.1 we see that $a_{m}(\mu)=-2 \mu+2(2 m+1) \mu^{1 / 2}-\left(2 m^{2}+2 m+1\right) / 4+o(1)$ 
as $\mu \rightarrow \infty$. If we take $a(x)=-\left(\pi^{2} / 2\right) \cos \pi(1-x), \alpha=0$, and $\beta=\pi / 2$, then the eigenvalues, $\left\{a_{2 n+1}(\mu)\right\}_{n=0}^{\infty}$, of the Sturm-Liouville problem for the even, half-periodic Mathieu functions are given by $a_{2 n+1}(\mu)=\pi^{-2} \lambda_{n}(\mu), n=0,1, \ldots$; hence from Theorem 5.1.1 we see that $a_{2 m+1}(\mu)=-2 \mu+2(4 m+3) \mu^{1 / 2}-\left(8 m^{2}+12 m+5\right) / 4+o(1)$ as $\mu \rightarrow \infty$. If we take $a(x)=-\left(\pi^{2} / 2\right) \cos \pi(1-x)$ and $\alpha=\beta=\pi / 2$, then the eigenvalues, $\left\{a_{2 n}(\mu)\right\}_{n=0}^{\infty}$, of the Sturm-Liouville problem for the even, periodic Mathieu functions are given by $a_{2 n}(\mu)=\pi^{-2} \lambda_{n}(\mu), n=0,1, \ldots$; hence from Theorem 5.2.1 we see that $a_{2 m}(\mu)=-2 \mu+2(4 m+1) \mu^{1 / 2}-\left(8 m^{2}+4 m+1\right) / 4+o(1)$ as $\mu \rightarrow \infty$. In all cases our results are in agreement with the known results.

Finally, the author wishes to express his gratitude to Professor F. V. Atkinson for his criticisms and suggestions.

2. Preliminary results and notation. As stated in the introduction, we shall in the sequel consider the nonnegative integer $m$ fixed (unless otherwise stated) and concern ourselves in this paper with the behaviour of $\lambda_{m}(\mu)$ as $\mu \rightarrow \infty$. For $\mu>0$, we put as before $\Lambda_{m}(\mu)=\left(\lambda_{m}(\mu)+\mu A\right) / \mu^{1 / 2}$, where $A=\sup _{0 \leqq x \leqq 1} a(x)$; then there are numbers $\mu_{m}$ and $\Lambda_{m}$, both greater than one, such that for $\mu \geqq \mu_{m}, 0<\Lambda_{m}(\mu)<\Lambda_{m}$ ([3, Chapter 3], [4, p. 135]). We also remark that here and in the sequel positive roots of positive numbers are always taken.

If $r$ is a nonnegative integer, $0 \leqq h_{r} \leqq 1$, and $a\left(h_{r}\right)=A$, then we shall put

$$
\begin{aligned}
& b_{r}=-a^{(2)}\left(h_{r}\right) / 2, \quad b_{r}^{*}=\left(a^{(3)}\left(h_{r}\right) / 6 b_{r}\right)^{2} / 32, \quad b_{r}^{\dagger}=a^{(4)}\left(h_{r}\right) / 192 b_{r}, \\
& b_{r}^{\#}=-(2)^{1 / 2} a^{(3)}\left(h_{r}\right) / 12\left(4 b_{r}\right)^{3 / 4}, \quad c_{r}=b_{r}^{\#} /\left(2 b_{r}\right)^{1 / 2}, \quad d_{r}=-b_{r}^{\dagger} /\left(b_{r}\right)^{1 / 2},
\end{aligned}
$$

and for $\mu>0, \nu_{r}(\mu)=\left\{\left(\Lambda_{m}(\mu) / 2\left(b_{r}\right)^{1 / 2}\right)-\frac{1}{2}\right\}$, where $a^{(j)}\left(h_{r}\right)=d^{j} a\left(h_{r}\right) / d x^{j}, j=2,3$, 4. In the sequel we shall for convenience of notation frequently omit $\mu$ and write $\nu_{r}$ in place of $\nu_{r}(\mu)$; we also note from above that $\nu_{r}$ is bounded and greater than $-\frac{1}{2}$ for $\mu \geqq \mu_{m}$. For $t>0$ let $\Psi^{*}(t)=\Psi\left(t+\frac{1}{2}\right)-\Psi(t)$, where $\Psi(t)$ is the logarithmic derivative of the Gamma function, $\Gamma(t)$ (see Appendix I). Then for nonnegative integer $n$ and $0<\gamma<\pi$, we shall also put

$$
\begin{aligned}
G_{1,1}(r, n)= & \left(b_{r}\right)^{1 / 2}(2 n+1), \\
G_{1,2}(r, n)= & -\left[2 b_{r}^{*}\left(30 n^{2}+30 n+11\right)+6 b_{r}^{\dagger}\left(2 n^{2}+2 n+1\right)+q\left(h_{r}\right)\right], \\
G_{2,1}(r, n)= & \left(b_{r}\right)^{1 / 2}(4 n+3), \\
G_{2,2}(r, n)= & \left(32 b_{r}^{\#} / 3 \pi\right)(\Gamma(n+3 / 2) / n !)(4 n+3), \\
G_{2,3}(r, n)= & -\left[2 b_{r}^{*}\left(120 n^{2}+180 n+71\right)+6 b_{r}^{\dagger}\left(8 n^{2}+12 n+5\right)+q\left(h_{r}\right)\right. \\
& \left.\quad-\left(512 b_{r}^{*}(4 n+3) / 9 \pi^{2}\right)(\Gamma(n+3 / 2) / n !)^{2}\left\langle 4+(4 n+3) \Psi^{*}(n+1)\right\rangle\right], \\
G_{3,1}(r, n)= & \left(b_{r}\right)^{1 / 2}(4 n+1), \\
G_{3,2}(r, n, \gamma)= & (4 / \pi)(\Gamma(n+1 / 2) / n !)\left[\left(b_{r}^{\#} / 3\right)\left(32 n^{2}+16 n+3\right)+\left(\left(b_{r}\right)^{1 / 4} / 2\right) \cot \gamma\right], \\
G_{3,3}(r, n, \gamma)=-\left[2 b_{r}^{*}\left(120 n^{2}+60 n+11\right)+6 b_{r}^{\dagger}\left(8 n^{2}+4 n+1\right)+q\left(h_{r}\right)\right. & \quad\left(8 / \pi^{2}\left(b_{r}\right)^{1 / 2}\right)\left(\Gamma\left(n+\frac{1}{2}\right) / n !\right)^{2} \\
& \quad \times\left\langle\left(8 b_{r}^{\#} / 3\right)(4 n+1)-\left(\frac{1}{2}\right)\left\{\left(b_{r}^{\#} / 3\right)\left(32 n^{2}+16 n+3\right)+\left(\left(b_{r}\right)^{1 / 4} / 2\right) \cot \gamma\right\}\right. \\
\times & \left.\times\left\langle\left(b_{r}^{\#} / 3\right)\left(32 n^{2}+16 n+3\right)+\left(\left(b_{r}\right)^{1 / 4} / 2\right) \cot \gamma\right\rangle\right] .
\end{aligned}
$$




\section{Asymptotic representation of solutions.}

3.0. Introduction. In this section we shall consider the differential equation (1.1) not only under the conditions stated in the introduction, but with the further restriction that $a(x)$ attains its absolute maximum in $[0,1]$ at only the point $h_{1}$, where $0<h_{1}<1$. Furthermore, throughout this section, we shall keep the nonnegative integer $m$ fixed (see above) and consider (1.1) with $\lambda=\lambda_{m}(\mu)$. Indeed in the sequel we shall refer to (1.1) with $\lambda=\lambda_{m}(\mu)$ as (1.1.m). For convenience of notation we shall drop throughout this section also subscripts and write $h$ for $h_{1}, b$ for $b_{1}$, $c$ for $c_{1}, d$ for $d_{1}$, and $\nu$ for $\nu_{1}$ (see $\S 2$ ).

We now define on $[0,1]$ the function $\theta(x)$, obtained by putting

$$
\theta(x)=\left\{(A-a(x)) /(x-h)^{2} b\right\}
$$

if $x \neq h$, and $\theta(h)=1$. Then $\theta(x)=1+e_{1}(x-h)+X(x)(x-h)^{2}$ in $[0,1]$, where $X(h)=e_{2}, e_{1}=4 c(4 b)^{1 / 4}$, and $e_{2}=8 d(b)^{1 / 2}$; we observe that $X(x) \in C[0,1]$ and $\theta(x) \in C_{2}[0,1]$. For the remainder of this section it will be assumed that

$$
\mu \geqq \mu^{*}=\max \left\{\mu_{m},\left(h^{\dagger}(4 b)^{1 / 4}\right)^{-16}, 3\left(b^{-2}+b^{-12}\right)\left(2^{4} \Lambda_{m} \theta^{-5 / 4}\left[1+4 \theta^{\dagger}\right]\right)^{32}\right\},
$$

where $\mu_{m}$ and $\Lambda_{m}$ are defined in $\S 2$,

$$
\begin{aligned}
\theta & =\inf _{0 \leqq x \leqq 1} \theta(x), \\
\theta^{+} & =\left\{\sup _{0 \leqq x \leqq 1}\left|\theta^{\prime}(x)\right|+\sup _{0 \leqq x \leqq 1} \theta(x)\right\}, \\
h^{+} & =\min \{h / 8,(1-h) / 8\} .
\end{aligned}
$$

Finally, here we shall concern ourselves with the integration of (1.1.m), and shall proceed in the manner as explained in the introduction; we might observe that we are now dealing with a problem involving precisely two transition points, both lying in the interval $|x-h|<\mu^{-3 / 16} /(4 b)^{1 / 4}$. In subsection 3.1 we construct a fundamental set of solutions, $y_{1}(x, \mu)$ and $y_{2}(x, \mu)$, for $(1.1 . m)$ in the interval $|x-h|$ $\leqq \mu^{-3 / 16} /(4 b)^{1 / 4}$, and obtain asymptotic formulae for these solutions and their first derivatives at the end points of this interval. In subsection 3.2 we construct for all $\mu$ sufficiently large a fundamental set of solutions, $z_{3}(x, \mu)$ and $z_{4}(x, \mu)$, for (1.1.m) in the interval $h+\mu^{-3 / 16} /(4 b)^{1 / 4} \leqq x \leqq 1$, and obtain asymptotic formulae for these solutions and their first derivatives at the end points of this interval. And in subsection 3.3, we construct for all $\mu$ sufficiently large a fundamental set of solutions, $z_{1}(x, \mu)$ and $z_{2}(x, \mu)$, for $(1.1 . m)$ in the interval $0 \leqq x \leqq h-\mu^{-3 / 16} /(4 b)^{1 / 4}$, and obtain asymptotic formulae for these solutions and their first derivatives at the end points of this interval. And as explained in the introduction, it is precisely these results which will be used in the sequel to verify (1.3).

3.1. The interval $|x-h| \leqq \mu^{-3 / 16} /(4 b)^{1 / 4}$. We shall now construct a fundamental set of solutions for (1.1.m) in the interval $|x-h| \leqq \mu^{-3 / 16} /(4 b)^{1 / 4}$, and obtain asymptotic formulae for these solutions and their first derivatives at the points $x=h$ $\pm \mu^{-3 / 16} /(4 b)^{1 / 4}$; we shall also throughout this section use the results of Appendix I. 
To this end, we fix our interval by putting $\xi=(4 b)^{1 / 4}(x-h) \mu^{3 / 16}, \quad Y(\xi)=y(x)$, $\phi(\xi)=\theta(x), q_{1}(\xi)=q(x)$. Then $(1.1 . m)$ becomes

$$
\begin{aligned}
& Y^{\prime \prime}(\xi)+\left(\left(\nu+\frac{1}{2}\right) \mu^{1 / 8}-\mu^{1 / 4} \xi^{2} / 4\right) Y(\xi)=f(\xi, \mu) Y(\xi), \\
&-1 \leqq \xi \leqq 1, \quad '=d / d \xi,
\end{aligned}
$$

where $f(\xi, \mu)=-q_{1}(\xi) \mu^{-3 / 8} / 2(b)^{1 / 2}+\xi^{2} \mu^{1 / 4}(\phi(\xi)-1) / 4$. From Appendix I, we are led to consider a fundamental set of solutions of (3.1.1) in the form, $Y_{2}(\xi, \mu)$ $=$ the complex conjugate of $Y_{1}(\xi, \mu)$,

$$
\begin{aligned}
& Y_{1}(\xi, \mu)=U_{1}\left(\mu^{1 / 16} \xi, \nu\right)\left[1+u_{1}(\xi, \mu)\right], \quad-1 \leqq \xi \leqq 1, \\
& u_{1}(0, \mu)=u_{1}^{\prime}(0, \mu)=0 .
\end{aligned}
$$

We will now consider the interval $0 \leqq \xi \leqq 1$. Then on substituting (3.1.2) into (3.1.1) we have

$$
u_{1}^{\prime}(\xi, \mu)=U_{1}^{-2}\left(\mu^{1 / 16} \xi, \nu\right) \int_{0}^{\xi} f(t, \mu) U_{1}^{2}\left(\mu^{1 / 16} t, \nu\right)\left(1+u_{1}(t, \mu)\right) d t
$$

and hence $u_{1}(\xi, \mu)$ satisfies the Volterra integral equation

$$
u_{1}(\xi, \mu)=\int_{0}^{\xi} K(\xi, t, \mu) d t+\int_{0}^{\xi} K(\xi, t, \mu) u_{1}(t, \mu) d t, \quad 0 \leqq \xi \leqq 1,
$$

where

$$
\begin{aligned}
K(\xi, t, \mu)=\mu^{-1 / 16} f(t, \mu)[ & U_{1}\left(\mu^{1 / 16} t, \nu\right) U_{3}\left(\mu^{1 / 16} t, \nu\right) \\
& \left.-U_{3}\left(\mu^{1 / 16} \xi, \nu\right) U_{1}^{2}\left(\mu^{1 / 16} t, \nu\right) / U_{1}\left(\mu^{1 / 16} \xi, \nu\right)\right] .
\end{aligned}
$$

From Appendix I it is readily seen that $|K(\xi, t, \mu)| \leqq K$ in $0 \leqq t \leqq \xi \leqq 1, \mu \geqq \mu^{*}$, where $K$ is a positive constant; and so it follows that for $\mu \geqq \mu^{*}$ equation (3.1.4) has a unique solution in this interval which may be obtained by the usual method of successive approximations. We will then represent $u_{1}(\xi, \mu)$ by the convergent series $u_{1}(\xi, \mu)=\sum_{n=0}^{\infty} v_{n}(\xi, \mu), 0 \leqq \xi \leqq 1$, where $v_{0}(\xi, \mu)=\int_{0}^{\xi} K(\xi, t, \mu) d t$, and, for $n=0,1$, $2, \ldots, v_{n+1}(\xi, \mu)=\int_{0}^{\xi} K(\xi, t, \mu) v_{n}(t, \mu) d t$, and pass to the asymptotic representation of $u_{1}(1, \mu)$. Putting $s=\mu^{1 / 16} \xi$, we have, for $0 \leqq s \leqq \mu^{1 / 16}$,

$$
\begin{aligned}
v_{0}(\xi, \mu)= & -\left(\mu^{-1 / 2} / 2(b)^{1 / 2}\right) \int_{0}^{s} q_{2}(t)\left[U_{1}(t, \nu) U_{3}(t, \nu)-\frac{U_{3}(s, \nu)}{U_{1}(s, \nu)} U_{1}^{2}(t, \nu)\right] d t \\
& +c \mu^{-1 / 4} \int_{0}^{s} t^{3}\left[U_{1}(t, \nu) U_{3}(t, \nu)-\frac{U_{3}(s, \nu)}{U_{1}(s, \nu)} U_{1}^{2}(t, \nu)\right] d t \\
& +d \mu^{-1 / 2} \int_{0}^{s} t^{4}\left[U_{1}(t, \nu) U_{3}(t, \nu)-\frac{U_{3}(s, \nu)}{U_{1}(s, \nu)} U_{1}^{2}(t, \nu)\right] d t \\
& +\left(\mu^{-1 / 2} / 8(b)^{1 / 2}\right) \int_{0}^{s}\left(X_{1}(t)-e_{2}\right) t^{4}\left[U_{1}(t, \nu) U_{3}(t, \nu)-\frac{U_{3}(s, \nu)}{U_{1}(s, \nu)} U_{1}^{2}(t, \nu)\right] d t,
\end{aligned}
$$

where $q_{2}(t)=q(x), X_{1}(t)=X(x)$, and $t=(4 b \mu)^{1 / 4}(x-h)$. So from Appendix I we see that, as $\mu \rightarrow \infty, v_{0}(\xi, \mu)=O\left(\mu^{-1 / 16}\right)$, uniformly in $0 \leqq \xi \leqq 1$,

$$
\begin{aligned}
& \mathrm{R} 1 v_{0}(1, \mu)=c \mu^{-1 / 16} / 3+2 c \nu \mu^{-3 / 16}+\left[\left(3 d-4 c A_{1}(\nu)\right) / 12\right] \mu^{-1 / 4}+o\left(\mu^{-1 / 4}\right), \\
& \operatorname{Im} v_{0}(1, \mu)=c A_{2}(\nu) \mu^{-1 / 4} / 3+\left[E_{2}(\nu) q(h) / 2(b)^{1 / 2}-d B_{2}(\nu)\right] \mu^{-1 / 2}+o\left(\mu^{-1 / 2}\right) .
\end{aligned}
$$


With the same notation as above we also have

$$
\begin{aligned}
v_{1}(\xi, \mu)= & c d \mu^{-3 / 4} \int_{0}^{s} t^{4} U_{1}(t, \nu) U_{3}(t, \nu)\left(\int_{0}^{t} \tau^{3} U_{1}(\tau, \nu) U_{3}(\tau, \nu) d \tau\right) d t \\
& +c^{2} \mu^{-1 / 2} \int_{0}^{s} t^{3}\left[U_{1}(t, \nu) U_{3}(t, \nu)-\frac{U_{3}(s, \nu)}{U_{1}(s, \nu)} U_{1}^{2}(t, \nu)\right] \\
& \times\left\langle\int_{0}^{t} \tau^{3}\left[U_{1}(\tau, \nu) U_{3}(\tau, \nu)-\frac{U_{3}(t, \nu)}{U_{1}(t, \nu)} U_{1}^{2}(\tau, \nu)\right] d \tau\right\rangle d t+R(\xi, \mu),
\end{aligned}
$$

where, as $\mu \rightarrow \infty, R(\xi, \mu)=O\left(\mu^{-5 / 16}\right)$ uniformly in $0 \leqq \xi \leqq 1$ and $\operatorname{Im} R(1, \mu)=o\left(\mu^{-1 / 2}\right)$; hence, as $\mu \rightarrow \infty, v_{1}(\xi, \mu)=O\left(\mu^{-1 / 8}\right)$, uniformly in $0 \leqq \xi \leqq 1$,

$$
\begin{aligned}
\operatorname{R} 1 v_{1}(1, \mu)= & c^{2} \mu^{-1 / 8} / 18+c^{2}(8 \nu-3) \mu^{-1 / 4} / 12+o\left(\mu^{-1 / 4}\right), \\
\operatorname{Im} v_{1}(1, \mu)= & c^{2} A_{2}(\nu) \mu^{-5 / 16} / 9+2 c^{2} \nu A_{2}(\nu) \mu^{-7 / 16} / 3 \\
& +\left[c^{2}\left(9 C_{2}(\nu)-A_{1}(\nu) A_{2}(\nu)\right) / 9+c d A_{2}(\nu) / 12\right] \mu^{-1 / 2}+o\left(\mu^{-1 / 2}\right) .
\end{aligned}
$$

In a similar manner it is readily seen that, as $\mu \rightarrow \infty, v_{j}(\xi, \mu)=O\left(\mu^{-(j+1) / 16}\right)$, uniformly in $0 \leqq \xi \leqq 1, j=2, \ldots, 8$; and

$$
\begin{aligned}
& \operatorname{R} 1 v_{2}(1, \mu)=c^{3} \mu^{-3 / 16} / 162+o\left(\mu^{-1 / 4}\right), \\
& \operatorname{Im} v_{2}(1, \mu)=c^{3} A_{2}(\nu) \mu^{-3 / 8} / 54+c^{3}(8 v-3) A_{2}(\nu) \mu^{-1 / 2} / 36+o\left(\mu^{-1 / 2}\right), \\
& \operatorname{R} 1 v_{3}(1, \mu)=c^{4} \mu^{-1 / 4} / 8 \times 3^{5}+o\left(\mu^{-1 / 4}\right), \\
& \operatorname{Im} v_{3}(1, \mu)=c^{4} A_{2}(\nu) \mu^{-7 / 16} / 2 \times 3^{5}+o\left(\mu^{-1 / 2}\right), \\
& \operatorname{Im} v_{4}(1, \mu)=c^{5} A_{2}(\nu) \mu^{-1 / 2} / 8 \times 3^{6}+o\left(\mu^{-1 / 2}\right),
\end{aligned}
$$

and

$$
\operatorname{Im} v_{j}(1, \mu)=o\left(\mu^{-1 / 2}\right), \quad j=5,6,7 .
$$

From above we see that there is a $\mu_{1}$ such that for $\mu \geqq \mu_{1},\left|v_{8}(\xi, \mu)\right| \leqq K_{1} \mu^{-9 / 16}$, uniformly in $0 \leqq \xi \leqq 1$, and where $K_{1}$ is a positive constant; hence we see that for $\mu \geqq \mu_{1}$ and $0 \leqq \xi \leqq 1,\left|v_{9}(\xi, \mu)\right| \leqq K K_{1} \xi \mu^{-9 / 16}$, and in general, for $n \geqq 8,\left|v_{n}(\xi, \mu)\right|$ $\leqq K_{1} \mu^{-9 / 16}(K \xi)^{n-8} /(n-8)$ !, and so $\sum_{n=8}^{\infty}\left|v_{n}(\xi, \mu)\right| \leqq K_{1} e^{K} \mu^{-9 / 16}$. Thus referring back to our interval $|x-h| \leqq \mu^{-3 / 16} /(4 b)^{1 / 4}$, and taking here as our fundamental set of solutions for (1.1.m), $y_{1}(x, \mu)=Y_{1}(\xi, \mu), y_{2}(x, \mu)=$ the complex conjugate of $y_{1}(x, \mu)$, we have, as $\mu \rightarrow \infty$,

$$
\begin{aligned}
& y_{1}\left(h+\mu^{-3 / 16} /(4 b)^{1 / 4}, \mu\right) \\
& =\mu^{-(\nu+1) / 16} \exp \left\{-i \pi(\nu+1) / 2+\mu^{1 / 8} / 4\right\} \\
& \times\left[\left\langle1+c \mu^{-1 / 16} / 3+\left(9 v^{2}+27 \nu+18+c^{2}\right) \mu^{-1 / 8} / 18\right.\right. \\
& +c\left(27 v^{2}+405 \nu+54+c^{2}\right) \mu^{-3 / 16} / 162 \\
& +\left[c^{4} / 8 \times 3^{5}+c^{2}\left(\nu^{2}+27 \nu-7\right) / 36-c A_{1}(\nu) / 3+d / 4+(\nu+1)_{4} / 8\right] \mu^{-1 / 4} \\
& \left.+o\left(\mu^{-1 / 4}\right)\right\rangle \\
& +i\left\langle c A_{2}(\nu) \mu^{-1 / 4} / 3+c^{2} A_{2}(v) \mu^{-5 / 16} / 9+c A_{2}(\nu)\left(9 \nu^{2}+27 \nu+18+c^{2}\right) \mu^{-3 / 8} / 54\right. \\
& +c^{2} A_{2}(\nu)\left(27 \nu^{2}+405 \nu+54+c^{2}\right) \mu^{-7 / 16} / 486 \\
& +\left[c^{5} A_{2}(v) / 8 \times 3^{6}+c^{3} A_{2}(v)\left(v^{2}+27 v-7\right) / 108\right. \\
& -c^{2}\left(A_{1}(v) A_{2}(v)-9 C_{2}(v)\right) / 9+c A_{2}(v)(\nu+1)_{4} / 24+c d A_{2}(v) / 12 \\
& \left.\left.\left.-\left(d B_{2}(\nu)-q(h) E_{2}(\nu) / 2(b)^{1 / 2}\right)\right] \mu^{-1 / 2}+o\left(\mu^{-1 / 2}\right)\right\rangle\right],
\end{aligned}
$$


and similarly

$$
\begin{aligned}
& y_{1}\left(h-\mu^{-3 / 16} /(4 b)^{1 / 4}, \mu\right) \\
& =\mu^{-(\nu+1) / 16} \exp \left\{i \pi(\nu+1) / 2+\mu^{1 / 8} / 4\right\} \\
& \times\left[<1-c \mu^{-1 / 16} / 3+\left(9 v^{2}+27 \nu+18+c^{2}\right) \mu^{-1 / 8} / 18\right. \\
& -c\left(27 \nu^{2}+405 \nu+54+c^{2}\right) \mu^{-3 / 16} / 162 \\
& +\left[c^{4} / 8 \times 3^{5}+\left(\nu^{2}+27 \nu-7\right) c^{2} / 36+c A_{1}(\nu) / 3+d / 4+(\nu+1)_{4} / 8\right] \mu^{-1 / 4} \\
& +i\left\langle c A_{2}(\nu) \mu^{-1 / 4} / 3-c^{2} A_{2}(\nu) \mu^{-5 / 16} / 9+c A_{2}(\nu)\left(9 v^{2}+27 \nu+18+c^{2}\right) \mu^{-3 / 8} / 54\right. \\
& -c^{2} A_{2}(\nu)\left(27 \nu^{2}+405 \nu+54+c^{2}\right) \mu^{-7 / 16} / 486 \\
& +\left[c^{5} A_{2}(v) / 8 \times 3^{6}+c^{3} A_{2}(\nu)\left(\nu^{2}+27 \nu-7\right) / 108\right. \\
& +c^{2}\left(A_{1}(\nu) A_{2}(\nu)-9 C_{2}(\nu)\right) / 9+c A_{2}(\nu)(\nu+1)_{4} / 24+c d A_{2}(\nu) / 12 \\
& \left.\left.\left.+\left(d B_{2}(\nu)-q(h) E_{2}(\nu) / 2(b)^{1 / 2}\right)\right] \mu^{-1 / 2}+o\left(\mu^{-1 / 2}\right)\right\rangle\right]
\end{aligned}
$$

We now find the asymptotic expression for the derivative $y_{1}^{\prime}(x, \mu)$ at $x=h$ $+\mu^{-3 / 16} /(4 b)^{1 / 4}$. Put

$$
\begin{aligned}
& w_{0}(\xi, \mu)=\int_{0}^{\xi} f(t, \mu) U_{1}^{2}\left(\mu^{1 / 16} t, \nu\right) d t \\
& w_{i}(\xi, \mu)=\int_{0}^{\xi} f(t, \mu) U_{1}^{2}\left(\mu^{1 / 16} t, \nu\right) v_{i-1}(t, \mu) d t, \quad i=1,2, \ldots
\end{aligned}
$$

then from (3.1.3) and the above results we have

$$
u_{1}^{\prime}(1, \mu)=Q(\mu)+U_{1}^{-2}\left(\mu^{1 / 16}, \nu\right) \sum_{i=0}^{7} w_{i}(1, \mu),
$$

where $Q(\mu)=O\left(\mu^{-7 / 16}\right)$ as $\mu \rightarrow \infty$. It is readily seen that, as $\mu \rightarrow \infty$,

$$
\begin{aligned}
\mu^{-1 / 16} w_{0}(1, \mu)= & \mu^{-(\nu+1) / 8} \exp \left\{-i \pi(\nu+1)+\mu^{1 / 8} / 2\right\} \\
& \times\left[\left\langle c \mu^{-1 / 8}+O\left(\mu^{-1 / 4}\right)\right\rangle+i\left\langle O\left(\mu^{-1 / 2}\right)\right\rangle\right], \\
\mu^{-1 / 16} w_{1}(1, \mu)= & \mu^{-(\nu+1) / 8} \exp \left\{-i \pi(\nu+1)+\mu^{1 / 8} / 2\right\} \\
& \times\left[\left\langle c^{2} \mu^{-3 / 16} / 3+O\left(\mu^{-1 / 4}\right)\right\rangle+i\left\langle c^{2} A_{2}(\nu) \mu^{-3 / 8} / 3+O\left(\mu^{-1 / 2}\right)\right\rangle\right], \\
\mu^{-1 / 16} w_{2}(1, \mu)= & \mu^{-(\nu+1) / 8} \exp \left\{-i \pi(\nu+1)+\mu^{1 / 8} / 2\right\} \\
& \times\left[O\left(\mu^{-1 / 4}\right)+i\left\langle c^{3} A_{2}(\nu) \mu^{-7 / 16} / 9+O\left(\mu^{-1 / 2}\right)\right\rangle\right],
\end{aligned}
$$

and

$\mathrm{R} 1\left\{\mu^{-1 / 16} w_{i}(1, \mu)\right\}=O\left(\mu^{-1 / 4}\right), \quad \operatorname{Im}\left\{\mu^{-1 / 16} w_{i}(1, \mu)\right\}=O\left(\mu^{-1 / 2}\right), \quad i=3, \ldots, 7$

thus

$$
\begin{aligned}
u_{1}^{\prime}(1, \mu)=\mu^{1 / 16} & {\left[\left\langle c \mu^{-1 / 8}+c^{2} \mu^{-3 / 16} / 3+O\left(\mu^{-1 / 4}\right)\right\rangle\right.} \\
& \left.+i\left\langle c^{2} A_{2}(\nu) \mu^{-3 / 8} / 3+c^{3} A_{2}(\nu) \mu^{-7 / 16} / 9+O\left(\mu^{-1 / 2}\right)\right\rangle\right]
\end{aligned}
$$


Hence we see that, as $\mu \rightarrow \infty$,

$$
\begin{aligned}
& y_{1}^{\prime}\left(h+\mu^{-3 / 16} /(4 b)^{1 / 4}, \mu\right) \\
& =\frac{1}{2}(4 b)^{1 / 4} \mu^{-(\nu-4) / 16} \exp \left\{-i \pi(\nu+1) / 2+\mu^{1 / 8} / 4\right\} \\
& \times\left[<1+c \mu^{-1 / 16} / 3+\left(9 \nu^{2}-9 \nu-18+c^{2}\right) \mu^{-1 / 8} / 18\right. \\
& +c\left(27 \nu^{2}+297 \nu+270+c^{2}\right) \mu^{-3 / 16} / 162 \\
& +\left[c^{4} / 8 \times 3^{5}+c^{2}\left(\nu^{2}+23 \nu+13\right) / 36-c A_{1}(\nu) / 3+d / 4+(\nu-4)(\nu+1)_{3} / 8\right] \mu^{-1 / 4} \\
& +i\left\langle c A_{2}(\nu) \mu^{-1 / 4} / 3+c^{2} A_{2}(\nu) \mu^{-5 / 16} / 9+c A_{2}(\nu)\left(9 v^{2}-9 \nu-18+c^{2}\right) \mu^{-3 / 8} / 54\right. \\
& +c^{2} A_{2}(\nu)\left(27 \nu^{2}+297 \nu+270+c^{2}\right) \mu^{-7 / 16} / 486 \\
& +\left[c^{5} A_{2}(\nu) / 8 \times 3^{6}+c^{3} A_{2}(\nu)\left(\nu^{2}+23 \nu+13\right) / 108\right. \\
& \quad-c^{2}\left(A_{1}(v) A_{2}(\nu)-9 C_{2}(\nu)\right) / 9+c A_{2}(\nu)(\nu-4)(\nu+1)_{3} / 24+c d A_{2}(\nu) / 12 \\
& \left.\left.\left.\quad-\left(d B_{2}(\nu)-q(h) E_{2}(v) / 2(b)^{1 / 2}\right)\right] \mu^{-1 / 2}+o\left(\mu^{-1 / 2}\right)\right\rangle\right],
\end{aligned}
$$

and similarly

$$
\begin{aligned}
& y_{1}^{\prime}\left(h-\mu^{-3 / 16} /(4 b)^{1 / 4}, \mu\right) \\
& =-\frac{1}{2}(4 b)^{1 / 4} \mu^{-(\nu-4) / 16} \exp \left\{i \pi(\nu+1) / 2+\mu^{1 / 8} / 4\right\} \\
& \times\left[\left\langle1-c \mu^{-1 / 16} / 3+\left(9 \nu^{2}-9 \nu-18+c^{2}\right) \mu^{-1 / 8} / 18\right.\right. \\
& -c\left(27 \nu^{2}+297 \nu+270+c^{2}\right) \mu^{-3 / 16} / 162 \\
& +\left[c^{4} / 8 \times 3^{5}+c^{2}\left(\nu^{2}+23 \nu+13\right) / 36+c A_{1}(\nu) / 3+d / 4+(\nu-4)(\nu+1)_{3} / 8\right] \mu^{-1 / 4} \\
& \left.+o\left(\mu^{-1 / 4}\right)\right\rangle \\
& +i\left\langle c A_{2}(\nu) \mu^{-1 / 4} / 3-c^{2} A_{2}(\nu) \mu^{-5 / 16} / 9+c A_{2}(\nu)\left(9 \nu^{2}-9 \nu-18+c^{2}\right) \mu^{-3 / 8} / 54\right. \\
& -c^{2} A_{2}(\nu)\left(27 \nu^{2}+297 \nu+270+c^{2}\right) \mu^{-7 / 16} / 486 \\
& +\left[c^{5} A_{2}(v) / 8 \times 3^{6}+c^{3} A_{2}(v)\left(v^{2}+23 \nu+13\right) / 108\right. \\
& +c^{2}\left(A_{1}(\nu) A_{2}(\nu)-9 C_{2}(\nu)\right) / 9+c A_{2}(\nu)(\nu-4)(\nu+1)_{3} / 24+c d A_{2}(\nu) / 12 \\
& \left.\left.\left.+\left(d B_{2}(\nu)-q(h) E_{2}(\nu) / 2(b)^{1 / 2}\right)\right] \mu^{-1 / 2}+o\left(\mu^{-1 / 2}\right)\right\rangle\right] .
\end{aligned}
$$

3.2. The interval $\left(h+\mu^{-3 / 16} /(4 b)^{1 / 4}\right) \leqq x \leqq 1$. We shall now construct a fundamental set of solutions for (1.1.m) in the interval $\left(h+\mu^{-3 / 16} /(4 b)^{1 / 4}\right) \leqq x \leqq 1$ for all sufficiently large values of $\mu$ and obtain asymptotic formulae for these solutions and their first derivatives at the points $x=h+\mu^{-3 / 16} /(4 b)^{1 / 4}$ and $x=1$; we shall also throughout this section use the results of Appendix II. To this end put $x_{1}(\mu)$ $=h+\mu^{-3 / 16} /(4 b)^{1 / 4}, x_{2}(\mu)=h+\mu^{-1 / 16} /(4 b)^{1 / 4}$, and for each $\mu$, let $x_{0}(\mu)$ be the unique point such that $b\left(x_{0}(\mu)-h\right)^{2} \theta\left(x_{0}(\mu)\right)=\mu^{-1 / 2} \Lambda_{m}(\mu), h<x_{0}(\mu)<x_{1}(\mu)$; and for the interval $x_{0}(\mu) \leqq x \leqq 1$, put $r(x, \mu)=(x-h)^{2} \theta(x)-\mu^{-1 / 2} \Lambda_{m}(\mu) / b, I(x, \mu)$ $=\int_{x_{0}(\mu)}^{x} r^{1 / 2}(t, \mu) d t$, and $w(x, \mu)=(4 b)^{1 / 4} I^{1 / 2}(x, \mu)$; and for the interval $x_{1}(\mu)$ $\leqq x \leqq 1$, put $f(x, \mu)=q(x)+\left(w^{\prime}(x, \mu)\right)^{1 / 2} d^{2}\left[\left(w^{\prime}(x, \mu)\right)^{-1 / 2}\right] / d x^{2}, \quad '=d / d x$. Writing (1.1.m) in the form

$$
\begin{array}{r}
y^{\prime \prime}(x)+\left(\mu^{1 / 2} \Lambda_{m}(\mu)-\mu b(x-h)^{2} \theta(x)+q(x)-f(x, \mu)\right) y(x)=-f(x, \mu) y(x), \\
x_{1}(\mu) \leqq x \leqq 1,
\end{array}
$$

we are led from Appendix II to consider a solution $z_{3}(x, \mu)$ of (3.2.1) in the form

$$
\begin{aligned}
z_{3}(x, \mu) & =\left(w^{\prime}(x, \mu)\right)^{-1 / 2} U_{1}\left(\mu^{1 / 4} w(x, \mu)\right)\left(1+u_{1}(x, \mu)\right), \\
u_{1}\left(x_{1}(\mu), \mu\right) & =u_{1}^{\prime}\left(x_{1}(\mu), \mu\right)=0 .
\end{aligned}
$$


Substituting (3.2.2) into (3.2.1) we have

$$
\begin{aligned}
& u_{1}^{\prime}(x, \mu) \\
& \quad=-w^{\prime}(x, \mu) U_{1}^{-2}\left(\mu^{1 / 4} w(x, \mu)\right) \int_{x_{1}(\mu)}^{x} f(t, \mu)\left(w^{\prime}(t, \mu)\right)^{-1} U_{1}^{2}\left(\mu^{1 / 4} w(t, \mu)\right) d t
\end{aligned}
$$

and hence $u_{1}(x, \mu)$ satisfies the Volterra integral equation

$$
u_{1}(x, \mu)=\int_{x_{1}(\mu)}^{x} K(x, t, \mu) d t+\int_{x_{1}(\mu)}^{x} K(x, t, \mu) u_{1}(t, \mu) d t, \quad x_{1}(\mu) \leqq x \leqq 1,
$$

where

$$
\begin{aligned}
K(x, t, \mu)= & -\mu^{-1 / 4} f(t, \mu)\left(w^{\prime}(t, \mu)\right)^{-1} \\
& \times\left[U_{1}\left(\mu^{1 / 4} w(t, \mu)\right) U_{3}\left(\mu^{1 / 4} w(t, \mu)\right)\right. \\
& \left.\quad-U_{3}\left(\mu^{1 / 4} w(x, \mu)\right) U_{1}^{2}\left(\mu^{1 / 4} w(t, \mu)\right) / U_{1}\left(\mu^{1 / 4} w(x, \mu)\right)\right] .
\end{aligned}
$$

Since

$$
\begin{array}{r}
f(t, \mu) / w^{\prime}(t, \mu)=\left(8(4 b)^{1 / 4}\right)^{-1}\left[I ^ { 1 / 2 } ( t , \mu ) \left\langle16(r(t, \mu))^{-1 / 2} q(t)+5(r(t, \mu))^{-5 / 2}\right.\right. \\
\left.\times\left(r^{\prime}(t, \mu)\right)^{2}-4(r(t, \mu))^{-3 / 2} r^{\prime \prime}(t, \mu)\right\rangle \\
\left.-3(r(t, \mu))^{1 / 2}(I(t, \mu))^{-3 / 2}\right], \\
=d / d t
\end{array}
$$

and for $x_{1}(\mu) \leqq x \leqq 1$ and $\mu \geqq \mu^{*}, a_{1}(t-h)^{2} \leqq r(t, \mu) \leqq a_{2}(t-h)^{2}, a_{3}(t-h)^{2} \leqq I(t, \mu)$ $\leqq a_{4}(t-h)^{2}$, and $\left|r^{\prime}(t, \mu)\right| \leqq a_{5}(t-h),\left|r^{\prime \prime}(t, \mu)\right| \leqq a_{6}$, where $a_{i}$ is a positive constant $i=1, \ldots, 6$, we see from Appendix II that $|K(x, t, \mu)| \leqq R(t, \mu)=B \mu^{-1 / 4}(h-t)^{-2}$, in $x_{1}(\mu) \leqq t \leqq x \leqq 1, \mu \geqq \mu^{*}$, and where $B$ is a positive constant, and where we also observe that $\int_{x_{1}(\mu)}^{1} R(t, \mu) d t \leqq B^{\dagger}$ for $\mu \geqq \mu^{*}$, where $B^{\dagger}$ is a positive constant. Hence it follows that for each $\mu$ equation (3.2.4) has a unique solution in this interval which may be obtained by the usual method of successive approximations. We will then represent $u_{1}(x, \mu)$ by the convergent series $u_{1}(x, \mu)=\sum_{n=0}^{\infty} v_{n}(x, \mu), x_{1}(\mu) \leqq x \leqq 1$, where $v_{0}(x, \mu)=\int_{x_{1}(\mu)}^{x} K(x, t, \mu) d t$, and for $n=0,1, \ldots$,

$$
v_{n+1}(x, \mu)=\int_{x_{1}(\mu)}^{x} K(x, t, \mu) v_{n}(t, \mu) d t
$$

and pass to the asymptotic representation of $u_{1}(1, \mu)$.

First, it is readily deducible that, as $\mu \rightarrow \infty$,

$$
\begin{aligned}
2(x-h)^{-2} I(x, \mu)= & 1-(b)^{-1 / 2}(\nu+1 / 2)(x-h)^{-2} \\
& \times\left\langle 1+2 \log (x-h)+\log 2+(\log \mu) / 2-\log \left((\nu+1 / 2) /(b)^{1 / 2}\right)\right\rangle \mu^{-1 / 2} \\
& +e_{1}(x-h) / 3+(\nu+1 / 2)^{2}(x-h)^{-4} \mu^{-1} / 2 b \\
& +e_{1}(\nu+1 / 2)(x-h)^{-1} \mu^{-1 / 2} /(b)^{1 / 2}-\left(e_{1}^{2}-4 e_{2}\right)(x-h)^{2} / 16 \\
& +\left((\nu+1 / 2) /(b)^{1 / 2}\right)^{3}(x-h)^{-6} \mu^{-3 / 2} / 4+o\left((x-h)^{2}\right),
\end{aligned}
$$


uniformly in $x_{1}(\mu) \leqq x \leqq x_{2}(\mu)$;

$$
\begin{gathered}
(b)^{1 / 2} I(1, \mu)=\int_{h}^{1}(A-a(x))^{1 / 2} d x-((\nu+1 / 2) / 4) \mu^{-1 / 2} \log \mu \\
-\left\langle((\nu+1 / 2) / 2) \int_{h}^{1} \theta^{-3 / 2}(x) \theta^{\prime}(x) \log (x-h) d x\right. \\
\quad+(\nu+1 / 2)(1-h)(b /(A-a(1)))^{1 / 2} \log (1-h)+((\nu+1 / 2) / 2) \\
\quad \times[1-\log (\nu+1 / 2)+\log 2+(\log b) / 2]\rangle \mu^{-1 / 2} \\
+o\left(\mu^{-3 / 4}\right) ; \quad \\
5(r(x, \mu))^{-5 / 2}\left(r^{\prime}(x, \mu)\right)^{2}-4(r(x, \mu))^{-3 / 2} r^{\prime \prime}(x, \mu)+16(r(x, \mu))^{-1 / 2} q(x) \\
=12(x-h)^{-3}+76\left((\nu+1 / 2) /(b)^{1 / 2}\right)(x-h)^{-5} \mu^{-1 / 2}+O\left((x-h)^{-2}\right),
\end{gathered}
$$

uniformly in $x_{1}(\mu) \leqq x \leqq x_{2}(\mu)$; and

$$
\begin{aligned}
3(r(x, \mu))^{1 / 2} I^{-2}(x, \mu)= & 12(x-h)^{-3}+12\left((\nu+1 / 2) /(b)^{1 / 2}\right) \\
& \times\left\langle 1+4 \log (x-h)+2 \log 2+\log \mu-2 \log \left((\nu+1 / 2) /(b)^{1 / 2}\right)\right\rangle \\
& \times \mu^{-1 / 2}(x-h)^{-5}+O\left((x-h)^{-2}\right),
\end{aligned}
$$

uniformly in $x_{1}(\mu) \leqq x \leqq x_{2}(\mu)$.

We will now proceed with the calculations for $v_{0}(1, \mu)$. First, put

$$
I_{1}(x, \mu)=-\mu^{-1 / 4} \int_{x_{1}(\mu)}^{x} U_{1}\left(\mu^{1 / 4} w(t, \mu)\right) U_{3}\left(\mu^{1 / 4} w(t, \mu)\right)\left(f(t, \mu) / w^{\prime}(t, \mu)\right) d t ;
$$

then, for $x_{1}(\mu) \leqq x \leqq x_{2}(\mu)$,

$$
\begin{aligned}
I_{1}(x, \mu)= & -\left(\mu^{-1 / 2} / 32(b)^{1 / 2}\right) \int_{x_{1}(u)}^{x} 8(4 b)^{1 / 4} I^{-1 / 2}(t, \mu)\left(f(t, \mu) / w^{\prime}(t, \mu)\right) d t \\
& -\mu^{-1 / 4} \int_{x_{1}(\mu)}^{x}\left[U_{1}\left(\mu^{1 / 4} w(t, \mu)\right) U_{3}\left(\mu^{1 / 4} w(t, \mu)\right)-\frac{1}{2}\left(\mu^{1 / 4} w(t, \mu)\right)^{-1}\right] \\
& \times\left(f(t, \mu) / w^{\prime}(t, \mu)\right) d t .
\end{aligned}
$$

Hence from above and Appendix II we see, as $\mu \rightarrow \infty$,

$$
\begin{aligned}
I_{1}(x, \mu)= & -\left(\mu^{-1 / 2} / 32(b)^{1 / 2}\right) \\
& \times\left[\int_{x_{1}(\mu)}^{x}\left[12(t-h)^{-3}+76(\nu+1 / 2)(t-h)^{-5} \mu^{-1 / 2} /(b)^{1 / 2}\right] d t\right. \\
& \left.+3\left(I^{-1}(x, \mu)-I^{-1}\left(x_{1}(\mu), \mu\right)\right)\right]+o\left(\mu^{-1 / 4}\right),
\end{aligned}
$$

uniformly in $x_{1}(\mu) \leqq x \leqq x_{2}(\mu)$; thus $I_{1}(x, \mu)=O\left(\mu^{-1 / 4} \log \mu\right)$, uniformly in $x_{1}(\mu) \leqq x$ $\leqq x_{2}(\mu)$, and

$$
\begin{aligned}
& I_{1}\left(x_{2}(\mu), \mu\right) \\
& \quad=(3 / 32)(\nu+1 / 2) \mu^{-1 / 4} \log \mu-((\nu+1 / 2) / 8)[13+6 \log (\nu+1 / 2)] \mu^{-1 / 4}+o\left(\mu^{-1 / 4}\right) .
\end{aligned}
$$


For $x_{2}(\mu) \leqq x \leqq 1$, it is readily observed that $I_{1}(x, \mu)=I_{1}\left(x_{2}(\mu), \mu\right)+o\left(\mu^{-1 / 4}\right)$ as $\mu \rightarrow \infty$, uniformly in $x_{2}(\mu) \leqq x \leqq 1$. Now put

$$
\begin{aligned}
I_{2}(x, \mu)= & \mu^{-1 / 4}\left(U_{3}\left(\mu^{1 / 4} w(x, \mu)\right) / U_{1}\left(\mu^{1 / 4} w(x, \mu)\right)\right) \\
& \times \int_{x_{1}(\mu)}^{x} U_{1}^{2}\left(\mu^{1 / 4} w(t, \mu)\right)\left(f(t, \mu) / w^{\prime}(t, \mu)\right) d t ;
\end{aligned}
$$

then from above and Appendix II we see that there is a $\mu_{1}$ such that, for $\mu \geqq \mu_{1}$, $\left|I_{2}(x, \mu)\right| \leqq B_{1} \mu^{-1 / 2} F(x, \mu), x_{1}(\mu) \leqq x \leqq 1$, where $B_{1}$ is a positive constant, and

$$
F(x, \mu)=\exp \left\{-2(b \mu)^{1 / 2} I(x, \mu)\right\} \int_{x_{1}(\mu)}^{x} \exp \left\{2(b \mu)^{1 / 2} I(t, \mu)\right\}(t-h)^{-3} d t .
$$

Integration by parts shows that $F(1, \mu)=O\left(\mu^{-1 / 2}\right)$ as $\mu \rightarrow \infty$; and also we see that $\sup _{x_{1}(\mu) \leqq x \leqq 1} F(x, \mu)=O\left(\mu^{1 / 4}\right)$ as $\mu \rightarrow \infty$. Therefore, as $\mu \rightarrow \infty, I_{2}(x, \mu)=O\left(\mu^{-1 / 4}\right)$, uniformly in $x_{1}(\mu) \leqq x \leqq 1$, and $I_{2}(1, \mu)=O\left(\mu^{-1}\right)$. Hence since $v_{0}(x, \mu)=I_{1}(x, \mu)$ $+I_{2}(x, \mu)$, we have, as $\mu \rightarrow \infty, v_{0}(x, \mu)=O\left(\mu^{-1 / 4} \log \mu\right)$, uniformly in $x_{1}(\mu) \leqq x \leqq 1$, and

$$
v_{0}(1, \mu)=(3 / 32)\left(\nu+\frac{1}{2}\right) \mu^{-1 / 4} \log \mu-\left(\left(\nu+\frac{1}{2}\right) / 8\right)\left(13+6 \log \left(\nu+\frac{1}{2}\right)\right) \mu^{-1 / 4}+o\left(\mu^{-1 / 4}\right) .
$$

From the above and Appendix II we see that there is a $\mu_{2}$ such that for $\mu \geqq \mu_{2}$, $|K(x, t, \mu)| \leqq B_{2} \mu^{-1 / 2}(t-h)^{-3}$ in $x_{1}(\mu) \leqq t \leqq x \leqq 1,\left|v_{0}(t, \mu)\right| \leqq B_{3} \mu^{-1 / 4} \log \mu$ in $x_{1}(\mu)$ $\leqq t \leqq 1$, and where $B_{2}$ and $B_{3}$ are positive constants; hence for $\mu \geqq \mu_{2}$ and $x_{1}(\mu) \leqq x \leqq 1$,

$$
\left|v_{1}(x, \mu)\right| \leqq B_{3} \mu^{-1 / 4} \log \mu\left(B_{2} \mu^{-1 / 2} \int_{x_{1}(u)}^{x}(t-h)^{-3} d t\right)=B_{3} J(x, \mu) \mu^{-1 / 4} \log \mu,
$$

and for $n \geqq 2$,

$$
\left|v_{n}(x, \mu)\right| \leqq B_{3} \mu^{-1 / 4} \log \mu J^{n}(x, \mu) / n !
$$

and, therefore,

$$
\sum_{n=1}^{\infty}\left|v_{n}(x, \mu)\right| \leqq B_{3} \mu^{-1 / 4} \log \mu\left(e^{J(x, \mu)}-1\right)=O\left(\mu^{-3 / 8} \log \mu\right) \quad \text { as } \mu \rightarrow \infty,
$$

uniformly in $x_{1}(\mu) \leqq x \leqq 1$. We thus have, as $\mu \rightarrow \infty$,

$$
\begin{aligned}
& z_{3}\left(h+\mu^{-3 / 16} /(4 b)^{1 / 4}, \mu\right) \\
& =\left(\Gamma(3 / 4) /(\pi)^{1 / 2}(4 b)^{1 / 8}\right) F_{1}(\mu) \mu^{-1 / 16} \\
& \quad \times\left[1+c \mu^{1 / 16} / 3+\left(9 \nu^{2}+27 \nu+18+c^{2}\right) \mu^{-1 / 8} / 18\right. \\
& \quad+c\left(27 \nu^{2}+405 \nu+54+c^{2}\right) \mu^{-3 / 16} / 162+(3 / 64)(2 \nu+1) \mu^{-1 / 4} \log \mu \\
& \left.\quad+H_{1}(\mu) \mu^{-1 / 4}+o\left(\mu^{-1 / 4}\right)\right], \\
& \quad z_{3}(1, \mu)=\left(\Gamma(3 / 4) /(2 \pi)^{1 / 2}\right)(A-a(1))^{-1 / 4} G_{1}(\mu) \mu^{-1 / 4}\left(J_{1}(\mu)+o\left(\mu^{-1 / 4}\right)\right),
\end{aligned}
$$


where

$$
\begin{aligned}
F_{1}(\mu)= & \mu^{-v / 16} \exp \left\{-\left(\nu+\frac{1}{2}\right) / 2+\mu^{1 / 8} / 4+\left(\left(\nu+\frac{1}{2}\right) / 2\right) \log \left(\nu+\frac{1}{2}\right)\right\} \\
G_{1}(\mu)= & \mu^{-v / 4}\left((4 b)^{1 / 4} \exp \left\{(1-h)(b /(A-a(1)))^{1 / 2} \log (1-h)\right\}\right)^{-(\nu+1 / 2)} \\
& \times \exp \left\{\left(\left(\nu+\frac{1}{2}\right) / 2\right) \log \left(\nu+\frac{1}{2}\right)+\mu^{1 / 2} \int_{h}^{1}(A-a(x))^{1 / 2} d x\right. \\
\left.-\left(\left(\nu+\frac{1}{2}\right) / 2\right) \int_{h}^{1} \theta^{-3 / 2}(x) \theta^{\prime}(x) \log (x-h) d x-\left(\nu+\frac{1}{2}\right) / 2\right\}, & \left.-224 c^{2}+288 d+2520-864\left(\nu+\frac{1}{2}\right) \log \left(\nu+\frac{1}{2}\right)\right], \\
H_{1}(\mu)= & (1 / 1152)\left[144 \nu^{4}+1440 \nu^{3}+\left(5040+32 c^{2}\right) \nu^{2}+\left(5328+864 c^{2}\right) \nu+16 c^{4} / 27\right. \\
J_{1}(\mu)= & 1+(3 / 64)(2 \nu+1) \mu^{-1 / 4} \log \mu-\left(26 \nu+13+12\left(\nu+\frac{1}{2}\right) \log \left(\nu+\frac{1}{2}\right)\right) \mu^{-1 / 4} / 16 .
\end{aligned}
$$

We will now pass to $z_{3}^{\prime}(x, \mu)$. From (3.2.3), and proceeding as in the calculations for $I_{2}(x, \mu)$, we see that $u_{1}^{\prime}(1, \mu)=O\left(\mu^{-1 / 2}\right)$ as $\mu \rightarrow \infty$. Hence, as $\mu \rightarrow \infty$,

$$
\begin{aligned}
& z_{3}^{\prime}\left(h+\mu^{-3 / 16} /(4 b)^{1 / 4}, \mu\right) \\
& =\left(\Gamma(3 / 4) / 2(\pi)^{1 / 2}\right)(4 b)^{1 / 8} F_{1}(\mu) \mu^{1 / 4} \\
& \quad \times\left[1+c \mu^{-1 / 16} / 3+\left(9 \nu^{2}-9 \nu-18+c^{2}\right) \mu^{-1 / 8} / 18\right. \\
& \quad+c\left(27 \nu^{2}+297 \nu+270+c^{2}\right) \mu^{-3 / 16} / 162+(3 / 64)(2 \nu+1) \mu^{-1 / 4} \log \mu \\
& \left.\quad+H_{2}(\mu) \mu^{-1 / 2}+o\left(\mu^{-1 / 4}\right)\right] \\
& \quad \begin{array}{c}
z_{3}^{\prime}(1, \mu)=\left(\Gamma(3 / 4) /(2 \pi)^{1 / 2}\right)(A-a(1))^{1 / 4} G_{1}(\mu) \mu^{1 / 4}\left(J_{1}(\mu)+o\left(\mu^{-1 / 4}\right)\right)
\end{array}
\end{aligned}
$$

where

$$
\begin{array}{r}
H_{2}(\mu)=(1 / 1152)\left[144 \nu^{4}+288 \nu^{3}-\left(1872-32 c^{2}\right) \nu^{2}-\left(7344-736 c^{2}\right) \nu+16 c^{4} / 27\right. \\
\left.+416 c^{2}+288 d-4392-864\left(\nu+\frac{1}{2}\right) \log \left(\nu+\frac{1}{2}\right)\right] .
\end{array}
$$

As a second solution to equation (3.2.1) we consider

$$
\begin{aligned}
& z_{4}(x, \mu)=\left(w^{\prime}(x, \mu)\right)^{-1 / 2} U_{3}\left(\mu^{1 / 4} w(x, \mu)\right)\left(1+u_{2}(x, \mu)\right) \\
& u_{2}(1, \mu)=u_{2}^{\prime}(1, \mu)=0
\end{aligned}
$$

and proceeding precisely as above we have, as $\mu \rightarrow \infty$,

$$
\begin{aligned}
& z_{4}\left(h+\mu^{-3 / 16} /(4 b)^{1 / 4}, \mu\right) \\
& =2(2 / \pi)^{1 / 2}\left(\Gamma(5 / 4) /(4 b)^{1 / 8}\right) F_{1}^{-1}(\mu) \\
& \times\left[1-c \mu^{-1 / 16} / 3-\left(9 \nu^{2}-9 \nu-c^{2}\right) \mu^{-1 / 8} / 18\right. \\
& \left.+c\left(27 \nu^{2}-351 \nu-324-c^{2}\right) \mu^{-3 / 16} / 162+H_{3}(\mu) \mu^{-1 / 4}+o\left(\mu^{-1 / 4}\right)\right], \\
& z_{4}^{\prime}\left(h+\mu^{-3 / 16} /(4 b)^{1 / 4}, \mu\right) \\
& =-(2 / \pi)^{1 / 2}(4 b)^{1 / 8} \Gamma(5 / 4) F_{1}^{-1}(\mu) \mu^{5 / 16} \\
& \times\left[1-c \mu^{-1 / 16} / 3-\left(9 \nu^{2}+27 \nu-c^{2}\right) \mu^{-1 / 8} / 18\right. \\
& \left.+c\left(27 \nu^{2}-243 \nu-c^{2}\right) \mu^{-3 / 16} / 162+H_{4}(\mu) \mu^{-1 / 4}+o\left(\mu^{-1 / 4}\right)\right], \\
& z_{4}(1, \mu)=\left(2 \Gamma(5 / 4) /(\pi)^{1 / 2}\right)(A-a(1))^{-1 / 4} G_{1}^{-1}(\mu)\left(1+o\left(\mu^{-1 / 4}\right)\right), \\
& z_{4}^{\prime}(1, \mu)=-\left(2 \Gamma(5 / 4) /(\pi)^{1 / 2}\right)(A-a(1))^{1 / 4} G_{1}^{-1}(\mu) \mu^{1 / 2}\left(1+o\left(\mu^{-1 / 4}\right)\right) \text {, }
\end{aligned}
$$


where

$$
\begin{gathered}
H_{3}(\mu)=(1 / 1152)\left[144 \nu^{4}-864 \nu^{3}+\left(1584-32 c^{2}\right) \nu^{2}\right. \\
\left.-\left(864-800 c^{2}\right) \nu+16 c^{4} / 27+1056 c^{2}-288 d\right], \\
H_{4}(\mu)=(1 / 1152)\left[144 \nu^{4}+288 \nu^{3}-\left(1872+32 c^{2}\right) \nu^{2}\right. \\
\left.+\left(1440+672 c^{2}\right) \nu+16 c^{4} / 27+288 c^{2}-288 d\right] .
\end{gathered}
$$

If $W$ denotes the Wronskian, we see that $W\left(z_{3}, z_{4}\right)(\mu)=-\mu^{1 / 4}\left(J_{1}(\mu)+o\left(\mu^{-1 / 4}\right)\right)$ as $\mu \rightarrow \infty$; and hence for all sufficiently large values of $\mu, z_{3}(x, \mu)$ and $z_{4}(x, \mu)$ form a fundamental set of solutions for (3.2.1).

3.3. The interval $0 \leqq x \leqq\left(h-\mu^{-3 / 16} /(4 b)^{1 / 4}\right)$. Here we can proceed precisely as in subsection 3.2 and for all sufficiently large values of $\mu$ introduce a fundamental set of solutions, $z_{1}(x, \mu)$ and $z_{2}(x, \mu)$, for the differential equation (1.1.m) in the interval $0 \leqq x \leqq\left(h-\mu^{-3 / 16} /(4 b)^{1 / 4}\right)$. Then with the same notation as in subsection 3.2 , we have, as $\mu \rightarrow \infty$,

$$
\begin{aligned}
& z_{1}\left(h-\mu^{-3 / 16} /(4 b)^{1 / 4}, \mu\right) \\
& =\left(\Gamma\left(\frac{3}{4}\right) /(\pi)^{1 / 2}(4 b)^{1 / 8}\right) F_{1}(\mu) \mu^{-1 / 16} \\
& \times\left[1-c \mu^{-1 / 16} / 3+\left(9 \nu^{2}+27 \nu+18+c^{2}\right) \mu^{-1 / 8} / 18\right. \\
& -c\left(27 \nu^{2}+405 \nu+54+c^{2}\right) \mu^{-3 / 16} / 162+(3 / 64)(2 \nu+1) \mu^{-1 / 4} \log \mu \\
& z_{1}^{\prime}\left(h-\mu^{-3 / 16} /(4 b)^{1 / 4}, \mu\right) \\
& \left.+H_{1}(\mu) \mu^{-1 / 4}+o\left(\mu^{-1 / 4}\right)\right] \\
& =-\left(\Gamma\left(\frac{3}{4}\right) / 2(\pi)^{1 / 2}\right)(4 b)^{1 / 8} F_{1}(\mu) \mu^{1 / 4} \\
& \times\left[1-c \mu^{-1 / 16} / 3+\left(9 v^{2}-9 v-18+c^{2}\right) \mu^{-1 / 8} / 18\right. \\
& -c\left(27 \nu^{2}+297 \nu+270+c^{2}\right) \mu^{-3 / 16} / 162+(3 / 64)(2 \nu+1) \mu^{-1 / 4} \log \mu \\
& \left.+H_{2}(\mu) \mu^{-1 / 4}+o\left(\mu^{-1 / 4}\right)\right] \text {, } \\
& z_{1}(0, \mu)=\left(\Gamma\left(\frac{3}{4}\right) /(2 \pi)^{1 / 2}\right)(A-a(0))^{-1 / 4} G_{2}(\mu) \mu^{-1 / 4}\left(J_{1}(\mu)+o\left(\mu^{-1 / 4}\right)\right) \text {, } \\
& z_{1}^{\prime}(0, \mu)=-\left(\Gamma\left(\frac{3}{4}\right) /(2 \pi)^{1 / 2}\right)(A-a(0))^{1 / 4} G_{2}(\mu) \mu^{1 / 4}\left(J_{1}(\mu)+o\left(\mu^{-1 / 4}\right)\right) \text {, }
\end{aligned}
$$

where

$$
\begin{aligned}
& G_{2}(\mu)=\mu^{-v / 4}\left((4 b)^{1 / 4} \exp \left\{h(b /(A-a(0)))^{1 / 2} \log h\right\}\right)^{-(v+1 / 2)} \\
& \times \exp \left\{\left(\left(\nu+\frac{1}{2}\right) / 2\right) \log \left(\nu+\frac{1}{2}\right)+\mu^{1 / 2} \int_{0}^{h}(A-a(x))^{1 / 2} d x\right. \\
& \left.+\left(\left(\nu+\frac{1}{2}\right) / 2\right) \int_{0}^{h} \theta^{-3 / 2}(x) \theta^{\prime}(x) \log (h-x) d x-\left(\nu+\frac{1}{2}\right) / 2\right\} ; \\
& z_{2}\left(h-\mu^{-3 / 16} /(4 b)^{1 / 4}, \mu\right) \\
& =2(2 / \pi)^{1 / 2}\left(\Gamma\left(\frac{5}{4}\right) /(4 b)^{1 / 8}\right) F_{1}^{-1}(\mu) \\
& \times\left[1+c \mu^{-1 / 16} / 3-\left(9 \nu^{2}-9 \nu-c^{2}\right) \mu^{-1 / 8} / 18\right. \\
& z_{2}^{\prime}\left(h-\mu^{-3 / 16} /(4 b)^{1 / 4}, \mu\right) \\
& \left.-c\left(27 \nu^{2}-351 \nu-324-c^{2}\right) \mu^{-3 / 16} / 162+H_{3}(\mu) \mu^{-1 / 4}+o\left(\mu^{-1 / 4}\right)\right], \\
& =(2 / \pi)^{1 / 2}(4 b)^{1 / 8} \Gamma\left(\frac{5}{4}\right) F_{1}^{-1}(\mu) \mu^{5 / 16} \\
& \times\left[1+c \mu^{-1 / 16} / 3-\left(9 \nu^{2}+27 \nu-c^{2}\right) \mu^{-1 / 8} / 18\right. \\
& \left.-c\left(27 \nu^{2}-243 \nu-c^{2}\right) \mu^{-3 / 16} / 162+H_{4}(\mu) \mu^{-1 / 4}+o\left(\mu^{-1 / 4}\right)\right], \\
& z_{2}(0, \mu)=\left(2 \Gamma\left(\frac{5}{4}\right) /(\pi)^{1 / 2}\right)(A-a(0))^{-1 / 4} G_{2}^{-1}(\mu)\left(1+o\left(\mu^{-1 / 4}\right)\right) \text {, } \\
& z_{2}^{\prime}(0, \mu)=\left(2 \Gamma\left(\frac{5}{4}\right) /(\pi)^{1 / 2}\right)(A-a(0))^{1 / 4} G_{2}^{-1}(\mu) \mu^{1 / 2}\left(1+o\left(\mu^{-1 / 4}\right)\right) \text {. }
\end{aligned}
$$


4. Main results for the case $a(0)<A$ and $a(1)<A$. In this section we consider the case where $a(x)=A$ at precisely the finite set of points $\left\{h_{r}\right\}_{r=1}^{p}$, where $p \geqq 1$ and $0<h_{1}<\cdots<h_{p}<1$. Then throughout this section, unless otherwise stated, we shall consider the integer $m$ fixed as before and use the results of $\S 3$ to verify (1.3). We shall see in the sequel the important role played in the analysis by the functions $\nu_{r}=\nu_{r}(\mu), r=1, \ldots, p$ (see $\left.\S 2\right)$. Finally, throughout this section, as well as in $\S \S 5$ and 6 , the terminology of $\S 2$ will be used; and also in the sequel when we refer to the set $R$ we shall mean the subset of the rationals defined in Appendix III.

THEOREM 4.1. If $p=1$, then (1.3) is valid with $A_{1, m}=G_{1,1}(1, m), A_{2, m}=0$, and $A_{3, m}=G_{1,2}(1, m)$.

Proof. We will write $h$ for $h_{1}, b$ for $b_{1}, c$ for $c_{1}, d$ for $d_{1}, \nu$ for $\nu_{1}$, and apply directly the results of $\S 3$. Then for $\mu \geqq \mu^{*}$ (see $\S 3$ ), put

$$
\begin{aligned}
y_{L}(x, \mu)=[ & \left.-\cos \alpha z_{2}(0, \mu)+\sin \alpha z_{2}^{\prime}(0, \mu)\right] z_{1}(x, \mu) \\
& +\left[\cos \alpha z_{1}(0, \mu)-\sin \alpha z_{1}^{\prime}(0, \mu)\right] z_{2}(x, \mu), \\
y_{R}(x, \mu)=[ & \left.-\cos \beta z_{4}(1, \mu)+\sin \beta z_{4}^{\prime}(1, \mu)\right] z_{3}(x, \mu) \\
& +\left[\cos \beta z_{3}(1, \mu)-\sin \beta z_{3}^{\prime}(1, \mu)\right] z_{4}(x, \mu) .
\end{aligned}
$$

Hence for an eigenfunction we must have, for all sufficiently large values of $\mu$,

$$
\left|\begin{array}{cccc}
y_{L}\left(h^{-}, \mu\right) & y_{1}\left(h^{-}, \mu\right) & y_{2}\left(h^{-}, \mu\right) & 0 \\
y_{L}^{\prime}\left(h^{-}, \mu\right) & y_{1}^{\prime}\left(h^{-}, \mu\right) & y_{2}^{\prime}\left(h^{-}, \mu\right) & 0 \\
0 & y_{1}\left(h^{+}, \mu\right) & y_{2}\left(h^{+}, \mu\right) & y_{R}\left(h^{+}, \mu\right) \\
0 & y_{1}^{\prime}\left(h^{+}, \mu\right) & y_{2}^{\prime}\left(h^{+}, \mu\right) & y_{R}^{\prime}\left(h^{+}, \mu\right)
\end{array}\right|=0
$$

where $h^{-}=h-\mu^{-3 / 16} /(4 b)^{1 / 4}, h^{+}=h+\mu^{-3 / 16} /(4 b)^{1 / 4}$. If we refer to the formulae of subsections 3.1, 3.2, and 3.3 and put $W(x)=2 c^{2} C_{2}(x)-2 d B_{2}(x)+q(h) E_{2}(x) /(b)^{1 / 2}$, then this gives

$$
\sin \pi(\nu+1)-\mu^{-1 / 2}(W(\nu)+f(\mu)) \cos \pi(\nu+1)=0,
$$

where $f(\mu)=o(1)$ as $\mu \rightarrow \infty$. Hence we see that as $\mu \rightarrow \infty, \nu$ must tend to an integer, which is nonnegative from our condition on $\nu$, and this integer must be $m([4, \mathrm{p} .136]$, [15, Theorems 2.1 and 2.2]). Putting $\nu(\mu)=m+\varepsilon(\mu)$, we have for all $\mu$ sufficiently large, $\tan \pi \varepsilon(\mu)=\mu^{-1 / 2}(W(m+\varepsilon(\mu))+f(\mu))$. For $\mu>0$ put $w(\varepsilon, \mu)=\tan \pi \varepsilon-$ $\mu^{-1 / 2} W(m+\varepsilon)$; we observe that (1) for $\mu$ fixed, $w(\varepsilon, \mu)$ is analytic in $|\varepsilon| \leqq \frac{1}{4}$ and $\{w(\varepsilon, \mu)-w(0, \mu)\}$ has precisely one zero in this disc if $\mu$ is sufficiently large, (2) $\partial w(0, \mu) / \partial \varepsilon=\pi+O\left(\mu^{-1 / 2}\right)$ as $\mu \rightarrow \infty$, and (3) on the circle $|\varepsilon|=\pi / 256,|w(\varepsilon, \mu)-w(0, \mu)|$ $>\pi^{2} / 768$ for all $\mu$ sufficiently large. Hence for all sufficiently large values of $\mu$ we may apply the inverse function theorem, and since bounds for the coefficients in the expansion

$$
\varepsilon(\mu)=\sum_{n=1}^{\infty} g_{n}(\mu)\left(\mu^{-1 / 2} f(\mu)-w(0, \mu)\right)^{n}
$$


may be obtained independently of $\mu$, we see that, as $\mu \rightarrow \infty, \pi \varepsilon(\mu)=\mu^{-1 / 2} W(m)$ $+o\left(\mu^{-1 / 2}\right)$, and our theorem follows from Appendix I and $\S 2$.

From now on in this section we shall always assume that $p>1$. Then we observe from $\S 2$ that $\nu_{r}$ is bounded and greater than $-\frac{1}{2}$ for $\mu \geqq \mu_{m}, r=1, \ldots, p$, and, for $\mu>0$,

$$
\left(b_{1}\right)^{1 / 2}\left(\nu_{1}+\frac{1}{2}\right)=\left(b_{r}\right)^{1 / 2}\left(\nu_{r}+\frac{1}{2}\right), \quad r=1, \ldots, p .
$$

TheOREM 4.2. As $\mu \rightarrow \infty, \nu_{r}$ tends to a finite limit, $r=1, \ldots, p$, and at least one such limit is an integer.

Proof. For $r=1, \ldots, p$ the results of $\S 3$ may be modified in an obvious way so as to apply to the intervals $\left(h_{r-1}+h_{r}\right) / 2 \leqq x \leqq\left(h_{r}-H_{r}(\mu)\right),\left(h_{r}-H_{r}(\mu)\right) \leqq x \leqq$ $\left(h_{r}+H_{r}(\mu)\right),\left(h_{r}+H_{r}(\mu)\right) \leqq x \leqq\left(h_{r}+h_{r+1}\right) / 2$, where $H_{r}(\mu)=\mu^{-3 / 16} /\left(4 b_{r}\right)^{1 / 4}, h_{0}=-h_{1}$, and $h_{p+1}=2-h_{p}$. Then proceeding as in Theorem 4.1, but now matching our solutions in value and in value of the first derivative at the points $\left(h_{r} \pm H_{r}(\mu)\right)$, $r=1, \ldots, p,\left(h_{r+1}+h_{r}\right) / 2, r=1, \ldots,(p-1)$, we obtain

$$
\prod_{r=1}^{p} K_{r}(\mu)=o\left(\exp \left\{-\Delta \mu^{1 / 2}\right\}\right) \quad \text { as } \mu \rightarrow \infty,
$$

where

$$
\begin{aligned}
K_{r}(\mu) & =\left[\sin \pi\left(\nu_{r}+1\right)-\mu^{-1 / 2}\left(W_{r}\left(\nu_{r}\right)+f_{r}(\mu)\right) \cos \pi\left(\nu_{r}+1\right)\right], \\
f_{r}(\mu) & =o(1) \text { as } \mu \rightarrow \infty, \\
W_{r}(x) & =2 c_{r}^{2} C_{2}(x)-2 d_{r} B_{2}(x)+q\left(h_{r}\right) E_{2}(x) /\left(b_{r}\right)^{1 / 2}, \quad r=1, \ldots, p,
\end{aligned}
$$

$C_{2}(x), B_{2}(x), E_{2}(x)$ as defined in Appendix $\mathrm{I}$, and $\Delta$ is a positive constant.

Now assume that our theorem is false; then with $\lim _{\inf _{\mu \rightarrow \infty}} \nu_{1}=k_{1}, \lim \sup _{\mu \rightarrow \infty} \nu_{1}$ $=k_{2}$, we have $k_{1}<k_{2}$. Choose $\varepsilon$ so that $0<\varepsilon<\left(k_{2}-k_{1}\right) / 8$ and denote by $X_{1}$ (possibly empty) the set of integers lying in $\left[k_{1}-\varepsilon, k_{2}+\varepsilon\right]$, and by $X_{r}$ (possibly empty) the set of points $x$ lying in $\left[k_{1}-\varepsilon, k_{2}+\varepsilon\right]$ for which $\left\{\left(b_{1} / b_{r}\right)^{1 / 2}\left(x+\frac{1}{2}\right)-\frac{1}{2}\right\}$ is an integer, $r=2, \ldots, p$. Since $\bigcup_{r=1}^{p} X_{r}$ is at most a finite set, there is an $x_{0}$ such that $x_{0} \in\left[k_{1}+\varepsilon, k_{2}-\varepsilon\right]-\bigcup_{r=1}^{p} X_{r}$. Thus, since $\nu_{1}(\mu)$ is a continuous function of $\mu$ for $\mu>0$, there is a sequence of values of $\mu,\left\{\mu_{i}\right\}_{i=1}^{\infty}, 0<\mu_{1}<\mu_{2}<\cdots, \lim _{i \rightarrow \infty} \cdot \mu_{i}=\infty$, such that $\nu_{1}\left(\mu_{i}\right)=x_{0}, i=1,2, \ldots$, and therefore $\prod_{r=1}^{p} \sin \pi\left(\nu_{r}\left(\mu_{i}\right)+1\right)=k, i=1$, $2, \ldots$, where $k$ is a nonzero constant; but this is incompatible with (4.3), and hence $\lim _{\mu \rightarrow \infty} \nu_{r}$ exists and is finite, $r=1, \ldots, p$, and clearly (4.3) implies that at least one such limit is an integer. This proves the theorem.

From now on we shall denote $\lim _{\mu \rightarrow \infty} \nu_{r}$ by $\nu_{r}(\infty), r=1, \ldots, p$; and we observe from $\S 2$ that if $\nu_{r}(\infty)$ is an integer, then it must be a nonnegative integer.

THEOREM 4.3. If $\left(b_{i} / b_{j}\right) \notin R$ for $i, j=1, \ldots, p$ and $i \neq j$, then the elements of the set $\left\{\nu_{r}(\infty)\right\}_{r=1}^{p}$ can be determined. Moreover, precisely one element of this set is an integer, and if we denote this element by $\nu_{r^{*}}(\infty)$ and put $\nu_{r^{*}}(\infty)=n^{*}$, then (1.3) is valid with $A_{1, m}=G_{1,1}\left(r^{*}, n^{*}\right), A_{2, m}=0$, and $A_{3, m}=G_{1,2}\left(r^{*}, n^{*}\right)$. 
Proof. From (4.2) we see that

$$
\left(b_{1}\right)^{1 / 2}\left(\nu_{1}(\infty)+\frac{1}{2}\right)=\left(b_{r}\right)^{1 / 2}\left(\nu_{r}(\infty)+\frac{1}{2}\right), \quad r=1, \ldots, p,
$$

and hence the conditions of our theorem imply that precisely one element of the set $\left\{\nu_{r}(\infty)\right\}_{r=1}^{p}$ is an integer. We shall, as stated above, denote this element by $\nu_{r^{*}}(\infty)$ and put $\nu_{r^{*}}(\infty)=n^{*}$.

We now assert that in order to determine the tuple $\left(r^{*}, n^{*}\right)$, we need only put $a_{r}=b_{r}, r=1, \ldots, p$, in subsection III. 1 of Appendix III, and with $f_{r}(n)$ given by equation (III.1.1), solve for $\left(r^{\dagger}, n^{\dagger}\right)$ the equation $f_{r}(n)=m$ (see Theorem III.1.1); and then we must have $r^{*}=r^{\dagger}, n^{*}=n^{\dagger}$. With $\left(r^{*}, n^{*}\right)$ determined we can then use (4.5) to determine $\nu_{r}(\infty), r=1, \ldots, p$. Since $\nu_{r^{*}}(\infty)$ is the only element of the set $\left\{\nu_{r}(\infty)\right\}_{r=1}^{p}$ which is an integer, we can now argue with (4.3) in precisely the same way as we did with (4.1) to verify the last statement of our theorem.

We shall now prove the above assertion. First, we observe from (4.2), (4.3), and (4.5) that $\left(\nu_{r}-\nu_{r}(\infty)\right)=O\left(\mu^{-1 / 2}\right)$ as $\mu \rightarrow \infty, r=1, \ldots, p$. Then for $r=1, \ldots, p$, and with $h_{0}=-h_{1}, h_{p+1}=2-h_{p}$, put $J_{r}=\left\{x \mid\left(h_{r-1}+h_{r}\right) / 2 \leqq x \leqq\left(h_{r}+h_{r+1}\right) / 2\right\}, \quad \theta_{r}(x)$ $=\left((A-a(x)) /\left(x-h_{r}\right)^{2} b_{r}\right)$ for $x \in J_{r}-\left\{h_{r}\right\}, \quad \theta_{r}\left(h_{r}\right)=1$, and $\theta_{r}=\inf _{x \in J_{r}} \theta_{r}(x)$. Let $\theta=\min _{1 \leqq r \leqq p} \theta_{r}, b=\min _{1 \leqq r \leqq p} b_{r}$, and $Q=\sup _{0 \leqq x \leqq 1}|q(x)|$; then for the remainder of this proof we shall assume that $\mu \geqq \mu^{\dagger}=\max \left\{\mu_{m},\left(\left(\Lambda_{m}+Q\right) / b h^{2} \theta\right)^{2}\right\}$, where $h=\min _{0 \leqq r \leqq p}\left(h_{r+1}-h_{r}\right) / 8, h_{0}=0, h_{p+1}=1$ and all other terms are defined in $\S 2$. Hence if $\delta(\mu)=\mu^{-1 / 4}\left(\left(\Lambda_{m}+Q\right) / b \theta\right)^{1 / 2}$ and $I_{r}(\mu)=\left\{x|| x-h_{r} \mid \leqq \delta(\mu)\right\}, r=1, \ldots, p$, then $\lambda_{m}(\mu)+\mu a(x)+q(x)<0$ in $[0,1]-\bigcup_{r=1}^{p} I_{r}(\mu)$.

For $\mu \geqq \mu^{\dagger}$ and $1 \leqq r \leqq p$ consider now the mapping of $J_{r}$ into the $s$-axis c'efined by

$$
s=\left(4 \mu b_{r}\right)^{1 / 4}\left(x-h_{r}\right), \quad x \in J_{r}
$$

and denote by $J_{r}(\mu)$ and $I_{r}$ the image on the $s$-axis of $J_{r}$ and $I_{r}(\mu)$, respectively. We note that $I_{r}$ is just the interval $|s| \leqq \delta_{r}$, where $\delta_{r}=\left(4 b_{r}\right)^{1 / 4}\left(\left(\Lambda_{m}+Q\right) / b \theta\right)^{1 / 2}$. Let $y(x, \mu)$ be the eigenfunction of (1.1-2) corresponding to $\lambda_{m}(\mu)$ and satisfying $y(0, \mu)=\sin \alpha, y^{\prime}(0, \mu)=\cos \alpha$, where ${ }^{\prime}=d / d x$; and for $-\infty<s<\infty$, we introduce the functions

$$
\begin{aligned}
V_{1, r}(s) & =D_{v_{r}(\infty)}(s), \\
V_{2, r}(s) & =D_{v_{r}(\infty)}(-s) \text { if } r \neq r^{*}, \\
V_{2, r^{*}}(s) & =(1 / 2 i)\left[\exp \left(i \pi n^{*} / 2\right) U_{1}\left(s, n^{*}\right)-\exp \left(-i \pi n^{*} / 2\right) U_{2}\left(s, n^{*}\right)\right],
\end{aligned}
$$

and where all terms are defined in Appendix I. Let $k_{r}=W\left(V_{1, r}, V_{2, r}\right)(s)$, where $W$ denotes the Wronskian; then we observe from [13, pp. 347-348] that $k_{r}=$ $-(2 / \pi)^{1 / 2} \Gamma\left(1+\nu_{r}(\infty)\right) \sin \pi \nu_{r}(\infty)$ if $r \neq r^{*}$, and $k_{r^{*}}=-1$. Hence if

$$
\begin{aligned}
& k_{1, r}^{*}(\mu)=\left(k_{r}\right)^{-1}\left|\begin{array}{cc}
y\left(h_{r}, \mu\right) & V_{2, r}(0) \\
\left(4 \mu b_{r}\right)^{-1 / 4} y^{\prime}\left(h_{r}, \mu\right) & V_{2, r}^{\prime}(0)
\end{array}\right|, \\
& k_{2, r}^{*}(\mu)=\left(k_{r}\right)^{-1}\left|\begin{array}{cc}
V_{1, r}(0) & y\left(h_{r}, \mu\right) \\
V_{1, r}^{\prime}(0) & \left(4 \mu b_{r}\right)^{-1 / 4} y^{\prime}\left(h_{r}, \mu\right)
\end{array}\right|,
\end{aligned}
$$


where $V_{j, r}^{\prime}(0)=d V_{j, r}(0) / d s, j=1,2$, then $\left(k_{1, r}^{*}(\mu)\right)^{2}+\left(k_{2, r}^{*}(\mu)\right)^{2}>0$. Finally, let $\Delta_{r}(\mu)= \pm\left(\left(k_{1, r}^{*}(\mu)\right)^{2}+\left(k_{2, r}^{*}(\mu)\right)^{2}\right)^{-1 / 2}$, where, with $k_{1, r}(\mu)=\Delta_{r}(\mu) k_{1, r}^{*}(\mu)$ and $k_{2, r}(\mu)$ $=\Delta_{r}(\mu) k_{2, r}^{*}(\mu)$, the sign of $\Delta_{r}(\mu)$ is chosen so that (1) if $r^{*}>1$ and $1 \leqq r<r^{*}$, then either $k_{2, r}(\mu)>0$ or $k_{1, r}(\mu)=1$, (2) either $k_{1, r^{*}}(\mu)>0$ or $k_{2, r^{*}}(\mu)=1$, and (3) if $r^{*}<p$ and $r^{*}<r \leqq p$, then either $k_{1, r}(\mu)>0$ or $k_{2, r}(\mu)=1$.

Put $v_{r}(s, \mu)=\Delta_{r}(\mu) y(x, \mu), x \in J_{r}$; then

$$
v_{r}^{\prime \prime}(s, \mu)+\left(v_{r}(\infty)+\frac{1}{2}-s^{2} / 4\right) v_{r}(s, \mu)=F_{r}(s, \mu) v_{r}(s, \mu), \quad s \in J_{r}(\mu), \quad{ }^{\prime}=d / d s,
$$
where $F_{r}(s, \mu)=-\left(q_{r}(s) / 2\left(b_{r}\right)^{1 / 2}\right) \mu^{-1 / 2}+s^{2}\left(\phi_{r}(s)-1\right) / 4+\left(\nu_{r}(\infty)-\nu_{r}\right), q_{r}(s)=q(x)$ and $\phi_{r}(s)=\theta_{r}(x)$ for $x \in J_{r}$, and from the above remarks and the introduction to $\S 3$ we also know that, as $\mu \rightarrow \infty, F_{r}(s, \mu)=O\left(\mu^{-1 / 4}\right)$, uniformly in any bounded subset of the $s$-axis. Hence from (4.7) we see that

$$
v_{r}(s, \mu)=k_{1, r}(\mu) V_{1, r}(s)+k_{2, r}(\mu) V_{2, r}(s)+\int_{0}^{s} K_{r}(s, t) F_{r}(t, \mu) v_{r}(t, \mu) d t,
$$

$s \in J_{r}(\mu)$,

where $K_{r}(s, t)=\left(k_{r}\right)^{-1}\left[V_{1, r}(t) V_{2, r}(s)-V_{1, r}(s) V_{2, r}(t)\right]$, and all other terms are defined above. We note from our previous remarks that $\left(k_{1, r}(\mu)\right)^{2}+\left(k_{2, r}(\mu)\right)^{2}=1$ and (1) if $r^{*}>1$ and $1 \leqq r<r^{*}$ then $k_{2, r}(\mu) \geqq 0$, (2) $k_{1, r^{*}}(\mu) \geqq 0$, and (3) if $r^{*}<p$ and $r^{*}<r \leqq p$ then $k_{1, r}(\mu) \geqq 0$. Also if $S$ is any bounded subset of the $s$-axis and if $S \subset J_{r}(\mu)$ for $\mu \geqq \mu^{*}$, then an application of the Gronwall lemma to (4.8) shows that $\left|v_{r}(s, \mu)\right|$ is uniformly bounded for $s \in S$ and $\mu \geqq \mu^{*}$.

We now assert that $\lim _{\mu \rightarrow \infty} k_{2, r^{*}}(\mu)=0$. For if this is not the case, then from (4.8), the above remarks, and the properties of the parabolic cylinder functions, we see that there is an $s^{*}, s^{*} \notin I_{r^{*}}$, and a sequence of values of $\mu,\left\{\mu_{l}\right\}_{l=1}^{\infty}$, where $\mu^{\dagger}<\mu_{1}<\mu_{2}<\cdots, \lim _{l \rightarrow \infty} \mu_{l}=\infty$, such that $\left|v_{r^{*}}\left(s^{*}, \mu_{l}\right)\right|>2 \sup _{s \in I_{r^{*}}}\left|v_{r^{*}}\left(s, \mu_{l}\right)\right|$ for all $l$ sufficiently large. But, on the other hand, we know [15, Theorems 2.1 and 2.2] that for all $\mu$ sufficiently large the absolute maximum of $\left|v_{r^{*}}(s, \mu)\right|$ in $J_{r^{*}}(\mu)$ is attained in $I_{r^{*}}$.

We also assert that if $r^{*}>1$, then $\lim _{\mu \rightarrow \infty} k_{1, r}(\mu)=0$ for $r=1, \ldots,\left(r^{*}-1\right)$. For $r=1$ the argument follows as above if we make use of the fact [15, Theorem 2.1] that for all sufficiently large values of $\mu$, the absolute maximum of $\left|v_{1}(s, \mu)\right|$ in the interval $\left\{s \mid s \leqq 0, s \in J_{1}(\mu)\right\}$ is attained in $I_{1}$. Now we can choose $s_{1}>0, s_{1} \notin I_{1}$ so that all the real zeros (if any) of $D_{v_{1}(\infty)}(-s)$ are less than $s_{1}$ and $v_{1}\left(s_{1}, \mu\right) v_{1}^{\prime}\left(s_{1}, \mu\right)>0$ for all $\mu$ sufficiently large (this follows from [13, pp. 347-349] and (4.8)). Hence if for large $\mu$ we denote by $x_{1}(\mu)$ the image of $s_{1}$ under the transformation (4.6) (with $r=1$ ) then $y(x, \mu) y^{\prime}(x, \mu)$ is positive in the interval $\left[x_{1}(\mu), h_{2}-\delta(\mu)\right]$ for all $\mu$ sufficiently large ([17, p. 110]); so if $2<r^{*}$, then the same argument as before shows that $\lim _{\mu \rightarrow \infty} k_{1,2}(\mu)=0$. If $2 \leqq j<r^{*}$ and $\lim _{\mu \rightarrow \infty} k_{1, r}(\mu)=0, r=1, \ldots, j$, then we can choose $s_{j}>0, s_{j} \notin I_{j}$, so that all the real zeros (if any) of $D_{v_{j}(\infty)}(-s)$ are less than $s_{j}$ and $v_{j}\left(s_{j}, \mu\right) v_{j}^{\prime}\left(s_{j}, \mu\right)>0$ for all $\mu$ sufficiently large. Hence if for large $\mu$ we denote by $x_{j}(\mu)$ the image of $s_{j}$ under the transformation (4.6) (with $r=j$ ) then $y(x, \mu) y^{\prime}(x, \mu)$ is positive in the interval $\left[x_{j}(\mu), h_{j+1}-\delta(\mu)\right]$ for all $\mu$ sufficiently large; so if $(j+1)$ 
$<r^{*}$, then the above argument also shows that $\lim _{\mu \rightarrow \infty} k_{1, j+1}(\mu)=0$. Our assertion now follows by induction.

In a similar manner we can show that if $r^{*}<p$, then $\lim _{\mu \rightarrow \infty} k_{2, r}(\mu)=0$ for $r=\left(r^{*}+1\right), \ldots, p$. Here for $r=\left(r^{*}+1\right), \ldots, p$, we choose $s_{r}<0, s_{r} \notin I_{r}$, so that all the real zeros (if any) of $D_{v_{r}(\infty)}(s)$ are greater than $s_{r}$ and $v_{r}\left(s_{r}, \mu\right) v_{r}^{\prime}\left(s_{r}, \mu\right)<0$ for all $\mu$ sufficiently large. Hence if for large $\mu$ we denote by $x_{r}(\mu)$ the image of $s_{r}$ under the transformation (4.6), then $y(x, \mu) y^{\prime}(x, \mu)$ is negative in the interval $\left[h_{r-1}+\delta(\mu)\right.$, $x_{r}(\mu)$ ] for all $\mu$ sufficiently large.

Now we first note from [15, Theorem 2.1] that $y(x, \mu)$ has no zeros in the intervals $0<x \leqq h_{1}-\delta(\mu)$ and $h_{p}+\delta(\mu) \leqq x<1$ for all $\mu$ sufficiently large. Then for $r \neq r^{*}$ denote by $N_{r}$ the number of real zeros of $D_{v_{r}(\infty)}(s)$; and for each $\mu \geqq \mu^{\dagger}$ denote also by $J_{1}^{*}(\mu)$ the interval obtained by deleting from $J_{1}(\mu)$ its left-hand end point, by $J_{p}^{*}(\mu)$ the interval obtained by deleting from $J_{p}(\mu)$ its right-hand end point, and if $p>2$ put $J_{r}^{*}(\mu)=J_{r}(\mu)$ for $r=2, \ldots,(p-1)$. Hence if $r^{*}>1$ and $1 \leqq r<r^{*}$, then the above arguments show that for all $\mu$ sufficiently large $v_{r}(s, \mu)$ has precisely $N_{r}$ zeros in $J_{r}^{*}(\mu)$ and all these zeros lie in the interval $\left[-\delta_{r}, s_{r}\right]$; here we have used the fact, which follows directly from (4.8) and our above remarks, that in any bounded subset of the $s$-axis $v_{r}(s, \mu)$ and $v_{r}^{\prime}(s, \mu)$ tend uniformly to $V_{2, r}(s)$ and $V_{2, r}^{\prime}(s)$, respectively, as $\mu \rightarrow \infty$. Similarly if $r^{*}<p$ and $r^{*}<r \leqq p$, then for all $\mu$ sufficiently large $v_{r}(s, \mu)$ has precisely $N_{r}$ zeros in $J_{r}^{*}(\mu)$ and all these zeros lie in the interval $\left[s_{r}, \delta_{r}\right]$. Also for all $\mu$ sufficiently large $v_{r^{*}}(s, \mu)$ has precisely $n^{*}$ zeros in $J_{r^{*}}^{*}(\mu)$ and all these zeros lie in the interval $I_{r^{*}}$. But then we must have $n^{*}+\sum_{r=1 ; r \neq r^{*}}^{p} N_{r}=m$, or from [14, p. 126], $n^{*}+\sum_{r=1 ; r \neq r^{*}}^{p}\left[\nu_{r}(\infty)+1\right]^{*}=m$, where $[x]^{*}$ is the greatest positive integer less than $x$ or zero if such a positive integer does not exist. Finally, from (4.5) we see that

$$
n^{*}+\sum_{r=1 ; r \neq r^{*}}^{p}\left[\left(b_{r^{*}} / b_{r}\right)^{1 / 2}\left(n^{*}+\frac{1}{2}\right)+\frac{1}{2}\right]^{*}=m
$$

Now referring to subsection III. 1 of Appendix III, and putting $a_{r}=b_{r}, r=1, \ldots, p$, we then see from (4.9) that $\left(r^{*}, n^{*}\right)$ renders soluble the equation $f_{r}(n)=m$. But Theorem III.1.1 states that this equation has a unique solution, namely $\left(r^{\dagger}, n^{\dagger}\right)$; hence $r^{*}=r^{\dagger}, n^{*}=n^{\dagger}$, and this completes the proof of our theorem.

THEOREM 4.4. If $\left(b_{i} / b_{j}\right) \in R$ for at least one pair of integers $(i, j)$, where $1 \leqq i, j \leqq p$ and $i \neq j$, then the elements of the set $\left\{\nu_{r}(\infty)\right\}_{r=1}^{p}$ can be determined.

Proof. Let (1.1) be replaced by the perturbed equation

$$
y^{\prime \prime}(x)+(\lambda+\mu a(x, t)+q(x)) y(x)=0, \quad 0 \leqq x \leqq 1, \quad t>0,
$$

where $a(x, t)=a(x)-\operatorname{tg}(x)$,

$$
\begin{aligned}
g(x)= & \left(\left(h_{1}+h_{2}\right) / 2-x\right)^{5}\left(x-h_{1}\right)^{2} g_{1}, \quad 0 \leqq x \leqq\left(h_{1}+h_{2}\right) / 2, \\
= & \left(x-\left(h_{r}+h_{r-1}\right) / 2\right)^{5}\left(\left(h_{r+1}+h_{r}\right) / 2-x\right)^{5}\left(x-h_{r}\right)^{2} g_{r}, \\
& \left(h_{r}+h_{r-1}\right) / 2 \leqq x \leqq\left(h_{r+1}+h_{r}\right) / 2, \quad r=2, \ldots,(p-1), \\
= & \left(x-\left(h_{p}+h_{p-1}\right) / 2\right)^{5}\left(x-h_{p}\right)^{2} g_{p}, \quad\left(h_{p}+h_{p-1}\right) / 2 \leqq x \leqq 1,
\end{aligned}
$$


and with $g_{1}^{*}=\left(\left(h_{2}-h_{1}\right) / 2\right)^{5} g_{1}, g_{r}^{*}=\left(\left(h_{r+1}-h_{r}\right) / 2\right)^{5}\left(\left(h_{r}-h_{r-1}\right) / 2\right)^{5} g_{r}, r=2, \ldots,(p-1)$, $g_{p}^{*}=\left(\left(h_{p}-h_{p-1}\right) / 2\right)^{5} g_{p}$, the positive constants $g_{r}^{*}, r=1, \ldots, p$, are chosen in the following way. If all the $b_{r}, r=1, \ldots, p$, are equal, we choose the $g_{r}^{*}$ so that $g_{1}^{*}>g_{2}^{*}>\cdots>g_{p}^{*}>0$. If $b_{r(1,1)}=b_{r(1,2)}=\cdots=b_{r\left(1, j_{1}\right)}<b_{r(2,1)}=b_{r(2,2)}=\cdots=b_{r\left(2, j_{2}\right)}$ $<\cdots<b_{r(l, 1)}=b_{r(l, 2)}=\cdots=b_{r\left(l, j_{l}\right)}$, where $l>1, j_{i} \geqq 1$ for $i=1, \ldots, l, \sum_{i=1}^{l} j_{i}=p$, and $h_{r(i, 1)}<h_{r(i, 2)}<\cdots<h_{r\left(i, j_{i}\right)}$ if $j_{i}>1$ for $i=1, \ldots, l$, then the positive constants $g_{r}^{*}$, $r=1, \ldots, p$, are chosen so that $g_{r(1,1)}^{*}>g_{r(1,2)}^{*}>\cdots>g_{r\left(1, j_{1}\right)}^{*}$, and for $i=2, \ldots, l$, $g_{r\left(i-1, j_{i-1}\right)}^{*}>g_{r(i, 1)}^{*}>g_{r(i, 2)}^{*}>\cdots>g_{r\left(i, j_{i}\right)}^{*}$.

Putting $b_{r}(t)=-\left(\frac{1}{2}\right) d^{2} a\left(h_{r}, t\right) / d x^{2}=b_{r}+t g_{r}^{*}, r=1, \ldots, p$, it is now a simple matter to verify that if $1 \leqq i, j \leqq p$ and $i \neq j$, then $\left(b_{i}(t) / b_{j}(t)\right)$ is continuous, positive, and either strictly increasing or strictly decreasing for $t \geqq 0$.

Let $R_{m}^{*}=\left\{x \mid x=((2 j+1) /(2 k+1))^{2}\right.$, where $j$ and $k$ are integers, $j=0, \ldots, k$ and $k=0, \ldots,(m+2)\}$. If $1 \leqq i, j \leqq p, i \neq j$, and (1) $\left(b_{i} / b_{j}\right) \notin R_{m}^{*}$, then denote by $d_{i, j}$ the minimum distance between $\left(b_{i} / b_{j}\right)$ and the points of $R_{m}^{*}$, and choose the positive number $t_{i, j}$ so that $\left|\left(b_{i} / b_{j}\right)-\left(b_{i}(t) / b_{j}(t)\right)\right|<d_{i, j} / 2$ for $0 \leqq t \leqq t_{i, j} ;(2)\left(b_{i} / b_{j}\right) \in R_{m}^{*}$, then denote by $d$ the minimum distance between the points of $R_{m}^{*}$, and choose the positive number $t_{i, j}$ so that $\left|\left(b_{i} / b_{j}\right)-\left(b_{i}(t) / b_{j}(t)\right)\right|<d / 2$ for $0 \leqq t \leqq t_{i, j}$. Hence putting

$$
t_{1}=\min _{1 \leqq i, j \leqq p ; i \neq j}\left\{t_{i, j}\right\}
$$

we conclude that if $1 \leqq i, j \leqq p$ and $i \neq j$, then $\left(b_{i}(t) / b_{j}(t)\right) \notin R_{m}^{*}$ for $0<t \leqq t_{1}$.

Let $\lambda_{m}(\mu, t)$ be the $m$ th eigenvalue of system (4.10), (1.2), and for $\mu>0$ put $\Lambda_{m}(\mu, t)=\left(\left(\lambda_{m}(\mu, t)+\mu A\right) / \mu^{1 / 2}\right), \nu_{r}(\mu, t)=\left\{\left(\Lambda_{m}(\mu, t) / 2\left(b_{r}(t)\right)^{1 / 2}\right)-\frac{1}{2}\right\}, r=1, \ldots, p$. Then it is clear that we may argue with $\lambda_{m}(\mu, t)$ in precisely the same way as we did with $\lambda_{m}(\mu)$, and indeed by modifying (4.3) and Theorem 4.2 so as to apply to system (4.10), (1.2), we then know that $\nu_{r}(\mu, t)$ tends to a finite limit as $\mu \rightarrow \infty, r=1, \ldots, p$, and at least one such limit is an integer. We shall denote $\lim _{\mu \rightarrow \infty} \nu_{r}(\mu, t)$ by $\nu_{r}(\infty, t)$, $r=1, \ldots, p$.

We remark now that if $0<t \leqq t_{1}$, then precisely one element of the set $\left\{\nu_{r}(\infty, t)\right\}_{r=1}^{p}$ is an integer; and we shall denote this element by $\nu_{r^{\dagger}(t)}(\infty, t)$ and put $\nu_{r^{\dagger}(t)}(\infty, t)$ $=n^{\dagger}(t)$. To see this, we return to the proof of Theorem 4.3, modify (4.6) and the results given in the paragraph preceding this equation so as now to apply to system (4.10), (1.2) and $\lambda_{m}(\mu, t)$, and let the eigenfunction $y(x, \mu)$ now correspond to $\lambda_{m}(\mu, t)$. Putting $v_{r}(s, \mu)=y(x, \mu), x \in J_{r}, r=1, \ldots, p$, we consider the analogue of (4.7) and argue with Sturm's fundamental theorem to show that $\nu_{r}(\infty, t) \leqq m+2$, $r=1, \ldots, p$. Hence if $i \neq j$, and $\nu_{i}(\infty, t)=n_{i}, \nu_{j}(\infty, t)=n_{j}$, where $n_{i}$ and $n_{j}$ are integers, then (see $(4.5))\left(b_{i}(t)\right)^{1 / 2}\left(n_{i}+\frac{1}{2}\right)=\left(b_{j}(t)\right)^{1 / 2}\left(n_{j}+\frac{1}{2}\right)$ and either $\left(b_{i}(t) / b_{j}(t)\right)$ or $\left(b_{j}(t) / b_{i}(t)\right)$ is a member of $R_{m}^{*}$, which is impossible.

We now assert that if $0<t \leqq t_{1}$, then the tuple $\left(r^{\dagger}(t), n^{\dagger}(t)\right)$ is cetermined by putting $a_{r}=b_{r}(t), r=1, \ldots, p$, in subsection III.1 of Appendix III, and with $f_{r}(n)$ given by equation (III.1.1), solving for $\left(r^{\dagger}, n^{\dagger}\right)$ the equation $f_{r}(n)=m$ (see Theorem III.1.2) then we must have $r^{\dagger}(t)=r^{\dagger}, n^{\dagger}(t)=n^{\dagger}$. To see this, we make use of the fact 
that $\nu_{r^{\dagger}(t)}(\infty, t)$ is the only element of the set $\left\{\nu_{r}(\infty, t)\right\}_{r=1}^{p}$ which is an integer and hence we may argue precisely as in the proof of Theorem 4.3 to show that

$$
n^{\dagger}(t)+\sum_{r=1 ; r \neq r \dagger(t)}^{p}\left[\left(b_{r^{\dagger}(t)}(t) / b_{r}(t)\right)^{1 / 2}\left(n^{\dagger}(t)+\frac{1}{2}\right)+\frac{1}{2}\right]^{*}=m,
$$

where as before $[x]^{*}$ is the greatest positive integer less than $x$ or zero if such a positive integer does not exist. Now referring to subsection III.1 of Appendix III and putting $a_{r}=b_{r}(t), r=1, \ldots, p$, we then see from (4.13) that $\left(r^{\dagger}(t), n^{\dagger}(t)\right)$ renders soluble the equation $f_{r}(n)=m$; but Theorem III.1.2 states that this equation has a unique solution, namely $\left(r^{\dagger}, n^{\dagger}\right)$, and hence $r^{\dagger}(t)=r^{\dagger}, n^{\dagger}(t)=n^{\dagger}$.

We now arrive at our fundamental result, i.e. if we put $\bar{r}=r^{\dagger}\left(t_{1}\right)$ and $\bar{n}=n^{\dagger}\left(t_{1}\right)$, then $\nu_{\bar{r}}(\infty, t)=\bar{n}$ for $0<t \leqq t_{1}$. To see this, we fix an $r, 1 \leqq r \leqq p, r \neq \bar{r}$, and put $\phi_{r}(t)=\left\{\left(b_{\bar{r}}(t) / b_{r}(t)\right)^{1 / 2}\left(\bar{n}+\frac{1}{2}\right)-\frac{1}{2}\right\}$ for $t \geqq 0$; we remark that $\phi_{r}(t)$ is continuous and either strictly increasing or strictly decreasing for $t \geqq 0$. We also observe from (4.13) and the remarks immediately preceding (4.9) that $\phi_{r}\left(t_{1}\right)=\nu_{r}\left(\infty, t_{1}\right)$, and for some nonnegative integer $j$ not exceeding $m$ we have $\left[\phi_{r}\left(t_{1}\right)+1\right]^{*}=j$ and $j-1<\phi_{r}\left(t_{1}\right)<j$. We now assert that $\left[\phi_{r}(t)+1\right]^{*}=j$ for $0<t \leqq t_{1}$. For if this is not the case, then there is a $t_{2}, 0<t_{2}<t_{1}$, such that $\phi_{r}\left(t_{2}\right)=k$, where $k=j$ or $j-1$; but this implies that $\left(b_{\bar{r}}\left(t_{2}\right)\right)^{1 / 2}\left(\bar{n}+\frac{1}{2}\right)=\left(b_{r}\left(t_{2}\right)\right)^{1 / 2}\left(k+\frac{1}{2}\right)$ and therefore either $\left(b_{\bar{r}}\left(t_{2}\right) / b_{r}\left(t_{2}\right)\right)$ or $\left(b_{r}\left(t_{2}\right) / b_{\bar{r}}\left(t_{2}\right)\right)$ belongs to $R_{m}^{*}$, which is impossible. Since $r$ is arbitrary, we therefore conclude from (4.1.3) that

$$
\bar{n}+\sum_{r=1 ; r \neq \bar{r}}^{p}\left[\left(b_{\bar{r}}(t) / b_{r}(t)\right)^{1 / 2}\left(\bar{n}+\frac{1}{2}\right)+\frac{1}{2}\right]^{*}=m \text { for } 0<t \leqq t_{1} .
$$

Now referring to subsection III.1 of Appendix III, we see from (4.14) that if $0<t<t_{1}$ and $a_{r}=b_{r}(t), r=1, \ldots, p$, then $(\bar{r}, \bar{n})$ renders soluble the equation $f_{r}(n)=m$. But as we have stated above, this equation has the unique solution $\left(r^{\dagger}(t), n^{\dagger}(t)\right)$, and hence $r^{\dagger}(t)=\bar{r}, n^{\dagger}(t)=\bar{n}, 0<t \leqq t_{1}$.

Referring back to our original system (1.1-2), we now assert that $\nu_{\bar{r}}(\infty)=\bar{n}$, and hence from (4.5) we can determine $\nu_{r}(\infty), r=1, \ldots, p$. Our assertion follows immediately from the fact that $\lim _{\mu \rightarrow \infty}\left\{\Lambda_{m}(\mu, t)-\Lambda_{m}(\mu)\right\}=O(t)$ as $t \rightarrow 0[15$, Theorem 3.1] and hence $\left\{\left(b_{\bar{r}}(t)\right)^{1 / 2}\left(\bar{n}+\frac{1}{2}\right)-\left(b_{\bar{r}}\right)^{1 / 2}\left(\nu_{\bar{r}}(\infty)+\frac{1}{2}\right)\right\}=O(t)$ as $t \rightarrow 0$. This proves the theorem.

THEOREM 4.5. Let $\left(b_{i} / b_{j}\right) \in R$ for at least one pair of integers $(i, j)$, where $1 \leqq i$, $j \leqq p$ and $i \neq j$. From the set of integers $\{r\}_{r=1}^{p}$, let the subset $X$ be formed by demanding that $r \in X$ iff $\nu_{r}(\infty)$ is an integer. Then $X$ contains at least one element, and moreover, if $X$ contains precisely one element, and if we denote this element by $r^{*}$ and put $\nu_{r^{*}}(\infty)=n^{*}$, then (1.3) is valid with $A_{1, m}=G_{1,1}\left(r^{*}, n^{*}\right), A_{2, m}=0$, and $A_{3, m}=$ $G_{1,2}\left(r^{*}, n^{*}\right)$.

Proof. That $X$ contains at least one element follows from Theorem 4.2. Referring to the proof of Theorem 4.4 we see that if $X$ contains precisely one element, then 
it must be $\bar{r}$ and hence $\nu_{\bar{r}}(\infty)=\bar{n}$ is the only element of the set $\left\{\nu_{r}(\infty)\right\}_{r=1}^{p}$ which is an integer. Our theorem now follows by putting $r^{*}=\bar{r}, n^{*}=\bar{n}$ and arguing with (4.3) in precisely the same way as we did with (4.1).

From now on in this section we shall always assume that $\left(b_{i} / b_{j}\right) \in R$ for at least one pair of integers $(i, j)$, where $1 \leqq i, j \leqq p$ and $i \neq j$. Then in order to extend Theorem 4.5 to the case where $X$ contains more than one element, we can no longer restrict ourselves to the investigation of the asymptotic behaviour of just $\lambda_{m}(\mu)$. Hence for nonnegative integer $N \neq m$ and $\mu>0$ put $\Lambda_{N}(\mu)=\left(\lambda_{N}(\mu)+\mu A\right) / \mu^{1 / 2}$, and $\nu_{r}(\mu, N)=\left\{\left(\Lambda_{N}(\mu) / 2\left(b_{r}\right)^{1 / 2}\right)-\frac{1}{2}\right\}, r=1, \ldots, p$; and as in $\S 2$ we also note that there are constants $\mu_{N}$ and $\Lambda_{N}$, both greater than one, such that $0<\Lambda_{N}(\mu)<\Lambda_{N}$ for $\mu \geqq \mu_{N}$. Now it is clear that we may argue with $\lambda_{N}(\mu)$ in precisely the same way as we did with $\lambda_{m}(\mu)$, and by modifying (4.3) and Theorem 4.2 so as to apply to $\lambda_{N}(\mu)$, we then know that $\nu_{r}(\mu, N)$ tends to a finite limit as $\mu \rightarrow \infty, r=1, \ldots, p$, and at least one such limit is an integer. We shall in the sequel denote $\lim _{\mu \rightarrow \infty} \nu_{r}(\mu, N)$ by $\nu_{r}(\infty, N), r=1, \ldots, p$ (we remark that this notation will only be used if $N \neq m$; for $N=m$ we maintain our old notation, namely $\left.v_{r}(\infty), r=1, \ldots, p\right)$. We can then argue with (4.10) in precisely the same way as we did in the proof of Theorem 4.4 and determine the tuple $(\bar{r}(N), \bar{n}(N))$ satisfying $\nu_{\tilde{r}(N)}(\infty, N)=\bar{n}(N)$ and hence from the analogue of (4.5) determine the set $\left\{\nu_{r}(\infty, N)\right\}_{r=1}^{p}$.

Now returning to Theorem 4.5, denote the elements of the set $X$ by $\left\{r_{j}\right\}_{j=0}^{p^{*}}$, where $0 \leqq p^{*} \leqq p-1$, and put $\nu_{r_{j}}(\infty)=n_{j}, j=0, \ldots, p^{*}$. Here if $p^{*}=0$, then $r_{0}=\bar{r}$ and $n_{0}=\bar{n}$ (Theorem 4.4), while if $p^{*}>0$, then we shall always assume in the sequel, unless otherwise stated, that the $r_{j}, j=0, \ldots, p^{*}$, are arranged so that

$$
G_{1,2}\left(r_{0}, n_{0}\right) \leqq G_{1,2}\left(r_{1}, n_{1}\right) \leqq \cdots \leqq G_{1,2}\left(r_{p^{*}}, n_{p^{*}}\right),
$$

where if $G_{1,2}\left(r_{i-1}, n_{i-1}\right)=G_{1,2}\left(r_{i}, n_{i}\right)$ for $1 \leqq i \leqq p^{*}$, then $h_{r_{i-1}}<h_{r_{i}}$.

THEOREM 4.6. Under the hypothesis of Theorem 4.5 assume that $X$ contains at least two elements. Let the elements of $X$ be denoted by $\left\{r_{j}\right\}_{j=0}^{p^{*}}$, where $1 \leqq p^{*} \leqq p-1$, and put $\nu_{r_{j}}(\infty)=n_{j}, j=0, \ldots, p^{*}$; here the $r_{j}, j=0, \ldots, p^{*}$, are arranged so that $(4.15)$ is satisfied. Let $s=m-N_{1}$, where if $m=0$, or if $m>0$ and $\nu_{1}(\infty, m-1)<\nu_{1}(\infty)$, then $N_{1}=m$, while if $m>0$ and $\nu_{1}(\infty, m-1)=\nu_{1}(\infty)$, then $N_{1}$ is the smallest integer satisfying $\nu_{1}\left(\infty, N_{1}\right)=v_{1}(\infty)$. Then $0 \leqq s \leqq p^{*}$, and (1.3) is valid with $A_{1, m}=G_{1,1}\left(r_{s}, n_{s}\right)$, $A_{2, m}=0$, and $A_{3, m}=G_{1,2}\left(r_{s}, n_{s}\right)$.

We shall not prove Theorem 4.6 directly; indeed this theorem follows immediately from Lemma 4.2 and Theorem 4.7.

In order to establish the validity of Theorem 4.6 we shall have to analyse (4.14) in greater detail. To this end we return to the proof of Theorem 4.4 and write (4.14) in the form

$$
\bar{n}+S_{1}+S_{2}=m \text { for } 0<t \leqq t_{1},
$$


where

$$
\begin{aligned}
& S_{1}=\sum_{j=0 ; r_{j} \neq \bar{r}}^{p^{*}}\left[\phi_{r_{j}}(t)+1\right]^{*} \quad \text { if } p^{*}>0, \quad S_{1}=0 \quad \text { otherwise, } \\
& S_{2}=\sum_{r=1 ; r \notin X}^{p}\left[\phi_{r}(t)+1\right]^{*} \quad \text { if } p^{*}<p-1, \quad S_{2}=0 \quad \text { otherwise, }
\end{aligned}
$$

and all other terms are defined in the proof of Theorem 4.4. Here we have suppressed the $t$, and have written $S_{1}$ for $S_{1}(t)$ and $S_{2}$ for $S_{2}(t)$, since we know from the proof of Theorem 4.4 that both $S_{1}(t)$ and $S_{2}(t)$ are constant in $0<t \leqq t_{1}$. Moreover, we also know that $S_{2}(t)$ is constant in $\left[0, t_{1}\right]$; for if $p^{*}<p-1$ and $r \notin X$, then $\phi_{r}(0)=\nu_{r}(\infty)$ is not an integer, and so from the proof of Theorem 4.4 we see that $\phi_{r}(t)$ cannot assume integral values in the interval $\left[0, t_{1}\right]$. Similarly if $p^{*}>0$, $0 \leqq j \leqq p^{*}$, and $r_{j} \neq \bar{r}$, then we know that $\phi_{r_{j}}(0)=n_{j}$ and $\phi_{r_{j}}(t)$ does not assume integral values in $0<t \leqq t_{1}$ and is either strictly increasing or strictly decreasing in $\left[0, t_{1}\right]$; hence $\left[\phi_{r_{j}}(t)+1\right]^{*}=n_{j}$ or $n_{j}+1$ for $0<t \leqq t_{1}$, and since $j$ is arbitrary we see that $\sum_{j=0}^{p^{*}} n_{j} \leqq \bar{n}+S_{1} \leqq p^{*}+\sum_{j=0}^{p^{*}} n_{j}$.

Let $P(m)$ denote the set of integers $\left\{N_{1}+j\right\}_{j=0}^{p^{*}}$, where $N_{1}=\sum_{j=0}^{p^{*}} n_{j}+S_{2}$; we observe from (4.16) and the above remarks that $m \in P(m)$.

Lemma 4.1. For each $N \in P(m)$ there is precisely one $k, 0 \leqq k \leqq p^{*}$, such that

$$
N=n_{k}+\sum_{r=1 ; r \neq r_{k}}^{p}\left[\left(b_{r_{k}}(t) / b_{r}(t)\right)^{1 / 2}\left(n_{k}+\frac{1}{2}\right)+\frac{1}{2}\right]^{*}
$$

for all positive $t$ sufficiently small.

Proof. If $p^{*}=0$, then $m$ is the only member of $P(m)$ and $r_{0}=\bar{r}$ is the only member of $X$ and our lemma follows directly from (4.16) (with $\bar{n}=n_{0}$ and $S_{1}=0$ ).

We now consider the case $p^{*}>0$. Then in order to avoid a proliferation of notation, assume for the remainder of this proof that the elements of the set $X$ have been relabelled (if necessary) so that the $b_{r j}, j=0, \ldots, p^{*}$, now satisfy the relationship $b_{r_{0}} \leqq b_{r_{1}} \leqq \cdots \leqq b_{r_{p *}}$, where if, for $1 \leqq i \leqq p^{*}, b_{r_{i-1}}=b_{r_{i}}$, then $h_{r_{i-1}}<h_{r_{i}}$. Also from the definition of the functions $\left\{b_{r}(t)\right\}_{r=1}^{p}$ given in the proof of Theorem 4.4 , it is a simple matter to verify that if $1 \leqq i, j \leqq p$ and $i \neq j$, then in the interval $t \geqq 0,\left(b_{i}(t) / b_{j}(t)\right)$ is (1) strictly increasing if $b_{j}>b_{i},(2)$ strictly increasing if $b_{j}=b_{i}$ and $h_{j}>h_{i}$, (3) strictly decreasing if $b_{j}=b_{i}$ and $h_{j}<h_{i}$, and (4) strictly decreasing if $b_{j}<b_{i}$.

Now for $0 \leqq k \leqq p^{*}, 1 \leqq r \leqq p$, and $r \neq r_{k}$, put $\phi_{k, r}(t)=\left\{\left(b_{r_{k}}(t) / b_{r}(t)\right)^{1 / 2}\left(n_{k}+\frac{1}{2}\right)-\frac{1}{2}\right\}$ for $t \geqq 0$. We observe that $\phi_{k, r}(t)$ is continuous and either strictly increasing or strictly decreasing in $t \geqq 0$. If $p^{*}<p-1$ and $r \notin X$, then we also know that $\phi_{k, r}(0)$ $=\phi_{r}(0)=\nu_{r}(\infty)$, which is not an integer (see (4.16)), and so for all $t$ sufficiently small

$$
\sum_{r=1 ; r \notin X}^{p}\left[\phi_{k, r}(t)+1\right]^{*}=\sum_{r=1 ; r \notin X}^{p}\left[\phi_{r}(t)+1\right]^{*}=S_{2} .
$$


If $k<p^{*}$ and $k<j \leqq p^{*}$, then $\phi_{k, r_{j}}(t)$ is strictly increasing for $t \geqq 0$ (see the remarks above) and $\phi_{k, r_{j}}(0)=n_{j}$. If $k>0$ and $0 \leqq j<k$, then $\phi_{k, r_{j}}(t)$ is strictly decreasing for $t \geqq 0$ and $\phi_{k, r_{j}}(0)=n_{j}$. We therefore conclude that

$$
n_{k}+\sum_{r=1 ; r \neq r_{k}}^{p}\left[\phi_{k, r}(t)+1\right]^{*}=k+\sum_{j=0}^{p^{*}} n_{j}+S_{2}=k+N_{1}
$$

for all positive $t$ sufficiently small. This proves the lemma.

Referring to the remarks immediately following Theorem 4.5 , it is then clear that for nonnegative integer $N \neq m$ we may argue with $\lambda_{N}(\mu)$ as above and define the set of integers $P(N)$ in an analogous fashion to $P(m)$. We then have

Lemma 4.2. (1) If $N \in P(m)$ and $N \neq m$, then $v_{r}(\infty, N)=v_{r}(\infty)$ for $r=1, \ldots, p$ and the sets $P(N)$ and $P(m)$ are identical.

(2) $P(m)$ is precisely the set of integers which contains $m$ and all integers $N \neq m$ for which $\nu_{r}(\infty, N)=\nu_{r}(\infty)$ for $r=1, \ldots, p$. Hence the integers $N \neq m$ for which $\nu_{r}(\infty, N)=\nu_{r}(\infty)$ for $r=1, \ldots, p$ form together with $m$ a set of $\left(p^{*}+1\right)$ consecutive integers.

(3) If $m>0$ and $0 \leqq N<m$, then $\nu_{r}(\infty, N) \leqq \nu_{r}(\infty, N+1) \leqq \cdots \leqq \nu_{r}(\infty, m-1)$ $\leqq \nu_{r}(\infty)$ for $r=1, \ldots, p$; and if for at least one $r, 1 \leqq r \leqq p, \nu_{r}(\infty, N)=\nu_{r}(\infty)$, then $N,(N+1), \ldots,(m-1)$ all belong to $P(m)$.

Proof. If $N \in P(m)$ and $N \neq m$, then the arguments used in the proof of Theorem 4.4 show immediately that the analogue of (4.14) for $\lambda_{N}(\mu)$ is precisely (4.17). So we conclude from the proof of Theorem 4.4 that $\bar{r}(N)=r_{k}, \bar{n}(N)=n_{k}, \nu_{r_{k}}(\infty, N)$ $=v_{r_{k}}(\infty)$, and hence from (4.5) and its analogue for $\lambda_{N}(\mu)$, we see that $\nu_{r}(\infty, N)=$ $\nu_{r}(\infty), r=1, \ldots, p$.

We now show that if $N$ is a nonnegative integer, $N \neq m$, and if for at least one $r$, $1 \leqq r \leqq p, \nu_{r}(\infty, N)=\nu_{r}(\infty)$, then $N \in P(m)$. First, we observe from (4.5) and its analogue for $\lambda_{N}(\mu)$, that our hypothesis implies $\nu_{r}(\infty, N)=\nu_{r}(\infty), r=1, \ldots, p$. Then the arguments used in the proof of Theorem 4.4 show that for precisely one $k$, $0 \leqq k \leqq p^{*}, \bar{r}(N)=r_{k}, \bar{n}(N)=n_{k}$, and (4.17) is valid for this $k$ for all positive $t$ sufficiently small. Hence from Lemma 4.1 we conclude that $N \in P(m)$.

These results, together with our definition of $P(m)$, prove part (2) of our lemma. Since part (2) can be modified to apply to $P(N)$, part (1) of our lemma also follows.

To prove part (3), assume that there is a $j, 1 \leqq j \leqq p$, for which $v_{j}(\infty, N)>v_{j}(\infty)$. Then (4.5) and its analogue for $\lambda_{N}(\mu)$ imply that $\nu_{r}(\infty, N)>\nu_{r}(\infty)$ for $r=1, \ldots, p$. Now from (4.14) we see that $\sum_{r=1}^{p}\left[\nu_{r}(\infty, t)+1\right]^{*}=m$ for $0<t \leqq t_{1}$, where $\nu_{r}(\infty, t)$ is continuous in $\left[0, t_{1}\right]$ and $\nu_{r}(\infty, 0)=v_{r}(\infty), r=1, \ldots, p$. The analogue of this for $\lambda_{N}(\mu)$ (obtained by replacing $\bar{n}$ by $\bar{n}(N)$ and $\bar{r}$ by $\bar{r}(N)$ in (4.14) for all $t$ sufficiently small) is $\sum_{r=1}^{p}\left[\nu_{r}(\infty, N, t)+1\right]^{*}=N$ for $0<t \leqq t_{2}$ for some $t_{2}>0$, where $\nu_{r}(\infty, N, t)$ is continuous in $\left[0, t_{2}\right]$ and $\nu_{r}(\infty, N, 0)=\nu_{r}(\infty, N), r=1, \ldots, p$. If $1 \leqq r \leqq p$, then $\left(\nu_{r}(\infty, N, t)+1\right)>\left(\nu_{r}(\infty, t)+1\right)$ and $\left[\nu_{r}(\infty, N, t)+1\right]^{*} \geqq\left[\nu_{r}(\infty, t)+1\right]^{*}$ for all $t$ sufficiently small. Thus $\sum_{r=1}^{p}\left[\nu_{r}(\infty, N, t)+1\right]^{*} \geqq m>N$ for all $t$ sufficiently small, which 
is impossible; hence $\nu_{r}(\infty, N) \leqq \nu_{r}(\infty), r=1, \ldots, p$. We can also argue in the same way to show that $\nu_{r}(\infty, N) \leqq \nu_{r}(\infty, N+1) \leqq \cdots \leqq \nu_{r}(\infty, m-1) \leqq \nu_{r}(\infty), r=1, \ldots, p$, and so part (3) of our lemma follows from the above results.

The connection between the set $P(m)=\left\{N_{1}+j\right\}_{j=0}^{p^{*}}$ and the eigenvalues of (1.1-2) is demonstrated in the following theorem. Since the case $p^{*}=0$ has already been considered in Theorem 4.5, we shall only consider the case $p^{*}>0$. We might also point out that in this theorem particular attention must be paid to the ordering described in (4.15).

THEOREM 4.7. If $p^{*}>0$, then

$$
\lambda_{N_{1}+j}(\mu)=-\mu A+2\left(b_{r_{j}}\right)^{1 / 2}\left(n_{j}+\frac{1}{2}\right) \mu^{1 / 2}+G_{1,2}\left(r_{j}, n_{j}\right)+o(1)
$$

as $\mu \rightarrow \infty, j=0, \ldots, p^{*}$.

Proof. For convenience of notation we shall in this proof write $w_{j}$ for $G_{1,2}\left(r_{j}, n_{j}\right)$, $j=0, \ldots, p^{*}$, and denote by $B$ the subset of the reals whose elements are precisely the numbers $w_{j}, j=0, \ldots, p^{*}$. Then returning to our investigation of the asymptotic behaviour of $\lambda_{m}(\mu)$, we continue with the arguments which terminated with (4.15). Referring to (4.3), we see that there is a $\mu^{\dagger}>0$ such that for each $\mu \geqq \mu^{\dagger}$,

$$
\left|K_{r_{k}}(\mu) / \cos \pi\left(v_{r_{k}}+1\right)\right|<\exp \left\{-\Delta \mu^{1 / 2} /\left(p^{*}+1\right)\right\}
$$

for at least one $k, 0 \leqq k \leqq p^{*}$. Hence from (4.4), Appendix I, and by arguing with our inverse function theorem as in the proof of Theorem 4.1, we see that for any $\varepsilon>0$ there is a $\mu(\varepsilon)>0$ such that for each $\mu \geqq \mu(\varepsilon),\left|\mu^{1 / 2} g(\mu)-w_{k}\right|<\varepsilon$ for at least one $k$, where $g(\mu)=2\left(b_{r_{0}}\right)^{1 / 2}\left(\nu_{r_{0}}(\mu)-n_{0}\right)=2\left(b_{r_{j}}\right)^{1 / 2}\left(\nu_{r_{j}}(\mu)-n_{j}\right), j=0, \ldots, p^{*}$. Since $\varepsilon$ is arbitrary and $g(\mu)$ is continuous for $\mu>0$, we therefore conclude that $\mu^{1 / 2} g(\mu)$ tends to precisely one element of $B$ as $\mu \rightarrow \infty$. But from $\S 2$ this implies that $\left\{\lambda_{m}(\mu)+\mu A-\mu^{1 / 2} g_{0}\right\}$ tends to precisely one element of $B$ as $\mu \rightarrow \infty$, where

$$
g_{0}=2\left(b_{r_{0}}\right)^{1 / 2}\left(n_{0}+\frac{1}{2}\right)=2\left(b_{r_{j}}\right)^{1 / 2}\left(n_{j}+\frac{1}{2}\right), \quad j=0, \ldots, p^{*} \text {. }
$$

On the other hand, we see from part (1) of Lemma 4.2 that if $N \in P(m)$ and $N \neq m$, then the above arguments applied to the analogue of (4.3) for $\lambda_{N}(\mu)$ lead to the conclusion that $\left\{\lambda_{N}(\mu)+\mu A-\mu^{1 / 2} g_{0}\right\}$ tends to precisely one element of $B$ as $\mu \rightarrow \infty$.

Hence for each $j, 0 \leqq j \leqq p^{*}$, we conclude that $M_{j}(\mu)=\left\{\lambda_{N_{1}+j}(\mu)+\mu A-\mu^{1 / 2} g_{0}\right\}$ tends to precisely one element of $B$ as $\mu \rightarrow \infty$. If $B$ contains precisely one element, that is to say, all the $w_{j}, j=0, \ldots, p^{*}$, are equal, then our theorem is proved for this case. So from now on we shall assume that $B$ contains at least two elements. Since $M_{0}(\mu)<M_{1}(\mu)<\cdots<M_{p^{*}}(\mu)$ for $\mu>0$, we see that if we put $\lim _{\mu \rightarrow \infty} M_{j}(\mu)$ $=M_{j}(\infty), j=0, \ldots, p^{*}$, then

$$
M_{0}(\infty) \leqq M_{1}(\infty) \leqq \cdots \leqq M_{p^{*}}(\infty)
$$

It only remains to show that $M_{j}(\infty)=w_{j}, j=0, \ldots, p^{*}$. 
Assume now that the $w_{j}, j=0, \ldots, p^{*}$, are all distinct; then for each $N \in P(m)$ there is precisely one integer $k(N)$, where $0 \leqq k(N) \leqq p^{*}$, such that

$$
M_{N-N_{1}}(\infty)=w_{k(N)} \text {. }
$$

Putting $r^{*}=r_{k(m)}$ and $n^{*}=n_{k(m)}$, we will proceed to the eigenfunctions corresponding to $\lambda_{m}(\mu)$.

If in applying the results of $\S 3$ to our present section we replace $y_{1}(x, \mu)$ by $y_{1, r}(x, \mu)$ and $y_{2}(x, \mu)$ by $y_{2, r}(x, \mu),\left|x-h_{r}\right| \leqq \mu^{-3 / 16} /\left(4 b_{r}\right)^{1 / 4}, r=1, \ldots, p$ (see Theorem 4.2) and if we denote by $Y(x, \mu)$ an eigenfunction of (1.1-2) corresponding to $\lambda_{m}(\mu)$, then an investigation of the determinant which gives rise to (4.3), together with the results stated in [18, Theorem 24.1, p. 124] and [13, pp. 347-348], shows that for all sufficiently large values of $\mu$,

$$
\begin{aligned}
Y(x, \mu) & =K_{1, r}(\mu) y_{1, r}(x, \mu)+K_{2, r}(\mu) y_{2, r}(x, \mu) \\
& \text { in }\left|x-h_{r}\right| \leqq \mu^{-3 / 16} /\left(4 b_{r}\right)^{1 / 4}, \text { for } r=1, \ldots, p \text { and } r \neq r^{*}, \\
= & K(\mu)\left[\left((2 \pi)^{1 / 2} / n^{*} !\right) D_{n^{*}}(\xi)\left(1+v_{1, m}(\xi, \mu)\right)+\mu^{-1 / 4} v_{2, m}(\xi, \mu) U_{1}\left(\xi, n^{*}\right)\right] \\
& \text { in }\left|x-h_{r^{*}}\right| \leqq \mu^{-15 / 64} /\left(4 b_{r^{*}}\right)^{1 / 4},
\end{aligned}
$$

where $\xi=\left(4 \mu b_{r^{*}}\right)^{1 / 4}\left(x-h_{r^{*}}\right), D_{n^{*}}(\xi), U_{1}\left(\xi, n^{*}\right)$ are defined in Appendix I, and as $\mu \rightarrow \infty, v_{1, m}\left(t \mu^{1 / 64}, \mu\right)=O\left(\mu^{-13 / 64}\right), v_{2, m}\left(t \mu^{1 / 64}, \mu\right)=O(1)$, uniformly in $|t| \leqq 1$, and $\left(K_{i, r}(\mu) / K(\mu)\right)=O\left(\exp \left\{-\Delta^{\dagger} \mu^{1 / 2}\right\}\right), i=1,2, r=1, \ldots, p, r \neq r^{*}$, where $\Delta^{\dagger}$ is a positive constant.

Put $\Lambda=\max _{N \in P(m)}\left\{\Lambda_{N}\right\}$ (see $\S 2$ and the remarks immediately following Theorem 4.5), $b^{*}=\max _{1 \leqq r \leqq p}\left\{b_{r}\right\}, \delta^{\dagger}=((\Lambda+Q) / b \theta)^{1 / 2}, \delta^{*}=\left(4 b^{*}\right)^{1 / 4} \delta^{\dagger}$, and choose the positive constants $\delta$ and $\mu^{\dagger}$ so that $\delta>\delta^{*}$ and $\mu^{\dagger}>\max \left\{2,\left((4 b)^{1 / 4} h\right)^{-64 / 15}, \delta^{64}\right\}$ (and where all terms that are not defined above are defined in the proof of Theorem 4.3). If $\phi_{m}(x, \mu)$ is the eigenfunction of (1.1-2) corresponding to $\lambda_{m}(\mu)$ such that $\int_{0}^{1} \phi_{m}^{2}(x, \mu) d x$ $=1$, then (4.21), the results of subsection 3.1, and [15, Theorem 2.1] show that the absolute maximum of $\left|\phi_{m}(x, \mu)\right|$ in $[0,1]$ is attained in the interval $\left|x-h_{r^{*}}\right| \leqq \mu^{-1 / 4} \delta^{\dagger}$ for all sufficiently large values of $\mu$. Hence from (4.21) and [15, Theorem 2.3 and the remarks immediately preceding Theorem 2.5$]$, we see that there exists a sequence of values of $\mu,\left\{\mu_{l}\right\}_{l=1}^{\infty}, \mu^{\dagger}<\mu_{1}<\mu_{2}<\cdots, \lim _{l \rightarrow \infty} \mu_{l}=\infty$, such that for all sufficiently large values of $l$ and with $\phi_{m}\left(x, \mu_{l}\right)=Z_{m}\left(\xi, \mu_{l}\right), \xi=\left(4 \mu_{l} b_{r^{*}}\right)^{1 / 4}\left(x-h_{r^{*}}\right)$,

$$
Z_{m}\left(\xi, \mu_{l}\right)=k_{m}\left(\mu_{l}\right)\left[D_{n^{*}}(\xi)\left(1+v_{1, m}\left(\xi, \mu_{l}\right)\right)+\left(n^{*} ! /(2 \pi)^{1 / 2}\right) \mu_{l}^{-1 / 4} v_{2, m}\left(\xi, \mu_{l}\right) U_{1}\left(\xi, n^{*}\right)\right],
$$

$$
\begin{aligned}
& =k_{m}\left(\mu_{l}\right) D_{n^{*}}(\xi)\left[1+v_{3, m}\left(\xi, \mu_{l}\right)\right], \quad \delta \leqq \xi \leqq \mu_{l}^{1 / 64}, \\
& =k_{m}\left(\mu_{l}\right) D_{n^{*}}(\xi)\left[1+v_{4, m}\left(\xi, \mu_{l}\right)\right], \quad-\mu_{l}^{1 / 64} \leqq \xi \leqq-\delta \text {, }
\end{aligned}
$$

and

$$
\left(\left(Z_{m}\left(t \mu_{l}^{1 / 64}, \mu_{l}\right)\right)^{2}+\mu_{l}^{1 / 2}\left(Z_{m}^{\prime}\left(t \mu_{l}^{1 / 64}, \mu_{l}\right)\right)^{2}\right)^{1 / 2}=O\left(\exp \left\{-\Delta_{m}^{*} \mu_{l}^{1 / 32}\right\}\right)
$$

as $l \rightarrow \infty$, uniformly in $|t| \geqq 1$ (and of course $t$ is restricted so that $0 \leqq x \leqq 1$ ), where $'=d / d \xi, \Delta_{m}^{*}$ is a positive constant, $v_{1, m}\left(\xi, \mu_{l}\right), v_{2, m}\left(\xi, \mu_{l}\right)$ as above, $v_{i, m}\left(t \mu_{l}^{1 / 64}, \mu_{l}\right)$ $=O\left(\mu_{l}^{-13 / 64}\right)$ as $l \rightarrow \infty$, uniformly in $\delta \mu_{l}^{-1 / 64} \leqq|t| \leqq 1, i=3,4$, and

$$
\mu_{l}^{-1 / 8} k_{m}\left(\mu_{l}\right)=\left(\left(4 b_{r^{*}}\right)^{1 / 4} /(2 \pi)^{1 / 2}\left(n^{*} !\right)\right)^{1 / 2}\left[1+O\left(\mu_{l}^{-13 / 64}\right)\right] \quad \text { as } l \rightarrow \infty .
$$


If for each $N \in P(m)$ and $N \neq m$ we denote by $\phi_{N}(x, \mu)$ the eigenfunction of (1.1-2) corresponding to $\lambda_{N}(\mu)$ and satisfying $\int_{0}^{1} \phi_{N}^{2}(x, \mu) d x=1$, then by a selection of a subsequence of the $\mu_{l}, l=1,2, \ldots$, and relabelling suitably (see the remarks immediately preceding $(4.22 . \mathrm{m})$ ) we can also assume that $(4.22 . N)$ (obtained from (4.22.m) by replacing $r^{*}$ by $r_{k(N)}, n^{*}$ by $n_{k(N)}$, and $m$ by $\left.N\right)$ is valid for $\phi_{N}(x, \mu)$.

Now we know from (4.19) and (4.20) that $w_{k\left(N_{1}\right)} \leqq w_{k\left(N_{1}+1\right)} \leqq \cdots \leqq w_{k\left(N_{1}+p^{*}\right)}$. On the other hand, from the orthogonal properties of the eigenfunctions, we see from equations $\left(4.22 . N_{1}\right), \ldots,\left(4.22 . N_{1}+p^{*}\right),[13$, pp. 350-351], and [15, proof of Theorem 2.5] that the $k(N), N=N_{1}, \ldots,\left(N_{1}+p^{*}\right)$ must all be distinct. Since $w_{0}<w_{1}$ $<\cdots<w_{p^{*}}$, we therefore conclude that $k\left(N_{1}+j\right)=j, j=0, \ldots, p^{*}$. Hence $M_{j}(\infty)$ $=w_{k\left(N_{1}+j\right)}=w_{j}, j=0, \ldots, p^{*}$, and our theorem is proved for this case.

To prove our theorem for the case where the $w_{j}, j=0, \ldots, p^{*}$, are not all distinct, we replace $(1.1)$ by the perturbed equation

$$
y^{\prime \prime}(x)+(\lambda+\mu a(x)+q(x, t)) y(x)=0, \quad 0 \leqq x \leqq 1, \quad '=d / d x, \quad t>0,
$$

where $q(x, t)=q(x)-t x$. If for $N \in P(m)$ we denote the $N$ th eigenvalue of system (4.23), (1.2) by $\lambda_{N}(\mu, t)$, and denote by $B(t)$ the subset of the reals whose elements are precisely the $w_{j}(t)=w_{j}+t h_{r j}, j=0, \ldots, p^{*}$ (see (4.4) and Appendix I), then our above calculations tell us that $M_{N-N_{1}}(\mu, t)=\left\{\lambda_{N}(\mu, t)+\mu A-\mu^{1 / 2} g_{0}\right\}$ tends to precisely one element of $B(t)$ as $\mu \rightarrow \infty$. On the other hand, for all $t$ sufficiently small we see from (4.15) that $w_{0}(t)<w_{1}(t)<\cdots<w_{p^{*}}(t)$; and so from above we conclude that $\lim _{\mu \rightarrow \infty} M_{j}(\mu, t)=w_{j}(t), j=0, \ldots, p^{*}$, for all $t$ sufficiently small.

Consider now the differential equation in the two parameters $\gamma$ and $\mu$,

$$
\begin{aligned}
y^{\prime \prime}(x)+\left(\gamma+g_{0} \mu^{1 / 2}-\mu(A-a(x))+\right. & q(x)) y(x)=0, \\
& 0 \leqq x \leqq 1, \quad,=d / d x, \quad \mu>0,
\end{aligned}
$$

and where all terms are as previously defined. For each positive $\mu$ denote the eigenvalues of (4.24), (1.2) by $\left\{\gamma_{n}(\mu)\right\}_{n=0}^{\infty}$, where $\gamma_{0}(\mu)<\gamma_{1}(\mu)<\cdots, \lim _{n \rightarrow \infty} \gamma_{n}(\mu)$ $=\infty$, and where an eigenfunction corresponding to $\gamma_{n}(\mu)$ has precisely $n$ zeros in $(0,1)$. Now it is clear that for $j=0, \ldots, p^{*}, \gamma_{N_{1}+j}(\mu)=M_{j}(\mu)$; and hence

$$
\lim _{\mu \rightarrow \infty} \gamma_{N_{1}+j}(\mu)=M_{j}(\infty)
$$

If $N^{*}=\left(N_{1}+p^{*}+1\right)$, then we may also argue with $\lambda_{N^{*}}(\mu)$ in the same way as we did with $\lambda_{m}(\mu)$ to show that $\left\{\lambda_{N^{*}}(\mu)+\mu A-2\left(b_{1} \mu\right)^{1 / 2}\left(\nu_{1}\left(\infty, N^{*}\right)+\frac{1}{2}\right)\right\}$ tends to a finite limit as $\mu \rightarrow \infty$. Then by arguing as in the proof of part (3) of Lemma 4.2, it is a simple matter to verify that $\nu_{1}\left(\infty, N^{*}\right) \geqq \nu_{1}(\infty)$; and since $N^{*} \notin P(m)$, we conclude from this lemma that $\nu_{1}\left(\infty, N^{*}\right)>\nu_{1}(\infty)$. Since

$$
\gamma_{N^{*}}(\mu)=\left[\lambda_{N^{*}}(\mu)+\mu A-2\left(b_{1} \mu\right)^{1 / 2}\left(\nu_{1}\left(\infty, N^{*}\right)+\frac{1}{2}\right)+2\left(b_{1} \mu\right)^{1 / 2}\left(\nu_{1}\left(\infty, N^{*}\right)-\nu_{1}(\infty)\right)\right],
$$

we therefore conclude that $\lim _{\mu \rightarrow \infty} \gamma_{N^{*}}(\mu)=\infty$. Similarly if $N_{1}>0$, then we can also show that $\lim _{\mu \rightarrow \infty} \gamma_{N_{1}-1}(\mu)=-\infty$. 
With $q(x, t)$ as defined in (4.23), replace (4.24) by the perturbed equation

$$
\begin{aligned}
& y^{\prime \prime}(x)+\left(\gamma+g_{0} \mu^{1 / 2}-\mu(A-a(x))+q(x, t)\right) y(x)=0, \\
& 0 \leqq x \leqq 1, \quad=d / d r, \quad \mu>0, \quad t>0,
\end{aligned}
$$

and for each positive $\mu$ denote the eigenvalues of system (4.25), (1.2) by $\gamma_{n}(\mu, t)$, $n=0,1, \ldots$ Then it is clear that for $j=0, \ldots, p^{*}, \gamma_{N_{1}+j}(\mu, t)=M_{j}(\mu, t)$, and hence $\lim _{\mu \rightarrow \infty} \gamma_{N_{1}+j}(\mu, t)=w_{j}(t)$ for all $t$ sufficiently small.

Now we may argue with systems (4.24), (1.2) and (4.25), (1.2) as in [19, pp. 231233], [15, Theorem 3.1] to show that $\lim _{\mu \rightarrow \infty}\left\{\gamma_{N_{1}+j}(\mu, t)-\gamma_{N_{1}+j}(\mu)\right\}=O(t)$ as $t \rightarrow 0$, $j=0, \ldots, p^{*}$. Thus $\left\{w_{j}+t h_{r_{j}}-M_{j}(\infty)\right\}=O(t)$ as $t \rightarrow 0, j=0, \ldots, p^{*}$, and hence our theorem follows.

5. Main results for the case $a(0)=A$ and $a(1)<A$.

5.0. Introduction. In this section we consider the case where $a(x)=A$ at precisely the finite set of points $\left\{h_{r}\right\}_{r=0}^{p}$, where $p \geqq 0$ and $0=h_{0}<h_{1}<\cdots<h_{p}<1$. Then throughout this section, unless otherwise stated, we shall consider the integer $m$ fixed as before and use the results of $\S 3$ to verify (1.3). Since for the most part the arguments of $\$ 4$ can be modified so as to apply to the work of this section, we shall therefore content ourselves here with mainly stating our results and only elaborating on points where the arguments of $\S 4$ are no longer valid. Finally, the two cases (1) $\alpha=0$, (2) $\alpha \neq 0$ will be treated separately; and for terminology we refer to $\S \S 2$ and 4.

5.1. The case $\alpha=0$. An argument analogous to that used in Theorem 4.2, together with the results of $\S 3$, show that if $p>0$ (and with obvious modifications if $p=0$ ) then

$$
K_{0}^{*}(\mu) \prod_{r=1}^{p} K_{r}(\mu)=o\left(\exp \left\{-\Delta \mu^{1 / 2}\right\}\right) \quad \text { as } \mu \rightarrow \infty,
$$

where

$$
K_{0}^{*}(\mu)=\sin \pi\left(\nu_{0}+1\right) / 2-\cos \pi\left(\nu_{0}+1\right) / 2\left[c_{0} A_{2}\left(\nu_{0}\right) \mu^{-1 / 4} / 3+\left(W_{0}\left(\nu_{0}\right)+f_{0}^{*}(\mu)\right) \mu^{-1 / 2} / 2\right],
$$

$f_{0}^{*}(\mu)=o(1)$ as $\mu \rightarrow \infty, W_{0}(x)$ is given by (4.4) with $r=0$, and all other terms are defined in the statements immediately following (4.3).

THEOREM 5.1.1. If $p=0$, then (1.3) is valid with $A_{1, m}=G_{2,1}(0, m), A_{2, m}=G_{2,2}(0, m)$, and $A_{3, m}=G_{2,3}(0, m)$.

From now on in this section we shall assume that $p>0$. Then by arguing with (5.1.1) as in Theorem (4.2) we have:

Theorem 5.1.2. As $\mu \rightarrow \infty, \nu_{r}$ tends to a finite limit, $r=0, \ldots, p$, and at least one such limit is an integer.

In the sequel we shall denote $\lim _{\mu \rightarrow \infty} \nu_{r}$ by $\nu_{r}(\infty), r=0, \ldots, p$; and we observe from $\S 2$ that if $\nu_{r}(\infty)$ is an integer, then it must be a nonnegative integer. Moreover, 
we see from (5.1.1) that if $\nu_{0}(\infty)$ is not an odd integer, then at least one element of the set $\left\{\nu_{r}(\infty)\right\}_{r=1}^{p}$ is an integer. Also from $\S 2$ and Theorem 5.1.2 we see that

$$
\left(b_{0}\right)^{1 / 2}\left(\nu_{0}(\infty)+\frac{1}{2}\right)=\left(b_{r}\right)^{1 / 2}\left(\nu_{r}(\infty)+\frac{1}{2}\right), \quad r=0, \ldots, p .
$$

THEOREM 5.1.3. If $\left(b_{i} / b_{j}\right) \notin R$ for $i, j=0, \ldots, p$ and $i \neq j$, then the elements of the set $\left\{\nu_{r}(\infty)\right\}_{r=0}^{p}$ can be determined. Furthermore, precisely one element of this set is an integer, and $\nu_{0}(\infty)$ is either an odd integer or nonintegral. If we denote by $\nu_{r^{*}}(\infty)$ the element which is an integer and

(1) put $\nu_{0}(\infty)=2 n^{*}+1$ if $r^{*}=0$, then (1.3) is valid with $A_{1, m}=G_{2,1}\left(0, n^{*}\right), A_{2, m}$ $=G_{2,2}\left(0, n^{*}\right)$, and $A_{3, m}=G_{2,3}\left(0, n^{*}\right)$;

(2) put $\nu_{r^{*}}(\infty)=n^{*}$ if $r^{*} \neq 0$, then (1.3) is valid with $A_{1, m}=G_{1,1}\left(r^{*}, n^{*}\right), A_{2, m}=0$, and $A_{3, m}=G_{1,2}\left(r^{*}, n^{*}\right)$.

Proof. First we observe from (5.1.2) that only one element of the set $\left\{\nu_{r}(\infty)\right\}_{r=0}^{p}$ can be an integer; and from our above remarks we also see that if $\nu_{0}(\infty)$ is integral then it must be odd. We now assert that in order to determine the tuple $\left(r^{*}, n^{*}\right)$ stated in our theorem, we need only put $a_{r}=b_{r}, r=0, \ldots, p$, in subsection III.2 of Appendix III, and with $f_{r}(n)$ given by equation (III.2.1), solve for $\left(r^{\dagger}, n^{\dagger}\right)$ the equation $f_{r}(n)=m$ (see Theorem III.2.1) and then we must have $r^{*}=r^{\dagger}, n^{*}=n^{\dagger}$. This assertion is proved by arguing with (5.1.1) and (5.1.2) as in the proof of Theorem 4.3. Our theorem then follows from (5.1.1) and (5.1.2).

THEOREM 5.1.4. If $\left(b_{i} / b_{j}\right) \in R$ for at least one pair of integers $(i, j)$, where $0 \leqq i$, $j \leqq p$ and $i \neq j$, then the elements of the set $\left\{\nu_{r}(\infty)\right\}_{r=0}^{p}$ can be determined.

Proof. We consider (4.10) with $a(x, t)=a(x)-\operatorname{tg}(x)$,

$$
\begin{aligned}
g(x) & =\left(h_{1} / 2-x\right)^{5} x^{2} g_{0}, & & 0 \leqq x \leqq h_{1} / 2 \\
& =\left(x-h_{1} / 2\right)^{5}\left(\left(h_{2}+h_{1}\right) / 2-x\right)^{5}\left(x-h_{1}\right)^{2} g_{1}, & & h_{1} / 2 \leqq x \leqq\left(h_{2}+h_{1}\right) / 2
\end{aligned}
$$

and for the intervals $\left(h_{r}+h_{r-1}\right) / 2 \leqq x \leqq\left(h_{r+1}+h_{r}\right) / 2, \quad r=2, \ldots,(p-1)$, and $\left(h_{p}+h_{p-1}\right) / 2 \leqq x \leqq 1$, let $g(x)$ be defined by (4.11) and (4.12); and with $g_{0}^{*}=\left(h_{1} / 2\right)^{5} g_{0}$, $g_{r}^{*}=\left(\left(h_{r+1}-h_{r}\right) / 2\right)^{5}\left(\left(h_{r}-h_{r-1}\right) / 2\right)^{5} g_{r}, r=1, \ldots,(p-1), g_{p}^{*}=\left(\left(h_{p}-h_{p-1}\right) / 2\right)^{5} g_{p}$, the positive constants $g_{r}^{*}, r=0, \ldots, p$, are chosen in the following way. If all the $b_{r}$, $r=0, \ldots, p$, are equal, then we choose the $g_{r}^{*}$ so that $g_{0}^{*}>g_{1}^{*}>\cdots>g_{p}^{*}>0$. If $b_{r(1,1)}=b_{r(1,2)}=\cdots=b_{r\left(1, j_{1}\right)}<b_{r(2,1)}=b_{r(2,2)}=\cdots=b_{r\left(2, j_{2}\right)}<\cdots<b_{r(l, 1)}=b_{r(l, 2)}=\cdots=$ $b_{r\left(l, j_{l}\right)}$, where $l>1, j_{i} \geqq 1$ for $i=1, \ldots, l, \sum_{i=1}^{l} j_{i}=p+1$, and $h_{r(i, 1)}<h_{r(i, 2)}<\cdots<h_{r\left(i, j_{i}\right)}$ if $j_{i}>1$ for $i=1, \ldots, l$, then the positive constants $g_{r}^{*}, r=0, \ldots, p$, are chosen so that $g_{r(1,1)}^{*}>g_{r(1,2)}^{*}>\cdots>g_{r\left(1, j_{1}\right)}^{*}$ and for $i=2, \ldots, l, g_{r\left(i-1, j_{i-1}\right)}^{*}>g_{r(i, 1)}^{*}>g_{r(i, 2)}^{*}>\cdots$ $>g_{r\left(i, j_{i}\right)}^{*}$.

Putting $b_{r}(t)=-\left(\frac{1}{2}\right) d^{2} a\left(h_{r}, t\right) / d x^{2}=b_{r}+t g_{r}^{*}, r=0, \ldots, p$, it is a simple matter to verify that if $0 \leqq i, j \leqq p$ and $i \neq j$, then $\left(b_{i}(t) / b_{j}(t)\right)$ is continuous, positive, and either strictly increasing or strictly decreasing for $t \geqq 0$.

Finally, let $R_{m}^{*}=\left\{x \mid x=((2 j+1) /(2 k+1))^{2}\right.$, where $j$ and $k$ are integers, $j=0$, $\ldots, k$, and $k=0, \ldots,(2 m+4)\}$ and choose the positive number $t_{1}$ (see Theorem 4.4) so that if $0 \leqq i, j \leqq p$ and $i \neq j$, then $\left(b_{i}(t) / b_{j}(t)\right) \notin R_{m}^{*}$ for $0<t \leqq t_{1}$. 
We now assert that if we put $a_{r}=b_{r}\left(t_{1}\right), r=0, \ldots, p$, in subsection III.2 of Appendix III, and with $f_{r}(n)$ given by equation (III.2.1), solve for $\left(r^{\dagger}, n^{\dagger}\right)$ the equation $f_{r}(\eta)=m$ (see Theorem III.2.2) then $\nu_{0}(\infty)=2 n^{\dagger}+1$ if $r^{\dagger}=0, v_{r}+(\infty)=n^{\dagger}$ if $r^{\dagger} \neq 0$. Hence from (5.1.2) we can determine the elements $v_{r}(\infty), r=0, \ldots, p$. This assertion follows by arguing as in Theorem 4.4 .

THEOREM 5.1.5. Let $\left(b_{i} / b_{j}\right) \in R$ for at least one pair of integers $(i, j)$, where $0 \leqq i$, $j \leqq p$ and $i \neq j$. From the set of integers $\{r\}_{r=0}^{p}$, let the subset $X$ be formed in the following way: (1) if $1 \leqq r \leqq p$, then $r \in X$ iff $\nu_{r}(\infty)$ is an integer, and (2) $0 \in X$ iff $\nu_{0}(\infty)$ is an odd integer. Then $X$ contains at least one element, and moreover, if $X$ contains precisely one element, and if we denote this element by $r *$ and

(1) put $\nu_{0}(\infty)=2 n^{*}+1$ if $r^{*}=0$, then (1.3) is valid with $A_{1, m}=G_{2,1}\left(0, n^{*}\right), A_{2, m}$ $=G_{2,2}\left(0, n^{*}\right)$, and $A_{3, m}=G_{2,3}\left(0, n^{*}\right)$;

(2) put $\nu_{r^{*}}(\infty)=n^{*}$ if $r^{*} \neq 0$, then (1.3) is valid with $A_{1, m}=G_{1,1}\left(r^{*}, n^{*}\right), A_{2, m}=0$, and $A_{3, m}=G_{1,2}\left(r^{*}, n^{*}\right)$.

Proof. We observe from our previous remarks that $X$ contains at least one element. Referring to the proof of Theorem 5.1.4, we see that if $X$ contains precisely one element, then it must be $r^{\dagger}$, and (1) if $r^{\dagger}=0$, then $\nu_{0}(\infty)=2 n^{\dagger}+1$, and no element of the set $\left\{\nu_{r}(\infty)\right\}_{r=1}^{p}$ is an integer, while (2) if $r^{\dagger} \neq 0$, then $\nu_{r}+(\infty)=n^{\dagger}$ and $\nu_{0}(\infty)$ is not an odd integer. Our theorem now follows immediately from (5.1.1) if we put $r^{*}=r^{\dagger}$ and $n^{*}=n^{\dagger}$.

From now on in this section we shall always assume that $\left(b_{i} / b_{j}\right) \in R$ for at least one pair of integers $(i, j)$, where $0 \leqq i, j \leqq p$ and $i \neq j$. Then in order to extend Theorem 5.1.5 to the case where $X$ contains more than one element, we can no longer restrict ourselves to the investigation of the asymptotic behaviour of just $\lambda_{m}(\mu)$. Hence for nonnegative integer $N \neq m$ and $\mu>0$ put

$$
\begin{aligned}
\Lambda_{N}(\mu)=\left(\lambda_{N}(\mu)+\mu A\right) / \mu^{1 / 2}, \quad \nu_{r}(\mu, N)=\left\{\left(\Lambda_{N}(\mu) / 2\left(b_{r}\right)^{1 / 2}\right)\right. & \left.-\frac{1}{2}\right\}, \\
r & =0, \ldots, p .
\end{aligned}
$$

Now it is clear that we may argue with $\lambda_{N}(\mu)$ in precisely the same way as we did with $\lambda_{m}(\mu)$, and by modifying (5.1.1) and Theorem 5.1.2 so as to apply to $\lambda_{N}(\mu)$, we then know that $\nu_{r}(\mu, N)$ tends to a finite limit as $\mu \rightarrow \infty, r=0, \ldots, p$, and at least one such limit is an integer. We shall from now on denote $\lim _{\mu \rightarrow \infty} \nu_{r}(\mu, N)$ by $\nu_{r}(\infty, N), r=0, \ldots, p$; and by modifying the proof of Theorem 5.1.4 so as to apply to $\lambda_{N}(\mu)$ instead of $\lambda_{m}(\mu)$, we can determine the set $\left\{\nu_{r}(\infty, N)\right\}_{r=0}^{p}$.

Referring to Theorem 5.1.5, consider now the case where $X$ contains at least two elements. Denote the elements of $X$ by $\left\{r_{j}\right\}_{j=0}^{p^{*}}$, where $1 \leqq p^{*} \leqq p$, and for the moment assume that the elements of $X$ have been labelled in some definite manner. Then for $\mu>0$ we introduce the functions $Z_{j}(\mu), j=0, \ldots, p^{*}$, in the following way: (1) if $1 \leqq r_{j} \leqq p$, put $\nu_{r_{j}}(\infty)=n_{j}$ and $Z_{j}(\mu)=G_{1,2}\left(r_{j}, n_{j}\right)$, and (2) if $r_{j}=0$, put $\nu_{0}(\infty)$ 
$=2 n_{j}+1$ and $Z_{j}(\mu)=\mu^{1 / 4} G_{2,2}\left(0, n_{j}\right)+G_{2,3}\left(0, n_{j}\right)$. Then by relabelling if necessary, we shall from now on assume that for all $\mu$ sufficiently large

$$
Z_{0}(\mu) \leqq Z_{1}(\mu) \leqq \cdots \leqq Z_{p^{*}}(\mu),
$$

where if $Z_{i-1}(\mu)=Z_{i}(\mu)$ for $1 \leqq i \leqq p^{*}$ and all $\mu$ sufficiently large, then $h_{r_{i-1}}<h_{r_{i}}$.

THEOREM 5.1.6. Under the hypothesis of Theorem 5.1.5 assume that $X$ contains at least two elements. Let the elements of $X$ be denoted by $\left\{r_{j}\right\}_{j=0}^{p^{*}}$, where $1 \leqq p^{*} \leqq p$, and for $j=0, \ldots, p^{*}$ put (1) $\nu_{r_{j}}(\infty)=n_{j}$ if $r_{j} \neq 0$, and (2) $\nu_{0}(\infty)=2 n_{j}+1$ if $r_{j}=0$; here the $r_{j}, j=0, \ldots, p^{*}$, are arranged so that for all $\mu$ sufficiently large (5.1.5) is satisfied. Let $s=m-N_{1}$, where if $m=0$, or if $m>0$ and $\nu_{0}(\infty, m-1)<\nu_{0}(\infty)$, then $N_{1}=m$, while if $m>0$ and $\nu_{0}(\infty, m-1)=\nu_{0}(\infty)$, then $N_{1}$ is the smallest integer satisfying $\nu_{0}\left(\infty, N_{1}\right)=\nu_{0}(\infty)$. The $0 \leqq s \leqq p^{*}$, and

(1) if $r_{s}=0$, then (1.3) is valid with $A_{1, m}=G_{2,1}\left(0, n_{s}\right), A_{2, m}=G_{2,2}\left(0, n_{s}\right)$, and $A_{3, m}=G_{2,3}\left(0, n_{s}\right)$;

(2) if $r_{s} \neq 0$, then (1.3) is valid with $A_{1, m}=G_{1,1}\left(r_{s}, n_{s}\right), A_{2, m}=0$, and $A_{3, m}=$ $G_{1,2}\left(r_{s}, n_{s}\right)$.

5.2. The case $\alpha \neq 0$. For this case the analogue of (5.1.1) is

$$
K_{0}^{+}(\mu) \prod_{r=1}^{p} K_{r}(\mu)=o\left(\exp \left\{-\Delta \mu^{1 / 2}\right\}\right) \quad \text { as } \mu \rightarrow \infty
$$

where

$$
\begin{aligned}
K_{0}^{\dagger}(\mu)= & \cos \pi\left(\nu_{0}+1\right) / 2 \\
& +\sin \pi\left(\nu_{0}+1\right) / 2\left[\left\langle c_{0} A_{2}\left(\nu_{0}\right) / 3+\left(\Gamma\left(\left(1+\nu_{0}\right) / 2\right) / \Gamma\left(1+\nu_{0} / 2\right)\right)\left(\left(b_{0}\right)^{-1 / 4} / 2\right) \cot \alpha\right\rangle \mu^{-1 / 4}\right. \\
& \left.+\left\langle W_{0}\left(\nu_{0}\right)+f_{0}^{\dagger}(\mu)\right\rangle\left(\mu^{-1 / 2} / 2\right)\right],
\end{aligned}
$$

$f_{0}^{\dagger}(\mu)=o(1)$ as $\mu \rightarrow \infty$, and the remaining terms are defined in subsection 5.1.

THEOREM 5.2.1. If $p=0$, then (1.3) is valid with $A_{1, m}=G_{3,1}(0, m), A_{2, m}=G_{3,2}(0, m, \alpha)$, and $A_{3, m}=G_{3,3}(0, m, \alpha)$.

From now on in this section we shall always assume that $p>0$.

THEOREM 5.2.2. As $\mu \rightarrow \infty, \nu_{r}$ tends to a finite limit, $r=0, \ldots, p$, and at least one such limit is an integer.

In the sequel we shall denote $\lim _{\mu \rightarrow \infty} \nu_{r}$ by $\nu_{r}(\infty), r=0, \ldots, p$; and we observe from $\S 2$ that if $v_{r}(\infty)$ is an integer, then it must be a nonnegative integer. Moreover, we see from (5.2.1) that if $\nu_{0}(\infty)$ is not an even integer, then at least one element of the set $\left\{\nu_{r}(\infty)\right\}_{r=1}^{p}$ is an integer. As before, we also have

$$
\left(b_{0}\right)^{1 / 2}\left(\nu_{0}(\infty)+\frac{1}{2}\right)=\left(b_{r}\right)^{1 / 2}\left(\nu_{r}(\infty)+\frac{1}{2}\right), \quad r=0, \ldots, p .
$$

THEOREM 5.2.3. If $\left(b_{i} / b_{j}\right) \notin R$ for $i, j=0, \ldots, p$ and $i \neq j$, then the elements of the set $\left\{\nu_{r}(\infty)\right\}_{r=1}^{p}$ can be determined. Furthermore, precisely one element of this set is an 
integer, and $\nu_{0}(\infty)$ is either an even integer or nonintegral. If we denote by $\nu_{r^{*}}(\infty)$ the element which is an integer and

(1) put $\nu_{0}(\infty)=2 n^{*}$ if $r^{*}=0$, then (1.3) is valid with $A_{1, m}=G_{3,1}\left(0, n^{*}\right), A_{2, m}$ $=G_{3,2}\left(0, n^{*}, \alpha\right)$, and $A_{3, m}=G_{3,3}\left(0, n^{*}, \alpha\right)$;

(2) put $v_{r^{*}}(\infty)=n^{*}$ if $r^{*} \neq 0$, then (1.3) is valid with $A_{1, m}=G_{1,1}\left(r^{*}, n^{*}\right), A_{2, m}=0$, and $A_{3, m}=G_{1,2}\left(r^{*}, n^{*}\right)$.

Proof. First we observe from (5.2.2) that only one element of the set $\left\{\nu_{r}(\infty)\right\}_{r=0}^{p}$ can be an integer; and from our above remarks we also see that if $\nu_{0}(\infty)$ is integral, then it must be even. We now assert that in order to determine the tuple $\left(r^{*}, n^{*}\right)$ stated in our theorem, we need only put $a_{r}=b_{r}, r=0, \ldots, p$, in subsection III.2 of Appendix III, and with $f_{r}(n)$ given by equation (III.2.2), solve for $\left(r^{\dagger}, n^{\dagger}\right)$ the equation $f_{r}(n)=m$ (see Theorem III.2.1), and then we must have $r^{*}=r^{\dagger}, n^{*}=n^{\dagger}$. Our theorem then follows from (5.2.1) and (5.2.2).

THEOREM 5.2.4. If $\left(b_{i} / b_{j}\right) \in R$ for at least one pair of integers $(i, j)$, where $0 \leqq i$, $j \leqq p$ and $i \neq j$, then the elements of the set $\left\{\nu_{r}(\infty)\right\}_{r=0}^{p}$ can be determined.

Proof. With all terms as defined in the proof of Theorem 5.1.4, we now assert that if we put $a_{r}=b_{r}\left(t_{1}\right), r=0, \ldots, p$, in subsection III.2 of Appendix III, and with $f_{r}(n)$ given by equation (III.2.2), solve for $\left(r^{\dagger}, n^{\dagger}\right)$ the equation $f_{r}(n)=m$ (see Theorem III.2.2), then $\nu_{0}(\infty)=2 n^{\dagger}$ if $r^{\dagger}=0, \nu_{r^{\dagger}}(\infty)=n^{\dagger}$ if $r^{\dagger} \neq 0$. Our theorem now follows from (5.2.2).

THEOREM 5.2.5. Let $\left(b_{i} / b_{j}\right) \in R$ for at least one pair of integers $(i, j)$, where $0 \leqq i$, $j \leqq p$ and $i \neq j$. From the set of integers $\{r\}_{r=0}^{p}$, let the subset $X$ be formed in the following way: (1) if $1 \leqq r \leqq p$, then $r \in X$ iff $\nu_{r}(\infty)$ is an integer, and (2) $0 \in X$ iff $\nu_{0}(\infty)$ is an even integer. Then $X$ contains at least one element, and moreover, if $X$ contains precisely one element, and if we denote this element by $r^{*}$ and

(1) put $\nu_{0}(\infty)=2 n^{*}$ if $r^{*}=0$, then (1.3) is valid with $A_{1, m}=G_{3,1}\left(0, n^{*}\right), A_{2, m}$ $=G_{3,2}\left(0, n^{*}, \alpha\right)$, and $A_{3, m}=G_{3,3}\left(0, n^{*}, \alpha\right)$;

(2) put $\nu_{r^{*}}(\infty)=n^{*}$ if $r^{*} \neq 0$, then (1.3) is valid with $A_{1, m}=G_{1,1}\left(r^{*}, n^{*}\right), A_{2, m}=0$, and $A_{3, m}=G_{1,2}\left(r^{*}, n^{*}\right)$.

Proof. We observe from our previous remarks that $X$ contains at least one element. Referring to the proof of Theorem 5.2.4, we see that if $X$ contains precisely one element, then it must be $r^{\dagger}$, and (1) if $r^{\dagger}=0$, then $\nu_{0}(\infty)=2 n^{\dagger}$ and no element of the set $\left\{\nu_{r}(\infty)\right\}_{r=1}^{p}$ is an integer, while (2) if $r^{\dagger} \neq 0$, then $\nu_{r}+(\infty)=n^{\dagger}$ and $\nu_{0}(\infty)$ is not an even integer. Our theorem now follows directly from (5.2.1) if we put $r^{*}=r^{\dagger}$ and $n^{*}=n^{\dagger}$.

From now on in this section we shall assume that $\left(b_{i} / b_{j}\right) \in R$ for at least one pair of integers $(i, j)$, where $0 \leqq i, j \leqq p$ and $i \neq j$. For nonnegative integer $N \neq m$, we now consider $\lambda_{N}(\mu)$ and define $\Lambda_{N}(\mu)$ and $\nu_{r}(\mu, N), r=0, \ldots, p$, as in (5.1.4). Then we can argue with $\lambda_{N}(\mu)$ in precisely the same way as we did with $\lambda_{m}(\mu)$ in Theorem 5.2.2 to show that $\nu_{r}(\mu, N)$ tends to a finite limit as $\mu \rightarrow \infty, r=0, \ldots, p$, and at 
least one such limit is an integer. We shall from now on denote $\lim _{\mu \rightarrow \infty} \nu_{r}(\mu, N)$ by $\nu_{r}(\infty, N), r=0, \ldots, p$; and by modifying the proof of Theorem 5.2 .4 so as to apply to $\lambda_{N}(\mu)$ instead of $\lambda_{m}(\mu)$, we can determine the set $\left\{\nu_{r}(\infty, N)\right\}_{r=0}^{p}$.

Referring to Theorem 5.2.5, consider now the case where $X$ contains at least two elements. Denote the elements of $X$ by $\left\{r_{j}\right\}_{j=0}^{p^{*}}$, where $1 \leqq p^{*} \leqq p$, and for the moment assume that the elements of $X$ have been labelled in some definite manner. Then for $\mu>0$ we introduce the functions $Z_{j}(\mu), j=0, \ldots, p^{*}$, in the following way: (1) if $1 \leqq r_{j} \leqq p$, put $\nu_{r_{j}}(\infty)=n_{j}$ and $Z_{j}(\mu)=G_{1,2}\left(r_{j}, n_{j}\right)$, and (2) if $r_{j}=0$, put $\nu_{0}(\infty)=2 n_{j}$ and $Z_{j}(\mu)=\mu^{1 / 4} G_{3,2}\left(0, n_{j}, \alpha\right)+G_{3,3}\left(0, n_{j}, \alpha\right)$. Then by relabelling if necessary, we shall from now on assume that for all $\mu$ sufficiently large

$$
Z_{0}(\mu) \leqq Z_{1}(\mu) \leqq \cdots \leqq Z_{p} \cdot(\mu)
$$

where if $Z_{i-1}(\mu)=Z_{i}(\mu)$ for $1 \leqq i \leqq p^{*}$ and all $\mu$ sufficiently large, then $h_{r_{i-1}}<h_{r_{i}}$.

THEOREM 5.2.6. Under the hypothesis of Theorem 5.2.5 assume that $X$ contains at least two elements. Let the elements of $X$ be denoted by $\left\{r_{j}\right\}_{j=0}^{p^{*}}$, where $1 \leqq p^{*} \leqq p$, and for $j=0, \ldots, p^{*}$ put $(1) \nu_{r_{j}}(\infty)=n_{j}$ if $r_{j} \neq 0$, and (2) $\nu_{0}(\infty)=2 n_{j}$ if $r_{j}=0$; here the $r_{j}, j=0, \ldots, p^{*}$, are arranged so that for all $\mu$ sufficiently large (5.2.3) is satisfied. Let $s=m-N_{1}$, where if $m=0$, or if $m>0$ and $\nu_{0}(\infty, m-1)<\nu_{0}(\infty)$, then $N_{1}=m$, while if $m>0$ and $\nu_{0}(\infty, m-1)=\nu_{0}(\infty)$, then $N_{1}$ is the smallest integer satisfying $\nu_{0}\left(\infty, N_{1}\right)=\nu_{0}(\infty)$. Then $0 \leqq s \leqq p^{*}$, and

(1) if $r_{s}=0$, then (1.3) is valid with $A_{1, m}=G_{3,1}\left(0, n_{s}\right), A_{2, m}=G_{3,2}\left(0, n_{s}, \alpha\right)$, and $A_{3, m}=G_{3,3}\left(0, n_{s}, \alpha\right)$;

(2) if $r_{s} \neq 0$, then (1.3) is valid with $A_{1, m}=G_{1,1}\left(r_{s}, n_{s}\right), A_{2, m}=0$, and $A_{3, m}=$ $G_{1,2}\left(r_{s}, n_{s}\right)$.

6. Main results for the case $a(0)=A$ and $a(1)=A$.

6.0. Introduction. In this section we consider the case where $a(x)=A$ at precisely the finite set of points $\left\{h_{r}\right\}_{r=0}^{p+1}$, where $p \geqq 0$ and $0=h_{0}<h_{1}<\cdots<h_{p+1}=1$. Then throughout this section, unless otherwise stated, we shall consider the integer $m$ fixed as before and use the results of $\S 3$ to verify (1.3). Since for the most part the arguments of $\$ 4$ can be modified so as to apply to the work of this section, we shall therefore contend ourselves here with mainly stating our results and only elaborating on points where the arguments of $\S 4$ are no longer valid. Finally, the four cases, (1) $\alpha=0, \beta=\pi$, (2) $\alpha=0, \beta \neq \pi$, (3) $\alpha \neq 0, \beta \neq \pi$, and (4) $\alpha \neq 0, \beta=\pi$, will be treated separately; and for terminology we refer to $\S \S 2$ and 4 .

6.1. The case $\alpha=0, \beta=\pi$. An argument analogous to that used in Theorem 4.2, together with the results of $\S 3$, show that if $p>0$ (and with obvious modifications if $p=0$ ) then

$$
K_{0}^{*}(\mu) K_{p+1}^{*}(\mu) \prod_{r=1}^{p} K_{r}(\mu)=o\left(\exp \left\{-\Delta \mu^{1 / 2}\right\}\right) \quad \text { as } \mu \rightarrow \infty,
$$


where

$$
\begin{aligned}
K_{p+1}^{*}(\mu)= & \sin \pi\left(\nu_{p+1}+1\right) / 2 \\
& +\cos \pi\left(\nu_{p+1}+1\right) / 2\left[c_{p+1} A_{2}\left(\nu_{p+1}\right)\left(\mu^{-1 / 4} / 3\right)-\left(W_{p+1}\left(\nu_{p+1}\right)+f_{p+1}^{*}(\mu)\right) \mu^{-1 / 2} / 2\right],
\end{aligned}
$$

$f_{p+1}^{*}(\mu)=o(1)$ as $\mu \rightarrow \infty, W_{p+1}(x)$ as given by (4.4) with $r=p+1$, and all other terms are defined in subsection 5.1.

Theorem 6.1.1. As $\mu \rightarrow \infty, \nu_{r}$ tends to a finite limit, $r=0, \ldots,(p+1)$, and at least one such limit is an integer.

From now on in this section we shall denote $\lim _{\mu \rightarrow \infty} \nu_{r}$ by $\nu_{r}(\infty), r=0, \ldots,(p+1)$ and we observe from $\S 2$ that if $\nu_{r}(\infty)$ is an integer, then it must be a nonnegative integer. Moreover, we see from (6.1.1) that if $p=0$ and $\nu_{0}(\infty)$ is not an odd integer, then $\nu_{p+1}(\infty)$ must be an odd integer; while if $p>0$ and both $\nu_{0}(\infty)$ and $\nu_{p+1}(\infty)$ are not odd integers, then at least one element of the set $\left\{\nu_{r}(\infty)\right\}_{r=1}^{p}$ is an integer. Also from $\S 2$ and Theorem 6.1.1 we see that

$$
\left(b_{0}\right)^{1 / 2}\left(\nu_{0}(\infty)+\frac{1}{2}\right)=\left(b_{r}\right)^{1 / 2}\left(\nu_{r}(\infty)+\frac{1}{2}\right), \quad r=0, \ldots,(p+1) .
$$

THEOREM 6.1.2. If $\left(b_{i} / b_{j}\right) \notin R$ for $i, j=0, \ldots,(p+1)$ and $i \neq j$, then the elements of the set $\left\{\nu_{r}(\infty)\right\}_{r=0}^{p+1}$ can be determined. Furthermore, precisely one element of this set is an integer, and $\nu_{0}(\infty)$ is either an odd integer or nonintegral, while $\nu_{p+1}(\infty)$ is either an odd integer or nonintegral. If we denote by $\nu_{r^{*}}(\infty)$ the element which is an integer and

(1) put $\nu_{0}(\infty)=2 n^{*}+1$ if $r^{*}=0$, then (1.3) is valid with $A_{1, m}=G_{2,1}\left(0, n^{*}\right)$, $A_{2, m}=G_{2,2}\left(0, n^{*}\right)$, and $A_{3, m}=G_{2,3}\left(0, n^{*}\right)$;

(2) put $\nu_{p+1}(\infty)=2 n^{*}+1$ if $r^{*}=p+1$, then (1.3) is valid with $A_{1, m}=G_{2,1}\left(p+1, n^{*}\right)$, $A_{2, m}=-G_{2,2}\left(p+1, n^{*}\right)$, and $A_{3, m}=G_{2,3}\left(p+1, n^{*}\right)$;

(3) put $\nu_{r^{*}}(\infty)=n^{*}$ if $p>0$ and $1 \leqq r^{*} \leqq p$, then (1.3) is valid with $A_{1, m}=G_{1,1}\left(r^{*}, n^{*}\right)$, $A_{2, m}=0$, and $A_{3, m}=G_{1,2}\left(r^{*}, n^{*}\right)$.

Proof. First we observe from (6.1.2) that only one element of the set $\left\{\nu_{r}(\infty)\right\}_{r=0}^{p+1}$ can be an integer; and from our above remarks we also see that if $\nu_{0}(\infty)$ is integral, then it must be odd, while if $\nu_{p+1}(\infty)$ is integral, then it must also be odd. We now assert that in order to determine the tuple $\left(r^{*}, n^{*}\right)$ stated in our theorem, we need only put $a_{r}=b_{r}, r=0, \ldots,(p+1)$, in subsection III.3 of Appendix III, and with $f_{r}(n)$ given by equation (III.3.1), solve for $\left(r^{\dagger}, n^{\dagger}\right)$ the equation $f_{r}(n)=m$ (see Theorem III.3.1) and then we must have $r^{*}=r^{\dagger}, n^{*}=n^{\dagger}$. This assertion is proved by arguing with (6.1.1) and (6.1.2) as in the proof of Theorem 4.3. Our theorem then follows from (6.1.1) and (6.1.2).

THEOREM 6.1.3. If $\left(b_{i} / b_{j}\right) \in R$ for at least one pair of integers $(i, j)$, where $0 \leqq i$, $j \leqq p+1$ and $i \neq j$, then the elements of the set $\left\{\nu_{r}(\infty)\right\}_{r=0}^{p+1}$ can be determined. 
Proof. We consider (4.10) with $a(x, t)=a(x)-\operatorname{tg}(x)$,

$$
\begin{aligned}
g(x)= & \left(x-\left(h_{p}+h_{p-1}\right) / 2\right)^{5}\left(\left(1+h_{p}\right) / 2-x\right)^{5}\left(x-h_{p}\right)^{2} g_{p}, \\
= & \left(x-\left(1+h_{p}\right) / 2\right)^{5}(x-1)^{2} g_{p+1}, \quad\left(1+h_{p}\right) / 2 \leqq \\
& x \leqq 1,
\end{aligned}
$$

and for the intervals $0 \leqq x \leqq h_{1} / 2,\left(h_{r}+h_{r-1}\right) / 2 \leqq x \leqq\left(h_{r+1}+h_{r}\right) / 2, r=1, \ldots,(p-1)$, let $g(x)$ be defined as in equations (5.1.3) and (4.11); and with

$$
\begin{aligned}
g_{p+1}^{*} & =\left(\left(1-h_{p}\right) / 2\right)^{5} g_{p+1}, \\
g_{p}^{*} & =\left(\left(h_{p}-h_{p-1}\right) / 2\right)^{5}\left(\left(1-h_{p}\right) / 2\right)^{5} g_{p}, \\
g_{r}^{*} & =\left(\left(h_{r+1}-h_{r}\right) / 2\right)^{5}\left(\left(h_{r}-h_{r-1}\right) / 2\right)^{5} g_{r},
\end{aligned}
$$

$r=1, \ldots,(p-1), g_{0}^{*}=\left(h_{1} / 2\right)^{5} g_{0}$, the positive constants $g_{r}^{*}, r=0, \ldots,(p+1)$, are chosen in the following way. If all the $b_{r}, r=0, \ldots,(p+1)$, are equal, then we choose the $g_{r}^{*}$ so that $g_{0}^{*}>g_{1}^{*}>\cdots>g_{p+1}^{*}>0$. If $b_{r(1,1)}=b_{r(1,2)}=\cdots=b_{r\left(1, j_{1}\right)}<b_{r(2,1)}$ $=b_{r(2,2)}=\cdots=b_{r\left(2, j_{2}\right)}<\cdots<b_{r(l, 1)}=b_{r(l, 2)}=\cdots=b_{r\left(l, j_{l}\right)}$, where $l>1, \quad j_{i} \geqq 1$ for $i=1, \ldots, l, \sum_{i=1}^{l} j_{i}=p+2$, and $h_{r(i, 1)}<h_{r(i, 2)}<\cdots<h_{r\left(i, j_{i}\right)}$ if $j_{i}>1$ for $i=1, \ldots, l$, then the positive constants $g_{r}^{*}, r=0, \ldots,(p+1)$, are chosen so that $g_{r(1,1)}^{*}>g_{r(1,2)}^{*}$ $>\cdots>g_{r\left(1, j_{1}\right)}^{*}$, and for $i=2, \ldots, l, g_{r\left(i-1, j_{t-1}\right)}^{*}>g_{r(i, 1)}^{*}>g_{r(i, 2)}^{*}>\cdots>g_{r\left(i, j_{i}\right)}^{*}$.

Putting $b_{r}(t)=-\left(\frac{1}{2}\right) d^{2} a\left(h_{r}, t\right) / d x^{2}=b_{r}+t_{r}^{*}, r=0, \ldots,(p+1)$, it is a simple matter to verify that if $0 \leqq i, j \leqq p+1$ and $i \neq j$, then $\left(b_{i}(t) / b_{j}(t)\right)$ is continuous, positive, and either strictly increasing or strictly decreasing for $t \geqq 0$.

Finally, let $R_{m}^{*}=\left\{x \mid x=((2 j+1) /(2 k+1))^{2}\right.$, where $j$ and $k$ are integers, $j=0, \ldots, k$ and $k=0, \ldots,(2 m+4)\}$; and choose the positive number $t_{1}$ (see Theorem 4.4) so that if $0 \leqq i, j \leqq p+1$ and $i \neq j$, then $\left(b_{i}(t) / b_{j}(t)\right) \notin R_{m}^{*}$ for $0<t \leqq t_{1}$.

We now assert that if we put $a_{r}=b_{r}\left(t_{1}\right), r=0, \ldots,(p+1)$, in subsection III.3 of Appendix III, and with $f_{r}(n)$ given by equation (III.3.1), solve for $\left(r^{\dagger}, n^{\dagger}\right)$ the equation $f_{r}(n)=m$ (see Theorem III.3.2) then $\nu_{0}(\infty)=2 n^{\dagger}+1$ if $r^{\dagger}=0, \nu_{p+1}(\infty)$ $=2 n^{\dagger}+1$ if $r^{\dagger}=p+1$, and $\nu_{r}+(\infty)=n^{\dagger}$ if $p>0$ and $1 \leqq r^{\dagger} \leqq p$. Hence using (6.1.2) we can determine the elements $\nu_{r}(\infty), r=0, \ldots,(p+1)$. This assertion follows by arguing as in Theorem 4.4 .

THEOREM 6.1.4. Let $\left(b_{i} / b_{j}\right) \in R$ for at least one pair of integers $(i, j)$, where $0 \leqq i$, $j \leqq p+1$ and $i \neq j$. From the set of integers $\{r\}_{r=0}^{p+1}$, let the subset $X$ be formed in the following way: (1) if $p>0$ and $1 \leqq r \leqq p$, then $r \in X$ iff $\nu_{r}(\infty)$ is an integer, (2) $0 \in X$ iff $\nu_{0}(\infty)$ is an odd integer, and $(3)(p+1) \in X$ iff $\nu_{p+1}(\infty)$ is an odd integer. Then $X$ contains at least one element, and moreover, if $X$ contains precisely one element, and if we denote this element by $r^{*}$ and

(1) put $\nu_{0}(\infty)=2 n^{*}+1$ if $r^{*}=0$, then (1.3) is valid with $A_{1, m}=G_{2,1}\left(0, n^{*}\right)$, $A_{2, m}=G_{2,2}\left(0, n^{*}\right)$, and $A_{3, m}=G_{2,3}\left(0, n^{*}\right)$;

(2) put $v_{p+1}(\infty)=2 n^{*}+1$ if $r^{*}=p+1$, then (1.3) is valid with $A_{1, m}=G_{2,1}\left(p+1, n^{*}\right)$, $A_{2, m}=-G_{2,2}\left(p+1, n^{*}\right)$, and $A_{3, m}=G_{2,3}\left(p+1, n^{*}\right)$;

(3) put $v_{r^{*}}(\infty)=n^{*}$ if $p>0$ and $1 \leqq r^{*} \leqq p$, then (1.3) is valid with $A_{1, m}=G_{1,1}\left(r^{*}, n^{*}\right)$, $A_{2, m}=0$, and $A_{3, m}=G_{1,2}\left(r^{*}, n^{*}\right)$. 
Proof. We observe from our previous remarks that $X$ contains at least one element. Referring to the proof of Theorem 6.1.3, we see that if $X$ contains precisely one element, then it must be $r^{\dagger}$, and (1) if $r^{\dagger}=0$, then $\nu_{0}(\infty)=2 n^{\dagger}+1, v_{p+1}(\infty)$ is not an odd integer, and no element of the set $\left\{\nu_{r}(\infty)\right\}_{r=1}^{p}$ is an integer if $p>0$, (2) if $r^{\dagger}=p+1$, then $\nu_{p+1}(\infty)=2 n^{\dagger}+1, \nu_{0}(\infty)$ is not an odd integer, and no element of the set $\left\{\nu_{r}(\infty)\right\}_{r=1}^{p}$ is an integer if $p>0$, and (3) if $p>0$ and $1 \leqq r^{\dagger} \leqq p$, then $\nu_{r}+(\infty)=n^{\dagger}$ and both $\nu_{0}(\infty)$ and $\nu_{p+1}(\infty)$ are not odd integers. Our theorem now follows immediately from (6.1.1) if we put $r^{*}=r^{\dagger}$ and $n^{*}=n^{\dagger}$.

From now on in this section we shall always assume that $\left(b_{i} / b_{j}\right) \in R$ for at least one pair of integers $(i, j)$, where $0 \leqq i, j \leqq p+1$ and $i \neq j$. Then in order to extend Theorem 6.1.4 to the case where $X$ contains more than one element, we can no longer restrict ourselves to the investigation of the asymptotic behaviour of just $\lambda_{m}(\mu)$. Hence for nonnegative integer $N \neq m$ an $\triangleleft \mu>0$ put

$$
\begin{aligned}
& \Lambda_{N}(\mu)=\left(\lambda_{N}(\mu)+\mu A\right) / \mu^{1 / 2}, \quad \nu_{r}(\mu, N)=\left\{\left(\Lambda_{N}(\mu) / 2\left(b_{r}\right)^{1 / 2}\right)-\frac{1}{2}\right\}, \\
& r=0, \ldots,(p+1) .
\end{aligned}
$$

Now it is clear that we may argue with $\lambda_{N}(\mu)$ in precisely the same way as we did with $\lambda_{m}(\mu)$, and by modifying (6.1.1) and Theorem 6.1.1 so as to apply to $\lambda_{N}(\mu)$, we then know that $\nu_{r}(\mu, N)$ tends to a finite limit as $\mu \rightarrow \infty, r=0, \ldots,(p+1)$, and at least one such limit is an integer. We shall from now on denote $\lim _{\mu \rightarrow \infty} \nu_{r}(\mu, N)$ by $\nu_{r}(\infty, N), r=0, \ldots,(p+1)$; and by modifying the proof of Theorem 6.1 .3 so as to apply to $\lambda_{N}(\mu)$ instead of $\lambda_{m}(\mu)$, we can determine the set $\left\{\nu_{r}(\infty, N)\right\}_{r=0}^{p+1}$.

Referring to Theorem 6.1.4, consider now the case where $X$ contains at least two elements. Denote the elements of $X$ by $\left\{r_{j}\right\}_{j=0}^{p^{*}}$, where $1 \leqq p^{*} \leqq p+1$, and for the moment assume that the elements of $X$ have been labelled in some definite manner. Then for $\mu>0$ we introduce the functions $Z_{j}(\mu), j=0, \ldots, p^{*}$, in the following way: (1) if $p>0$ and $1 \leqq r_{j} \leqq p$, put $\nu_{r_{j}}(\infty)=n_{j}$ and $Z_{j}(\mu)=G_{1,2}\left(r_{j}, n_{j}\right)$, (2) if $r_{j}=0$, put $\nu_{0}(\infty)=2 n_{j}+1$ and $Z_{j}(\mu)=\mu^{1 / 4} G_{2,2}\left(0, n_{j}\right)+G_{2,3}\left(0, n_{j}\right)$, and (3) if $r_{j}=p+1$, put $\nu_{p+1}(\infty)=2 n_{j}+1$ and $Z_{j}(\mu)=-\mu^{1 / 4} G_{2,2}\left(p+1, n_{j}\right)+G_{2,3}\left(p+1, n_{j}\right)$. Then by relabelling if necessary, we shall from now on assume that for all $\mu$ sufficiently large

$$
Z_{0}(\mu) \leqq Z_{1}(\mu) \leqq \cdots \leqq Z_{p^{*}}(\mu)
$$

where if $Z_{i-1}(\mu)=Z_{i}(\mu)$ for $1 \leqq i \leqq p^{*}$ and all $\mu$ sufficiently large, then $h_{r_{i-1}}<h_{r_{i}}$.

THEOREM 6.1.5. Under the hypothesis of Theorem 6.1.4 assume that $X$ contains at least two elements. Let the elements of $X$ be denoted by $\left\{r_{j}\right\}_{j=0}^{* *}$, where $1 \leqq p^{*} \leqq p+1$, and for $j=0, \ldots, p^{*}$ put (1) $\nu_{r_{j}}(\infty)=n_{j}$ if $p>0$ and $1 \leqq r_{j} \leqq p,(2) \nu_{0}(\infty)=2 n_{j}+1$ if $r_{j}=0$, and (3) $\nu_{p+1}(\infty)=2 n_{j}+1$ if $r_{j}=p+1$; here the $r_{j}, j=0, \ldots, p^{*}$, are arranged so that for all $\mu$ sufficiently large (6.1.4) is satisfied. Let $s=m-N_{1}$, where if $m=0$, or if $m>0$ and $\nu_{0}(\infty, m-1)<\nu_{0}(\infty)$, then $N_{1}=m$, while if $m>0$ and $\nu_{0}(\infty, m-1)$ $=\nu_{0}(\infty)$, then $N_{1}$ is the smallest integer satisfying $\nu_{0}\left(\infty, N_{1}\right)=\nu_{0}(\infty)$. The $10 \leqq s \leqq p^{*}$, and

(1) if $r_{s}=0$, then (1.3) is valid with $A_{1, m}=G_{2,1}\left(0, n_{s}\right), A_{2, m}=G_{2,2}\left(0, n_{s}\right)$, and $A_{3, m}=G_{2,3}\left(0, n_{s}\right)$; 
(2) if $r_{s}=p+1$, then (1.3) is valid with $A_{1, m}=G_{2,1}\left(p+1, n_{s}\right), A_{2, m}=-G_{2,2}\left(p+1, n_{s}\right)$, and $A_{3, m}=G_{2,3}\left(p+1, n_{s}\right)$;

(3) if $p>0$ and $1 \leqq r_{s} \leqq p$, then (1.3) is valid with $A_{1, m}=G_{1,1}\left(r_{s}, n_{s}\right), A_{2, m}=0$, and $A_{3, m}=G_{1,2}\left(r_{s}, n_{s}\right)$.

6.2. The case $\alpha=0, \beta \neq \pi$. For this case the analogue of (6.1.1) is

$$
K_{0}^{*}(\mu) K_{p+1}^{\dagger}(\mu) \prod_{r=1}^{p} K_{r}(\mu)=o\left(\exp \left\{-\Delta \mu^{1 / 2}\right\}\right) \quad \text { as } \mu \rightarrow \infty
$$

where

$$
\begin{aligned}
K_{p+1}^{\dagger}(\mu)= & \cos \pi\left(\nu_{r+1}+1\right) / 2 \\
-\sin \pi\left(\nu_{r+1}+1\right) / 2\left[\left\langlec_{p+1} A_{2}\left(\nu_{p+1}\right)^{\prime} 3+\right.\right. & \left(\Gamma\left(\left(1+\nu_{p+1}\right) / 2\right) / \Gamma\left(1+\nu_{p+1} / 2\right)\right) \\
& \times\left(\left(b_{p+1}\right)^{-1 / 4} / 2\right) \cot \beta \mu^{-1 / 4} \\
& \left.-\left\langle W_{p+1}\left(\nu_{p+1}\right)+f_{p+1}^{\dagger}(\mu)\right\rangle\left(\mu^{-1 / 2} / 2\right)\right],
\end{aligned}
$$

$f_{p+1}^{+}(\mu)=o(1)$ as $\mu \rightarrow \infty$, and all other terms are as defined above.

THEOREM 6.2.1. As $\mu \rightarrow \infty, \nu_{r}$ tends to a finite limit, $r=0, \ldots,(p+1)$, and at least one such limit is an integer.

From now on in this section we shall denote $\lim _{\mu \rightarrow \infty} \nu_{r}$ by $\nu_{r}(\infty), r=0, \ldots,(p+1)$; and we observe from $\S 2$ that if $\nu_{r}(\infty)$ is an integer, then it must be a nonnegative integer. Moreover, we see from (6.2.1) that if $p=0$ and $\nu_{0}(\infty)$ is not an odd integer, then $\nu_{p+1}(\infty)$ must be an even integer; while if $p>0$, and $\nu_{0}(\infty)$ is not an odd integer, and $\nu_{p+1}(\infty)$ is not an even integer, then at least one element of the set $\left\{\nu_{r}(\infty)\right\}_{r=1}^{p}$ is an integer. As before, we also have

$$
\left(b_{0}\right)^{1 / 2}\left(\nu_{0}(\infty)+\frac{1}{2}\right)=\left(b_{r}\right)^{1 / 2}\left(\nu_{r}(\infty)+\frac{1}{2}\right), \quad r=0, \ldots,(p+1) .
$$

THEOREM 6.2.2. If $\left(b_{i} / b_{j}\right) \notin R$ for $i, j=0, \ldots,(p+1)$ and $i \neq j$, then the elements of the set $\left\{v_{r}(\infty)\right\}_{r=0}^{p+1}$ can be determined. Furthermore, precisely one element of this set is an integer, and $\nu_{0}(\infty)$ is either an odd integer or nonintegral, while $\nu_{p+1}(\infty)$ is either an even integer or nonintegral. If we denote by $\nu_{r^{*}}(\infty)$ the element which is an integer and

(1) put $\nu_{0}(\infty)=2 n^{*}+1$ if $r^{*}=0$, then (1.3) is valid with $A_{1, m}=G_{2,1}\left(0, n^{*}\right)$, $A_{2, m}=G_{2,2}\left(0, n^{*}\right)$, and $A_{3, m}=G_{2,3}\left(0, n^{*}\right)$;

(2) put $\nu_{p+1}(\infty)=2 n^{*}$ if $r^{*}=p+1$, then (1.3) is valid with $A_{1, m}=G_{3,1}\left(p+1, n^{*}\right)$, $A_{2, m}=-G_{3,2}\left(p+1, n^{*}, \beta\right)$, and $A_{3, m}=G_{3,3}\left(p+1, n^{*}, \beta\right)$;

(3) put $\nu_{r^{*}}(\infty)=n^{*}$ if $p>0$ and $1 \leqq r^{*} \leqq p$, then (1.3) is valid with $A_{1, m}=G_{1,1}\left(r^{*}, n^{*}\right)$, $A_{2, m}=0$, and $A_{3, m}=G_{1,2}\left(r^{*}, n^{*}\right)$.

Proof. First we observe from (6.2.2) that only one element of the set $\left\{\nu_{r}(\infty)\right\}_{r=0}^{p+1}$ can be an integer; and from the above remarks we also see that if $\nu_{0}(\infty)$ is integral, then it must be odd, while if $\nu_{p+1}(\infty)$ is integral, then it must be even. We now assert that in order to determine the tuple $\left(r^{*}, n^{*}\right)$ stated in our theorem, we need only put $a_{r}=b_{r}, r=0, \ldots,(p+1)$, in subsection III.3 of Appendix III, and with $f_{r}(n)$ 
given by equation (III.3.2), solve for $\left(r^{\dagger}, n^{\dagger}\right)$ the equation $f_{r}(n)=m$ (see Theorem III.3.1) and then we must have $r^{*}=r^{\dagger}, n^{*}=n^{\dagger}$. Our theorem then follows from (6.2.1) and (6.2.2).

THEOREM 6.2.3. If $\left(b_{i} / b_{j}\right) \in R$ for at least one pair of integers $(i, j)$, where $0 \leqq i$, $j \leqq p+1$ and $i \neq j$, then the elements of the set $\left\{\nu_{r}(\infty)\right\}_{r=0}^{p+1}$ can be determined.

Proof. With all terms as defined in the proof of Theorem 6.1.3, we now assert that if we put $a_{r}=b_{r}\left(t_{1}\right), r=0, \ldots,(p+1)$, in subsection III.3 of Appendix III, and with $f_{r}(n)$ given by equation (III.3.2), solve for $\left(r^{\dagger}, n^{\dagger}\right)$ the equation $f_{r}(n)=m$ (see Theorem III.3.2), then $\nu_{0}(\infty)=2 n^{\dagger}+1$ if $r^{\dagger}=0, \nu_{p+1}(\infty)=2 n^{\dagger}$ if $r^{\dagger}=p+1$, and $\nu_{r}+(\infty)=n^{\dagger}$ if $p>0$ and $1 \leqq r^{\dagger} \leqq p$. Our theorem now follows from (6.2.2).

THEOREM 6.2.4. Let $\left(b_{i} / b_{j}\right) \in R$ for at least one pair of integers $(i, j)$, where $0 \leqq i$, $j \leqq p+1$ and $i \neq j$. From the set of integers $\{r\}_{r=0}^{p+1}$, let the subset $X$ be formed in the following way: (1) if $p>0$ and $1 \leqq r \leqq p$, then $r \in X$ iff $\nu_{r}(\infty)$ is an integer, (2) $0 \in X$ iff $\nu_{0}(\infty)$ is an odd integer, and $(3)(p+1) \in X$ iff $\nu_{p+1}(\infty)$ is an even integer. Then $X$ contains at least one element, and moreover, if $X$ contains precisely one element, and if we denote this element by $r *$ and

(1) put $\nu_{0}(\infty)=2 n^{*}+1$ if $r^{*}=0$, then (1.3) is valid with $A_{1, m}=G_{2,1}\left(0, n^{*}\right)$, $A_{2, m}=G_{2,2}\left(0, n^{*}\right)$, and $A_{3, m}=G_{2,3}\left(0, n^{*}\right)$;

(2) put $\nu_{p+1}(\infty)=2 n^{*}$ if $r^{*}=p+1$, then (1.3) is valid with $A_{1, m}=G_{3,1}\left(p+1, n^{*}\right)$, $A_{2, m}=-G_{3,2}\left(p+1, n^{*}, \beta\right)$, and $A_{3, m}=G_{3,3}\left(p+1, n^{*}, \beta\right)$;

(3) put $\nu_{r^{*}}(\infty)=n^{*}$ if $p>0$ and $1 \leqq r^{*} \leqq p$, then (1.3) is valid with $A_{1, m}=G_{1,1}\left(r^{*}, n^{*}\right)$, $A_{2, m}=0$, and $A_{3, m}=G_{1,2}\left(r^{*}, n^{*}\right)$.

Proof. We observe from our previous remarks that $X$ contains at least one element. Referring to the proof of Theorem 6.2.3, we see that if $X$ contains precisely one element, then it must be $r^{\dagger}$, and (1) if $r^{\dagger}=0$, then $\nu_{0}(\infty)=2 n^{\dagger}+1, \nu_{p+1}(\infty)$ is not an even integer, and no element of the set $\left\{\nu_{r}(\infty)\right\}_{r=1}^{p}$ is an integer if $p>0$, (2) if $r^{\dagger}=p+1$, then $\nu_{p+1}(\infty)=2 n^{\dagger}, v_{0}(\infty)$ is not an odd integer, and no element of the set $\left\{\nu_{r}(\infty)\right\}_{r=1}^{p}$ is an integer if $p>0$, and (3) if $p>0$ and $1 \leqq r^{\dagger} \leqq p$, then $\nu_{r^{\dagger}}(\infty)=n^{\dagger}$, $\nu_{0}(\infty)$ is not an odd integer, and $\nu_{p+1}(\infty)$ is not an even integer. Our theorem now follows directly from (6.2.1) if we put $r^{*}=r^{\dagger}$ and $n^{*}=n^{\dagger}$.

From now on in this section we shall always assume that $\left(b_{i} / b_{j}\right) \in R$ for at least one pair of integers $(i, j)$, where $0 \leqq i, j \leqq p+1$ and $i \neq j$. For nonnegative integer $N \neq m$, we now consider $\lambda_{N}(\mu)$ and define $\Lambda_{N}(\mu)$ and $\nu_{r}(\mu, N), r=0, \ldots,(p+1)$, as in (6.1.3). Then we can argue with $\lambda_{N}(\mu)$ in precisely the same way as we did with $\lambda_{m}(\mu)$ in Theorem 6.2.1 to show that $\nu_{r}(\mu, N)$ tends to a finite limit as $\mu \rightarrow \infty$, $r=0, \ldots, p$, and at least one such limit is an integer. We shall from now on denote $\lim _{\mu \rightarrow \infty} \nu_{r}(\mu, N)$ by $\nu_{r}(\infty, N), r=0, \ldots,(p+1)$; and by modifying the proof of Theorem (6.2.3) so as to apply to $\lambda_{N}(\mu)$ instead of $\lambda_{m}(\mu)$, we can determirie the set $\left\{\nu_{r}(\infty, N)\right\}_{r=0}^{p+1}$.

Referring to Theorem 6.2.4, consider now the case where $X$ contains at least two elements. Denote the elements of $X$ by $\left\{r_{j}\right\}_{j=0}^{p^{*}}$, where $1 \leqq p^{*} \leqq p+1$, and for the 
moment assume that the elements of $X$ have been labelled in some definite manner. Then for $\mu>0$ we introduce the functions $Z_{j}(\mu), j=0, \ldots, p^{*}$, in the following way: (1) if $p>0$ and $1 \leqq r_{j} \leqq p$, put $\nu_{r_{j}}(\infty)=n_{j}$ and $Z_{j}(\mu)=G_{1,2}\left(r_{j}, n_{j}\right)$, (2) if $r_{j}=0$, put $\nu_{0}(\infty)=2 n_{j}+1$ and $Z_{j}(\mu)=\mu^{1 / 4} G_{2,2}\left(0, n_{j}\right)+G_{2,3}\left(0, n_{j}\right)$, and (3) if $r_{j}=p+1$, put $\nu_{p+1}(\infty)=2 n_{j}$ and $Z_{j}(\mu)=-\mu^{1 / 4} G_{3,2}\left(p+1, n_{j}, \beta\right)+G_{3,3}\left(p+1, n_{j}, \beta\right)$. Then by relabelling if necessary, we shall from now on assume that for all $\mu$ sufficiently large

$$
Z_{0}(\mu) \leqq Z_{1}(\mu) \leqq \cdots \leqq Z_{p^{*}}(\mu),
$$

where if $Z_{i-1}(\mu)=Z_{i}(\mu)$ for $1 \leqq i \leqq p^{*}$ and all $\mu$ sufficiently large, then $h_{r_{i-1}}<h_{r_{i}}$.

THEOREM 6.2.5. Under the hypothesis of Theorem 6.2.4 assume that $X$ contains at least two elements. Let the elements of $X$ be denoted by $\left\{r_{j}\right\}_{j=0}^{p^{*}}$, where $1 \leqq p^{*} \leqq p+1$, and for $j=0, \ldots, p^{*}$ put (1) $\nu_{r_{j}}(\infty)=n_{j}$ if $p>0$ and $1 \leqq r_{j} \leqq p,(2) \nu_{0}(\infty)=2 n_{j}+1$ if $r_{j}=0$, and (3) $\nu_{p+1}(\infty)=2 n_{j}$ if $r_{j}=p+1$; here the $r_{j}, j=0, \ldots, p^{*}$, are arranged so that for all $\mu$ sufficiently large (6.2.3) is satisfied. Let $s=m-N_{1}$, where if $m=0$, or if $m>0$ and $\nu_{0}(\infty, m-1)<\nu_{0}(\infty)$, then $N_{1}=m$, while if $m>0$ and $\nu_{0}(\infty, m-1)=\nu_{0}(\infty)$, then $N_{1}$ is the smallest integer satisfying $\nu_{0}\left(\infty, N_{1}\right)=\nu_{0}(\infty)$. Then $0 \leqq s \leqq p^{*}$, and

(1) if $r_{s}=0$, then (1.3) is valid with $A_{1, m}=G_{2,1}\left(0, n_{s}\right), A_{2, m}=G_{2,2}\left(0, n_{s}\right)$, and $A_{3, m}=G_{2,3}\left(0, n_{s}\right)$;

(2) if $r_{s}=p+1$, then (1.3) is valid with $A_{1, m}=G_{3,1}\left(p+1, n_{s}\right), A_{2, m}=$ $-G_{3,2}\left(p+1, n_{s}, \beta\right)$, and $A_{3, m}=G_{3,3}\left(p+1, n_{s}, \beta\right)$;

(3) if $p>0$ and $1 \leqq r_{s} \leqq p$, then (1.3) is valid with $A_{1, m}=G_{1,1}\left(r_{s}, n_{s}\right), A_{2, m}=0$, and $A_{3, m}=G_{1,2}\left(r_{s}, n_{s}\right)$.

6.3. The case $\alpha \neq 0, \beta \neq \pi$. For this case the analogue of (6.1.1) is

$$
K_{0}^{\dagger}(\mu) K_{p+1}^{\dagger}(\mu) \prod_{r=0}^{p} K_{r}(\mu)=o\left(\exp \left\{-\Delta \mu^{1 / 2}\right\}\right) \quad \text { as } \mu \rightarrow \infty
$$

with all terminology as in subsection 6.2 above.

THEOREM 6.3.1. As $\mu \rightarrow \infty$, $\nu_{r}$ tends to a finite limit, $r=0, \ldots,(p+1)$, and at least one such limit is an integer.

From now on in this section we shall denote $\lim _{\mu \rightarrow \infty} \nu_{r}$ by $\nu_{r}(\infty), r=0, \ldots,(p+1)$; and we observe from $\S 2$ that if $\nu_{r}(\infty)$ is an integer, then it must be a nonnegative integer. Moreover, we see from (6.3.1) that if $p=0$ and $\nu_{0}(\infty)$ is not an even integer, then $\nu_{p+1}(\infty)$ must be an even integer; while if $p>0$ and both $\nu_{0}(\infty)$ and $\nu_{p+1}(\infty)$ are not even integers, then at least one element of the set $\left\{\nu_{r}(\infty)\right\}_{r=1}^{p}$ is an integer. As before, we also have

$$
\left(b_{0}\right)^{1 / 2}\left(\nu_{0}(\infty)+\frac{1}{2}\right)=\left(b_{r}\right)^{1 / 2}\left(\nu_{r}(\infty)+\frac{1}{2}\right), \quad r=0, \ldots,(p+1) .
$$

Theorem 6.3.2. If $\left(b_{i} / b_{j}\right) \notin R$ for $i, j=0, \ldots,(p+1)$ and $i \neq j$, then the elements of the set $\left\{\nu_{r}(\infty)\right\}_{r=0}^{p+1}$ can be determined. Furthermore, precisely one element of this set is an integer, and $\nu_{0}(\infty)$ is either an even integer or nonintegral, while $\nu_{p+1}(\infty)$ is 
either an even integer or nonintegral. If we denote by $\nu_{r^{*}}(\infty)$ the element which is an integer and

(1) put $\nu_{0}(\infty)=2 n^{*}$ if $r^{*}=0$, then (1.3) is valid with $A_{1, m}=G_{3,1}\left(0, n^{*}\right), A_{2, m}$ $=G_{3,2}\left(0, n^{*}, \alpha\right)$, and $A_{3, m}=G_{3,3}\left(0, n^{*}, \alpha\right)$;

(2) put $\nu_{p+1}(\infty)=2 n^{*}$ if $r^{*}=p+1$, then (1.3) is valid with $A_{1, m}=G_{3,1}\left(p+1, n^{*}\right)$, $A_{2, m}=-G_{3,2}\left(p+1, n^{*}, \beta\right)$, and $A_{3, n}=G_{3,3}\left(p+1, n^{*}, \beta\right)$;

(3) put $\nu_{r^{*}}(\infty)=n^{*}$ if $p>0$ and $1 \leqq r^{*} \leqq p$, then (1.3) is valid with $A_{1, m}=G_{1,1}\left(r^{*}, n^{*}\right)$, $A_{2, m}=0$, and $A_{3, m}=G_{1,2}\left(r^{*}, n^{*}\right)$.

Proof. First we observe from (6.3.2) that only one element of the set $\left\{\nu_{r}(\infty)\right\}_{r=0}^{p+1}$ can be an integer; and from our above remarks we also see that if $\nu_{0}(\infty)$ is integral, then it must be even, while if $\nu_{p+1}(\infty)$ is integral, then it must also be even. We now assert that in order to determine the tuple $\left(r^{*}, n^{*}\right)$ stated in our theorem, we need only put $a_{r}=b_{r}, r=0, \ldots,(p+1)$, in subsection III.3 of Appendix III, and with $f_{r}(n)$ given by equation (III.3.3), solve for $\left(r^{\dagger}, n^{\dagger}\right)$ the equation $f_{r}(n)=m$ (see Theorem III.3.1) and then we must have $r^{*}=r^{\dagger}, n^{*}=n^{\dagger}$. Our theorem now follows from (6.3.1) and (6.3.2).

THEOREM 6.3.3. If $\left(b_{i} / b_{j}\right) \in R$ for at least one pair of integers $(i, j)$, where $0 \leqq i$, $j \leqq p+1$ and $i \neq j$, then the elements of the set $\left\{\nu_{r}(\infty)\right\}_{r=0}^{p+1}$ can be determined.

Proof. With all terms as defined in the proof of Theorem 6.1.3, we now assert that if we put $a_{r}=b_{r}\left(t_{1}\right), r=0, \ldots,(p+1)$, in subsection III.3 of Appendix III, and with $f_{r}(n)$ given by equation (III.3.3), solve for $\left(r^{\dagger}, n^{\dagger}\right)$ the equation $f_{r}(n)=m$ (see Theorem III.3.2) then $\nu_{0}(\infty)=2 n^{\dagger}$ if $r^{\dagger}=0, \nu_{p+1}(\infty)=2 n^{\dagger}$ if $r^{\dagger}=p+1$, and $\nu_{r}+(\infty)=n^{\dagger}$ if $p>0$ and $1 \leqq r^{\dagger} \leqq p$. Our theorem now follows from (6.3.2).

THEOREM 6.3.4. Let $\left(b_{i} / b_{j}\right) \in R$ for at least one pair of integers $(i, j)$, where $0 \leqq i$, $j \leqq p+1$ and $i \neq j$. From the set of integers $\{r\}_{r=0}^{p+1}$, let the subset $X$ be formed in the following way: (1) if $p>0$ and $1 \leqq r \leqq p$, then $r \in X$ iff $\nu_{r}(\infty)$ is an integer, (2) $0 \in X$ iff $\nu_{0}(\infty)$ is an even integer, and $(3)(p+1) \in X$ iff $\nu_{p+1}(\infty)$ is an even integer. Then $X$ contains at least one element, and moreover, if $X$ contains precisely one element, and if we denote this element by $r^{*}$ and

(1) put $\nu_{0}(\infty)=2 n^{*}$ if $r^{*}=0$, then (1.3) is valid with $A_{1, m}=G_{3,1}\left(0, n^{*}\right), A_{2, m}$ $=G_{3,2}\left(0, n^{*}, \alpha\right)$, and $A_{3, m}=G_{3,3}\left(0, n^{*}, \alpha\right)$;

(2) put $\nu_{p+1}(\infty)=2 n^{*}$ if $r^{*}=p+1$, then (1.3) is valid with $A_{1, m}=G_{3,1}\left(p+1, n^{*}\right)$, $A_{2, m}=-G_{3,2}\left(p+1, n^{*}, \beta\right)$, and $A_{3, m}=G_{3,3}\left(p+1, n^{*}, \beta\right)$;

(3) put $\nu_{r^{*}}(\infty)=n^{*}$ if $p>0$ and $1 \leqq r^{*} \leqq p$, then (1.3) is valid with $A_{1, m}=G_{1,1}\left(r^{*}, n^{*}\right)$, $A_{2, m}=0$, and $A_{3, m}=G_{1,2}\left(r^{*}, n^{*}\right)$.

Proof. We observe from our previous remarks that $X$ contains at least one element. Referring to the proof of Theorem 6.3.3, we see that if $X$ contains precisely one element, then it must be $r^{\dagger}$, and (1) if $r^{\dagger}=0$, then $\nu_{0}(\infty)=2 n^{\dagger}, \nu_{p+1}(\infty)$ is not an even integer, and no element of the set $\left\{\nu_{r}(\infty)\right\}_{r=1}^{p}$ is an integer if $p>0$, (2) if $r^{\dagger}=p+1$, then $\nu_{p+1}(\infty)=2 n^{\dagger}, \nu_{0}(\infty)$ is not an even integer, and no element of the 
set $\left\{\nu_{r}(\infty)\right\}_{r=1}^{p}$ is an integer if $p>0$, and (3) if $p>0$ and $1 \leqq r^{\dagger} \leqq p$, then $\nu_{r}+(\infty)=n^{\dagger}$ and both $\nu_{0}(\infty)$ and $\nu_{p+1}(\infty)$ are not even integers. Our theorem now follows directly from (6.3.1) if we put $r^{*}=r^{\dagger}, n^{*}=n^{\dagger}$.

From now on in this section we shall always assume that $\left(b_{i} / b_{j}\right) \in R$ for at least one pair of integers $(i, j)$, where $0 \leqq i, j \leqq p+1$ and $i \neq j$. For nonnegative integer $N \neq m$, we now consider $\lambda_{N}(\mu)$ and define $\Lambda_{N}(\mu)$ and $\nu_{r}(\mu, N), r=0, \ldots,(p+1)$, as in (6.1.3). Then we can argue with $\lambda_{N}(\mu)$ in precisely the same way as we did with $\lambda_{m}(\mu)$ in Theorem 6.3.1 to show that $\nu_{r}(\mu, N)$ tends to a finite limit as $\mu \rightarrow \infty$, $r=0, \ldots,(p+1)$, and at least one such limit is an integer. We shall from now on denote $\lim _{\mu \rightarrow \infty} \nu_{r}(\mu, N)$ by $\nu_{r}(\infty, N), r=0, \ldots,(p+1)$; and by modifying the proof of Theorem 6.2.3 so as to apply to $\lambda_{N}(\mu)$ instead of $\lambda_{m}(\mu)$, we can determine the set $\left\{\nu_{r}(\infty, N)\right\}_{r=0}^{p+1}$.

Referring to Theorem 6.3 .4 , consider now the case where $X$ contains at least two elements. Denote the elements of $X$ by $\left\{r_{j}\right\}_{j=0}^{p^{*}}$, where $1 \leqq p^{*} \leqq p+1$, and for the moment assume that the elements of $X$ have been labelled in some definite manner. Then for $\mu>0$ we introduce the functions $Z_{j}(\mu), j=0, \ldots, p^{*}$, in the following way: (1) if $p>0$ and $1 \leqq r_{j} \leqq p$, put $\nu_{r_{j}}(\infty)=n_{j}$ and $Z_{j}(\mu)=G_{1,2}\left(r_{j}, n_{j}\right)$, (2) if $r_{j}=0$, put $\nu_{0}(\infty)=2 n_{j}$ and $Z_{j}(\mu)=\mu^{1 / 4} G_{3,2}\left(0, n_{j}, \alpha\right)+G_{3,3}\left(0, n_{j}, \alpha\right)$, and (3) if $r_{j}=p+1$, put $\nu_{p+1}(\infty)=2 n_{j}$ and $Z_{j}(\mu)=-\mu^{1 / 4} G_{3,2}\left(p+1, n_{j}, \beta\right)+G_{3,3}\left(p+1, n_{j}, \beta\right)$. Then by relabelling if necessary, we shall from now on assume that for all $\mu$ sufficiently large

$$
Z_{0}(\mu) \leqq Z_{1}(\mu) \leqq \cdots \leqq Z_{p^{*}}(\mu),
$$

where if $Z_{i-1}(\mu)=Z_{i}(\mu)$ for $1 \leqq i \leqq p^{*}$ and all $\mu$ sufficiently large, then $h_{r_{i-1}}<h_{r_{i}}$.

THEOREM 6.3.5. Under the hypothesis of Theorem 6.3.4 assume that $X$ contains at least two elements. Let the elements of $X$ be denoted by $\left\{r_{j}\right\}_{j=0}^{p^{*}}$, where $1 \leqq p^{*} \leqq p+1$, and for $j=0, \ldots, p^{*}$ put $(1) \nu_{r_{j}}(\infty)=n_{j}$ if $p>0$ and $1 \leqq r_{j} \leqq p,(2) \nu_{0}(\infty)=2 n_{j}$ if $r_{j}=0$, and (3) $\nu_{p+1}(\infty)=2 n_{j}$ if $r_{j}=p+1$; here the $r_{j}, j=0, \ldots, p^{*}$, are arranged so that for all $\mu$ sufficiently large (6.3.3) is satisfied. Let $s=m-N_{1}$, where if $m=0$, or if $m>0$ and $\nu_{0}(\infty, m-1)<\nu_{0}(\infty)$, then $N_{1}=m$, while if $m>0$ and $\nu_{0}(\infty, m-1)=\nu_{0}(\infty)$, then $N_{1}$ is the smallest integer satisfying $\nu_{0}\left(\infty, N_{1}\right)=\nu_{0}(\infty)$. Then $0 \leqq s \leqq p^{*}$, and

(1) if $r_{s}=0$, then (1.3) is valid with $A_{1, m}=G_{3,1}\left(0, n_{s}\right), A_{2, m}=G_{3,2}\left(0, n_{s}, \alpha\right)$, and $A_{3, m}=G_{3,3}\left(0, n_{s}, \alpha\right)$;

(2) if $r_{s}=p+1$, then (1.3) is valid with $A_{1, m}=G_{3,1}\left(p+1, n_{s}\right), A_{2, m}=$ $-G_{3,2}\left(p+1, n_{s}, \beta\right)$, and $A_{3, m}=G_{3,3}\left(p+1, n_{s}, \beta\right)$;

(3) if $p>0$ and $1 \leqq r_{s} \leqq p$, then (1.3) is valid with $A_{1, m}=G_{1,1}\left(r_{s}, n_{s}\right), A_{2, m}=0$, and $A_{3, m}=G_{1,2}\left(r_{s}, n_{s}\right)$.

6.4. The case $\alpha \neq 0, \beta=\pi$. For this case the analogue of (6.1.1) is

$$
K_{0}^{\dagger}(\mu) K_{p+1}^{*}(\mu) \prod_{r=1}^{p} K_{r}(\mu)=o\left(\exp \left\{-\Delta \mu^{1 / 2}\right\}\right) \quad \text { as } \mu \rightarrow \infty,
$$

with all terminology as above. 
THEOREM 6.4.1. As $\mu \rightarrow \infty$, $\nu_{r}$ tends to a finite limit, $r=0, \ldots,(p+1)$, and at least one such limit is an integer.

From now on in this section we shall denote $\lim _{\mu \rightarrow \infty} \nu_{r}$ by $\nu_{r}(\infty), r=0, \ldots,(p+1)$; and we observe from $\S 2$ that if $\nu_{r}(\infty)$ is an integer, then it must be a nonnegative integer. Moreover, we see from (6.4.1) that if $p=0$ and $\nu_{0}(\infty)$ is not an even integer, then $\nu_{p+1}(\infty)$ must be an odd integer; while if $p>0$, and $\nu_{0}(\infty)$ is not an even integer, and $\nu_{p+1}(\infty)$ is not an odd integer, then at least one element of the set $\left\{\nu_{r}(\infty)\right\}_{r=1}^{p}$ is an integer. As before, we also have

$$
\left(b_{0}\right)^{1 / 2}\left(\nu_{0}(\infty)+\frac{1}{2}\right)=\left(b_{r}\right)^{1 / 2}\left(\nu_{r}(\infty)+\frac{1}{2}\right), \quad r=0, \ldots,(p+1) .
$$

THEOREM 6.4.2. If $\left(b_{i} / b_{j}\right) \notin R$ for $i, j=0, \ldots,(p+1)$ and $i \neq j$, then the elements of the set $\left\{v_{r}(\infty)\right\}_{r=0}^{p+1}$ can be determined. Furthermore, precisely one element of this set is an integer, and $\nu_{0}(\infty)$ is either an even integer or nonintegral, while $\nu_{p+1}(\infty)$ is either an odd integer or nonintegral. If we denote by $\nu_{r^{*}}(\infty)$ the element which is an integer and

(1) put $\nu_{0}(\infty)=2 n^{*}$ if $r^{*}=0$, then (1.3) is valid with $A_{1, m}=G_{3,1}\left(0, n^{*}\right), A_{2, m}$ $=G_{3,2}\left(0, n^{*}, \alpha\right)$, and $A_{3, m}=G_{3,3}\left(0, n^{*}, \alpha\right)$;

(2) put $v_{p+1}(\infty)=2 n^{*}+1$ if $r^{*}=p+1$, then (1.3) is valid with $A_{1, m}=G_{2,1}\left(p+1, n^{*}\right)$, $A_{2, m}=-G_{2,2}\left(p+1, n^{*}\right)$, and $A_{3, m}=G_{2,3}\left(p+1, n^{*}\right)$;

(3) put $\nu_{r^{*}}(\infty)=n^{*}$ if $p>0$ and $1 \leqq r^{*} \leqq p$, then (1.3) is valid with $A_{1, m}=G_{1,1}\left(r^{*}, n^{*}\right)$, $A_{2, m}=0$, and $A_{3, m}=G_{1,2}\left(r^{*}, n^{*}\right)$.

Proof. First we observe from (6.4.2) that only one element of the set $\left\{\nu_{r}(\infty)\right\}_{r=0}^{p+1}$ can be an integer; and from the above remarks we see that if $\nu_{0}(\infty)$ is integral, then it must be even, while if $\nu_{p+1}(\infty)$ is integral, then it must be odd. We now assert that in order to determine the tuple $\left(r^{*}, n^{*}\right)$ stated in our theorem, we need only put $a_{0}=b_{p+1}, a_{p+1}=b_{0}$, and $a_{r}=b_{r}$ for $r=1, \ldots, p$ if $p>0$, in subsection III.3 of Appendix III, and with $f_{r}(n)$ given by equation (III.3.2), solve for $\left(r^{\dagger}, n^{\dagger}\right)$ the equation $f_{r}(n)=m$ (see Theorem III.3.1); and then we must have $n^{*}=n^{\dagger}, r^{*}=p+1$ if $r^{\dagger}=0, r^{*}=0$ if $r^{\dagger}=p+1$, and $r^{*}=r^{\dagger}$ if $p>0$ and $1 \leqq r^{\dagger} \leqq p$. Our theorem then follows from (6.4.1) and (6.4.2).

THEOREM 6.4.3. If $\left(b_{i} / b_{j}\right) \in R$ for at least one pair of integers $(i, j)$, where $0 \leqq i$, $j \leqq p+1$ and $i \neq j$, then the elements of the set $\left\{\nu_{r}(\infty)\right\}_{r=0}^{p+1}$ can be determined.

Proof. With all terms as defined in the proof of Theorem 6.1.3, we now assert that if in subsection III.3 of Appendix III we put $a_{0}=b_{p+1}\left(t_{1}\right), a_{p+1}=b_{0}\left(t_{1}\right)$, and $a_{r}=b_{r}\left(t_{1}\right)$ for $r=1, \ldots, p$ if $p>0$, and with $f_{r}(n)$ given by equation (III.3.2), solve for $\left(r^{\dagger}, n^{\dagger}\right)$ the equation $f_{r}(n)=m$, then $\nu_{0}(\infty)=2 n^{\dagger}$ if $r^{\dagger}=p+1, \nu_{p+1}(\infty)=2 n^{\dagger}+1$ if $r^{\dagger}=0$, and $\nu_{r}+(\infty)=n^{\dagger}$ if $p>0$ and $1 \leqq r^{\dagger} \leqq p$. Our theorem now follows from (6.4.2).

THEOREM 6.4.4. Let $\left(b_{i} / b_{j}\right) \in R$ for at least one pair of integers $(i, j)$, where $0 \leqq i$, $j \leqq p+1$ and $i \neq j$. From the set of integers $\{r\}_{r=0}^{p+1}$, let the subset $X$ be formed in the following way: (1) if $p>0$ and $1 \leqq r \leqq p$, then $r \in X$ iff $\nu_{r}(\infty)$ is an integer, (2) $0 \in X$ 
iff $\nu_{0}(\infty)$ is an even integer, and (3) $(p+1) \in X$ iff $\nu_{p+1}(\infty)$ is an odd integer. Then $X$ contains at least one element, and moreover, if $X$ contains precisely one element, and if we denote this element by $r^{*}$ and

(1) put $\nu_{0}(\infty)=2 n^{*}$ if $r^{*}=0$, then (1.3) is valid with $A_{1, m}=G_{3,1}\left(0, n^{*}\right), A_{2, m}$ $=G_{3,2}\left(0, n^{*}, \alpha\right)$, and $A_{3, m}=G_{3,3}\left(0, n^{*}, \alpha\right)$;

(2) put $v_{p+1}(\infty)=2 n^{*}+1$ if $r^{*}=p+1$, then (1.3) is valid with $A_{1, m}=G_{2,1}\left(p+1, n^{*}\right)$, $A_{2, m}=-G_{2,2}\left(p+1, n^{*}\right)$, and $A_{3, m}=G_{2,3}\left(p+1, n^{*}\right)$;

(3) put $\nu_{r^{*}}(\infty)=n^{*}$ if $p>0$ and $1 \leqq r^{*} \leqq p$, then (1.3) is valid with $A_{1, m}=G_{1,1}\left(r^{*}, n^{*}\right)$, $A_{2, m}=0$, and $A_{3, m}=G_{1,2}\left(r^{*}, n^{*}\right)$.

Proof. We observe from our previous remarks that $X$ contains at least one element. Referring to the proof of Theorem 6.4.3, we see that if $X$ contains precisely one element, then it must be 0 if $r^{\dagger}=p+1,(p+1)$ if $r^{\dagger}=0$, and $r^{\dagger}$ if $p>0$ and $1 \leqq r^{\dagger} \leqq p$. Putting $n^{*}=n^{\dagger}, r^{*}=0$ if $r^{\dagger}=p+1, r^{*}=p+1$ if $r^{\dagger}=0$, and $r^{*}=r^{\dagger}$ if $p>0$ and $1 \leqq r^{\dagger} \leqq p$, we see that (1) if $r^{*}=0$, then $\nu_{0}(\infty)=2 n^{*}, \nu_{p+1}(\infty)$ is not an odd integer, and no element of the set $\left\{\nu_{r}(\infty)\right\}_{r=1}^{p}$ is an integer if $p>0$, (2) if $r^{*}=p+1$, then $\nu_{p+1}(\infty)=2 n^{*}+1, \nu_{0}(\infty)$ is not an even integer, and no element of the set $\left\{\nu_{r}(\infty)\right\}_{r=1}^{p}$ is an integer if $p>0$, and (3) if $p>0$ and $1 \leqq r^{*} \leqq p$, then $\nu_{r^{*}}(\infty)=n^{*}, \nu_{0}(\infty)$ is not an even integer, and $\nu_{p+1}(\infty)$ is not an odd integer. Our theorem now follows directly from (6.4.1).

From now on in this section we shall always assume that $\left(b_{i} / b_{j}\right) \in R$ for at least one pair of integers $(i, j)$, where $0 \leqq i, j \leqq p+1$ and $i \neq j$. For nonnegative integer $N \neq m$, consider now $\lambda_{N}(\mu)$ and define $\Lambda_{N}(\mu)$ and $\nu_{r}(\mu, N), r=0, \ldots,(p+1)$, as in (6.1.3). Then we can argue with $\lambda_{N}(\mu)$ in precisely the same way as we did with $\lambda_{m}(\mu)$ in Theorem 6.4.1 to show that $\nu_{r}(\mu, N)$ tends to a finite limit as $\mu \rightarrow \infty$, $r=0, \ldots,(p+1)$, and at least one such limit is an integer. We shall from now on denote $\lim _{\mu \rightarrow \infty} \nu_{r}(\mu, N)$ by $\nu_{r}(\infty, N), r=0, \ldots,(p+1)$; and by modifying the proof of Theorem 6.2 .3 so as to apply to $\lambda_{N}(\mu)$ instead of $\lambda_{m}(\mu)$, we can determine the set $\left\{\nu_{r}(\infty, N)\right\}_{r=0}^{p+1}$.

Referring to Theorem 6.4 .4 , consider now the case where $X$ contains at least two elements. Denote the elements of $X$ by $\left\{r_{j}\right\}_{j=0}^{p^{*}}$, where $1 \leqq p^{*} \leqq p+1$, and for the moment assume that the elements of $X$ have been labelled in some definite manner. Then for $\mu>0$ we introduce the functions $Z_{j}(\mu), j=0, \ldots, p^{*}$, in the following way: (1) if $p>0$ and $1 \leqq r_{j} \leqq p$, put $\nu_{r_{j}}(\infty)=n_{j}$ and $Z_{j}(\mu)=G_{1,2}\left(r_{j}, n_{j}\right)$, (2) if $r_{j}=0$, put $\nu_{0}(\infty)=2 n_{j}$ and $Z_{j}(\mu)=\mu^{1 / 4} G_{3,2}\left(0, n_{j}, \alpha\right)+G_{3,3}\left(0, n_{j}, \alpha\right)$, and (3) if $r_{j}=p+1$, put $\nu_{p+1}(\infty)=2 n_{j}+1$ and $Z_{j}(\mu)=-\mu^{1 / 4} G_{2,2}\left(p+1, n_{j}\right)+G_{2,3}\left(p+1, n_{j}\right)$. Then by relabelling if necessary, we shall from now on assume that for all $\mu$ sufficiently large

$$
Z_{0}(\mu) \leqq Z_{1}(\mu) \leqq \cdots \leqq Z_{p^{*}}(\mu),
$$

where if $Z_{i-1}(\mu)=Z_{i}(\mu)$ for $1 \leqq i \leqq p^{*}$ and all $\mu$ sufficiently large, then $h_{r_{i-1}}<h_{r_{i}}$.

THEOREM 6.4.5. Under the hypothesis of Theorem 6.4.4 assume that $X$ contains at least two elements. Let the elements of $X$ be denoted by $\left\{r_{j}\right\}_{j=0}^{r^{*}}$, where $1 \leqq p^{*} \leqq p+1$, 
and for $j=0, \ldots, p^{*}$ put (1) $\nu_{r_{j}}(\infty)=n_{j}$ if $p>0$ and $1 \leqq r_{j} \leqq p$, (2) $\nu_{0}(\infty)=2 n_{j}$ if $r_{j}=0$, and (3) $\nu_{p+1}(\infty)=2 n_{j}+1$ if $r_{j}=p+1$; here the $r_{j}, j=0, \ldots, p^{*}$, are arranged so that for all $\mu$ sufficiently large (6.4.3) is satisfied. Let $s=m-N_{1}$, where if $m=0$, or if $m>0$ and $\nu_{0}(\infty, m-1)<\nu_{0}(\infty)$, then $N_{1}=m$, while if $m>0$ and $\nu_{0}(\infty, m-1)=\nu_{0}(\infty)$, then $N_{1}$ is the smallest integer satisfying $\nu_{0}\left(\infty, N_{1}\right)=\nu_{0}(\infty)$. Then $0 \leqq s \leqq p^{*}$, and

(1) if $r_{s}=0$, then (1.3) is valid with $A_{1, m}=G_{3,1}\left(0, n_{s}\right), A_{2, m}=G_{3,2}\left(0, n_{s}, \alpha\right)$, and $A_{3, m}=G_{3,3}\left(0, n_{s}, \alpha\right)$;

(2) if $r_{s}=p+1$, then (1.3) is valid with $A_{1, m}=G_{2,1}\left(p+1, n_{s}\right), A_{2, m}=$ $-G_{2,2}\left(p+1, n_{s}\right)$, and $A_{3, m}=G_{2,3}\left(p+1, n_{s}\right)$;

(3) if $p>0$ and $1 \leqq r_{s} \leqq p$, then (1.3) is valid with $A_{1, m}=G_{1,1}\left(r_{s}, n_{s}\right), A_{2, m}=0$, and $A_{3, m}=G_{1,2}\left(r_{s}, n_{s}\right)$.

Appendix I: Certain properties of parabolic cylinder functions. Here we shall consider Weber's equation [13, Chapter 16]

$$
y^{\prime \prime}(s)+\left(\nu+\frac{1}{2}-s^{2} / 4\right) y(s)=0, \quad,=d / d s,
$$

with the restriction that $-\infty<s<\infty,-\frac{1}{2} \leqq \nu \leqq C$, where $C$ is a positive constant. For a fundamental set of solutions we take $U_{1}(s, v)=D_{-v-1}(i s)$ and $U_{2}(s, v)$ $=D_{-v-1}(-i s)$, where $D_{v}(s)$ is the parabolic cylinder function; we observe that $U_{1}(0, \nu)=2^{-\nu / 2}(\pi / 2)^{1 / 2} / \Gamma(1+\nu / 2), \quad U_{1}^{\prime}(0, \nu)=-i 2^{-(\nu-1) / 2}(\pi / 2)^{1 / 2} / \Gamma((1+\nu) / 2), \quad$ and $U_{2}(0, v)=U_{1}(0, v), U_{2}^{\prime}(0, v)=$ the complex conjugate of $U_{1}^{\prime}(0, v)$, and hence $U_{2}(s, v)$ is the complex conjugate of $U_{1}(s, v),-\infty<s<\infty$. For $s \geqq 0$, we also introduce the solution

$$
U_{3}(s, \nu)=U_{1}(s, \nu) \int_{s}^{\infty} U_{1}^{-2}(t, \nu) d t=i e^{i \pi v / 2} D_{v}(s)
$$

From [13, Chapter 16], [14, Chapter 8], and using the methods of [10, Appendix I], we then have as $s \rightarrow \infty$, and uniformly in $\nu$,

$$
\begin{aligned}
& U_{1}(s, \nu)=s^{-v-1} \exp \left\{-i \pi(\nu+1) / 2+s^{2} / 4\right\}\left[1+(\nu+1)_{2} / 2 s^{2}+(\nu+1)_{4} / 8 s^{4}+O\left(s^{-6}\right)\right], \\
& U_{1}^{\prime}(s, \nu)=\left(s^{-\nu} / 2\right) \exp \left\{-i \pi(\nu+1) / 2+s^{2} / 4\right\} \\
& \times\left[1+(\nu-2)(\nu+1) / 2 s^{2}+(\nu-4)(\nu+1)_{3} / 8 s^{4}+O\left(s^{-6}\right)\right], \\
& U_{3}(s, \nu)=s^{v} \exp \left\{i \pi(\nu+1) / 2-s^{2} / 4\right\}\left[1-(-\nu)_{2} / 2 s^{2}+(-\nu)_{4} / 8 s^{4}+O\left(s^{-6}\right)\right], \\
& \int_{0}^{s} U_{1}^{2}(t, \nu) d t=s^{-2 v-3} \exp \left\{-i \pi(\nu+1)+s^{2} / 2\right\} \\
& \times\left[1+\left(\nu^{2}+5 \nu+5\right) / s^{2}+\left(\nu^{4}+12 \nu^{3}+54 \nu^{2}+101 \nu+64\right) / 2 s^{4}+O\left(s^{-6}\right)\right], \\
& \int_{0}^{s} U_{1}(t, v) U_{3}(t, v) d t=\log s+E_{1}(\nu)-i E_{2}(v)-(2 \nu+1) / 2 s^{2}-3\left(2 \nu^{2}+2 \nu+1\right) / 4 s^{4} \\
& -5\left(4 \nu^{3}+6 \nu^{2}+8 \nu+3\right) / 6 s^{6}+O\left(s^{-8}\right), \\
& \int_{0}^{s} t^{3} U_{1}^{2}(t, \nu) d t=s^{-2 v} \exp \left\{-i \pi(\nu+1)+s^{2} / 2\right\} \\
& \times\left[1+\left(\nu^{2}+5 \nu+2\right) / s^{2}+\left(\nu^{4}+12 \nu^{3}+48 \nu^{2}+59 \nu+22\right) / 2 s^{4}+O\left(s^{-6}\right)\right],
\end{aligned}
$$




$$
\begin{aligned}
& \int_{0}^{s} t^{3} U_{1}(t, v) U_{3}(t, v) d t=s^{3} / 3+(2 \nu+1) s-\left(A_{1}(v)-i A_{2}(v)\right) / 3 \\
& -3\left(2 \nu^{2}+2 \nu+1\right) / s-5\left(4 \nu^{3}+6 \nu^{2}+8 \nu+3\right) / 3 s^{3}+O\left(s^{-5}\right), \\
& \int_{0}^{s} t^{4} U_{1}^{2}(t, \nu) d t=s^{-2 v+1} \exp \left\{-i \pi(\nu+1)+s^{2} / 2\right\}\left[1+\left(\nu^{2}+5 \nu+1\right) / s^{2}+O\left(s^{-4}\right)\right], \\
& \int_{0}^{s} t^{4} U_{1}(t, \nu) U_{3}(t, \nu) d t \\
& =s^{4} / 4+(2 \nu+1) s^{2} / 2+3\left(2 \nu^{2}+2 \nu+1\right) \log s+B_{1}(\nu)-i B_{2}(\nu)+O\left(s^{-2}\right), \\
& \int_{0}^{s} t^{3} U_{3}^{2}(t, \nu)\left(\int_{0}^{t} \tau^{3} U_{1}^{2}(\tau, \nu) d \tau\right) d t \\
& =s^{4} / 4+(3 \nu+1) s^{2}+\left(30 \nu^{2}+30 \nu+11\right) \log s+C_{1}(\nu)-i C_{2}(\nu)+O\left(s^{-2}\right),
\end{aligned}
$$

where $(\nu)_{r}=\prod_{j=0}^{r-1}(\nu+j)$, and with $\Psi(z)=(\Gamma(z))^{-1} d \Gamma(z) / d z$ and $\Psi^{*}(z)=\Psi\left(z+\frac{1}{2}\right)$ $-\Psi(z)$,

$$
\begin{aligned}
& A_{1}(\nu)=2^{-1 / 2}[-8(2 \nu+1)(\Gamma(1+\nu / 2) / \Gamma((1+\nu) / 2)) \\
& \left.+\left(8 \nu^{2}+8 \nu+3\right)(\Gamma((1+\nu) / 2) / \Gamma(1+\nu / 2))\right] \sin \pi \nu, \\
& A_{2}(\nu)=2^{-1 / 2}\left[16(2 \nu+1)(\Gamma(1+\nu / 2) / \Gamma((1+\nu) / 2)) \sin ^{2} \pi \nu / 2\right. \\
& \left.+2\left(8 v^{2}+8 \nu+3\right)(\Gamma((1+\nu) / 2) / \Gamma(1+\nu / 2)) \cos ^{2} \pi \nu / 2\right], \\
& B_{1}(\nu)=-2^{-1}\left(7 \nu^{2}+4 \nu+5\right)-\left(\frac{3}{2}\right)(2 \nu+1) \cos ^{2} \pi \nu / 2 \\
& -\left(\frac{3}{2}\right)\left(2 \nu^{2}+2 \nu+1\right)\left[\log 2+\Psi((1+\nu) / 2) \cos ^{2} \pi \nu / 2+\Psi(1+\nu / 2) \sin ^{2} \pi \nu / 2\right], \\
& B_{2}(\nu)=\left(\frac{3}{4}\right)(2 \nu+1) \sin \pi \nu-\left(\frac{3}{2}\right)\left(2 \nu^{2}+2 \nu+1\right)\left(\pi+\left(\frac{1}{2}\right) \Psi^{*}((1+\nu) / 2) \sin \pi \nu\right), \\
& C_{1}(\nu)=\mathrm{R} 1\left[\int_{0}^{1} s^{3} U_{3}^{2}(s, \nu)\left(\int_{0}^{s} t^{3} U_{1}^{2}(t, \nu) d t\right) d s\right. \\
& +\int_{1}^{\infty}\left\langle s^{3} U_{3}^{2}(s, \nu)\left(\int_{0}^{s} t^{3} U_{1}^{2}(t, \nu) d t\right)-s^{3}-(6 \nu+2) s\right. \\
& \left.-\left(30 \nu^{2}+30 \nu+11\right) s^{-1}>d s-\left(3 \nu+\frac{5}{4}\right)\right], \\
& C_{2}(\nu)=-2^{-1}\left(30 \nu^{2}+30 \nu+11\right)\left(\pi+\left(\frac{1}{2}\right) \Psi^{*}((1+\nu) / 2) \sin \pi \nu\right) \\
& +(1 / 36)\left(128 \nu^{3}+192 \nu^{2}+158 \nu+47\right) \sin 2 \pi \nu \\
& +(1 / 18)\left[-\left(256 \nu^{3}+384 v^{2}+300 \nu+86\right) \cos \pi \nu\right. \\
& +\left(128 \nu^{4}+256 v^{3}+224 v^{2}+96 \nu+18\right)(\Gamma((1+\nu) / 2) / \Gamma(1+\nu / 2))^{2} \cos ^{2} \pi \nu / 2 \\
& -2\left(448 v^{2}+448 \nu+131\right)(\Gamma(1+\nu / 2) / \Gamma((1+\nu) / 2))^{2} \sin ^{2} \pi \nu / 2 \\
& +(135 / 2)(2 \nu+1)] \sin \pi v \text {, }
\end{aligned}
$$

$E_{1}(\nu)=-2^{-1}\left[\log 2+\Psi((1+\nu) / 2) \cos ^{2} \pi \nu / 2+\Psi(1+\nu / 2) \sin ^{2} \pi \nu / 2\right]$,

$E_{2}(\nu)=-2^{-1}\left(\pi+\left(\frac{1}{2}\right) \Psi^{*}((1+\nu) / 2) \sin \pi \nu\right)$.

Appendix II: Certain functions related to Bessel functions. Here we shall consider the differential equation

$$
y^{\prime \prime}(s)-s^{2} y(s)=0, \quad-\infty<s<\infty, \quad '=d / d s .
$$

Two linearly independent solutions of this equation, determined by the initial conditions $U_{1}(0)=1, U_{1}^{\prime}(0)=0, U_{2}(0)=0, U_{2}^{\prime}(0)=1$, are given by 
$U_{1}(s)=\left(\Gamma\left(\frac{3}{4}\right) / 2^{1 / 2}\right) s^{1 / 2} I_{-1 / 4}\left(s^{2} / 2\right) \quad$ for $s \geqq 0, \quad U_{1}(s)=U_{1}(-s) \quad$ for $s<0$, $U_{2}(s)=\Gamma\left(\frac{5}{4}\right)(2 s)^{1 / 2} I_{1 / 4}\left(s^{2} / 2\right) \quad$ for $s \geqq 0, \quad U_{2}(s)=-U_{2}(-s) \quad$ for $s<0$,

where $I_{\nu}(s)$ is the modified Bessel function of the first kind of order $\nu$ [14, Chapter 7]. We note that $U_{1}(s)=\left[1+s^{4} / 12+s^{8} / 672+\cdots\right], U_{2}(s)=s\left[1+s^{4} / 20+s^{8} / 1440+\cdots\right]$. For $s \geqq 0$ we also introduce the solution $U_{3}(s)=U_{1}(s) \int_{s}^{\infty} U_{1}^{-2}(t) d t=$ $\left(2 \Gamma\left(\frac{5}{4}\right) / \pi\right) s^{1 / 2} K_{1 / 4}\left(s^{2} / 2\right)$, where $K_{v}(s)$ is the modified Bessel function of the third kind of order $\nu$. We have then as $s \rightarrow \infty$ [14, Chapter 7],

$$
\begin{aligned}
& U_{1}(s)=\Gamma\left(\frac{3}{4}\right)(2 \pi s)^{-1 / 2} \exp \left(s^{2} / 2\right)\left[1+3 /(4 s)^{2}+105 / 2 !(4 s)^{4}+O\left(s^{-6}\right)\right], \\
& U_{1}^{\prime}(s)=\left(\Gamma\left(\frac{3}{4}\right) /(2 \pi)^{1 / 2}\right) s^{1 / 2} \exp \left(s^{2} / 2\right)\left[1-5 /(4 s)^{2}-135 / 2 !(4 s)^{4}+O\left(s^{-6}\right)\right], \\
& U_{2}(s)=(2 / \pi)^{1 / 2} \Gamma\left(\frac{5}{4}\right) s^{-1 / 2} \exp \left(s^{2} / 2\right)\left[1+3 /(4 s)^{2}+105 / 2 !(4 s)^{4}+O\left(s^{-6}\right)\right], \\
& U_{3}(s)=\left(2 /(\pi)^{1 / 2}\right) \Gamma\left(\frac{5}{4}\right) s^{-1 / 2} \exp \left(-s^{2} / 2\right)\left[1-3 /(4 s)^{2}+105 / 2 !(4 s)^{4}+O\left(s^{-6}\right)\right], \\
& U_{3}^{\prime}(s)=-\left(2 /(\pi)^{1 / 2}\right) \Gamma\left(\frac{5}{4}\right) s^{1 / 2} \exp \left(-s^{2} / 2\right)\left[1+5 /(4 s)^{2}-135 / 2 !(4 s)^{4}+O\left(s^{-6}\right)\right] .
\end{aligned}
$$

\section{Appendix III: Equations in integers.}

III.0. Introduction. In the investigation of the asymptotic behaviour of $\lambda_{m}(\mu)$ (see introduction) there appear certain equations in integers whose solutions lead to the determination of the coefficients in the asymptotic development of $\lambda_{m}(\mu)$. These equations arise from the relationship between the real zeros of the parabolic cylinder function and the number of zeros in $(0,1)$ of an eigenfunction of $(1.1-2)$ corresponding to $\lambda_{m}(\mu)$. Here we shall concern ourselves with stating certain results for each of three types of equations which appear in the preceding analysis (see [16]).

We shall in the sequel adopt the following notation. For $x \geqq 0, r$ and $s$ nonnegative integers, $a_{r}$ and $a_{s}$ positive numbers, let $A_{r, s}(x)$ denote the greatest positive integer less than $\left[x\left(a_{r} / a_{s}\right)^{1 / 2}+\frac{1}{2}\right]$ or zero if such a positive integer does not exist. Let $B_{r, s}(x)$ be the greatest integer less than or equal to $\left(\frac{1}{2}\right) A_{r, s}(x)$, and let $C_{r, s}(x)$ be the smallest integer greater than or equal to $\left(\frac{1}{2}\right) A_{r, s}(x)$. Finally, let

$$
\begin{aligned}
& R=\left\{x \mid x=((2 j+1) /(2 k+1))^{2}, \text { where } j \text { and } k\right. \text { are integers, } \\
& \qquad j=0, \ldots, k \text {, and } k=0,1,2, \ldots\} .
\end{aligned}
$$

III.1. The case of $p$ equations. Let $\left\{a_{r}\right\}_{r=1}^{p}, p \geqq 2$, be a set of $p$ positive numbers. For nonnegative integer $n$ put:

$$
f_{r}(n)=n+\sum_{s=1 ; s \neq r}^{p} A_{r, s}\left(n+\frac{1}{2}\right), \quad r=1, \ldots, p .
$$

THEOREM III.1.1. Let $\left(a_{r} / a_{s}\right) \notin R$ for $r, s=1, \ldots, p$ and $r \neq s$. Let $m$ be any nonnegative integer. Then there exists precisely one tuple $\left(r^{\dagger}, n^{\dagger}\right)$ such that $f_{r}+\left(n^{\dagger}\right)=m$.

For nonnegative integer $m$ let $R_{m}=\left\{x \mid x=((2 j+1) /(2 k+1))^{2}\right.$, where $j$ and $k$ are integers, $j=0, \ldots, k$, and $k=0, \ldots, m\}$.

THEOREM III.1.2. Let $m$ be a nonnegative integer. Let $\left(a_{r} / a_{s}\right) \notin R_{m}$ for $r, s=1, \ldots, p$ and $r \neq s$. Then the result of Theorem III.1.1 remains valid. 
III.2. The case of $(p+1)$ equations. Let $\left\{a_{r}\right\}_{r=0}^{p}, p \geqq 1$, be a set of $(p+1)$ positive numbers. For nonnegative integer $n$ put:

$$
\begin{aligned}
& f_{0}(n)=n+\sum_{s=1}^{p} A_{0, s}\left(2 n+\frac{3}{2}\right), \\
& f_{r}(n)=n+B_{r, 0}\left(n+\frac{1}{2}\right)+\sum_{s=1 ; s \neq r}^{p} A_{r, s}\left(n+\frac{1}{2}\right), \quad r=1, \ldots, p ; \\
& f_{0}(n)=n+\sum_{s=1}^{p} A_{0, s}\left(2 n+\frac{1}{2}\right), \\
& f_{r}(n)=n+C_{r, 0}\left(n+\frac{1}{2}\right)+\sum_{s=1 ; s \neq r}^{p} A_{r, s}\left(n+\frac{1}{2}\right), \quad r=1, \ldots, p .
\end{aligned}
$$

THEOREM III.2.1. Let $\left(a_{r} / a_{s}\right) \notin R$ for $r, s=0, \ldots, p$ and $r \neq s$. Let $m$ be any nonnegative integer. Then for each section considered above there exists precisely one tuple $\left(r^{\dagger}, n^{\dagger}\right)$ such that $f_{r}+\left(n^{\dagger}\right)=m$.

For nonnegative integer $m$ let $R_{m}=\left\{x \mid x=((2 j+1) /(2 k+1))^{2}\right.$, where $j$ and $k$ are integers, $j=0, \ldots, k$, and $k=0, \ldots,(2 m+1)\}$.

THEOREM III.2.2. Let $m$ be a nonnegative integer. Let $\left(a_{r} / a_{s}\right) \notin R_{m}$ for $r, s=0, \ldots, p$ and $r \neq s$. Then the results of Theorem III.2.1 remain valid.

III.3. The case of $(p+2)$ equations. Let $\left\{a_{r}\right\}_{r=0}^{p+1}, p \geqq 0$, be a set of $(p+2)$ positive numbers. For nonnegative integer $n$ put:

(1) $f_{0}(n)=n+B_{0, p+1}\left(2 n+\frac{3}{2}\right)+\sum_{s=1}^{p} A_{0, s}\left(2 n+\frac{3}{2}\right)$,

$$
f_{r}(n)=n+B_{r, 0}\left(n+\frac{1}{2}\right)+B_{r, p+1}\left(n+\frac{1}{2}\right)+\sum_{s=1 ; s \neq r}^{p} A_{r, s}\left(n+\frac{1}{2}\right),
$$
$r=1, \ldots, p$, if $p>0$

$$
f_{p+1}(n)=n+B_{p+1,0}\left(2 n+\frac{3}{2}\right)+\sum_{s=1}^{p} A_{p+1, s}\left(2 n+\frac{3}{2}\right) ;
$$

(2) $f_{0}(n)=n+C_{0, p+1}\left(2 n+\frac{3}{2}\right)+\sum_{s=1}^{p} A_{0, s}\left(2 n+\frac{3}{2}\right)$,

$$
\begin{aligned}
f_{r}(n)=n+B_{r, 0}\left(n+\frac{1}{2}\right)+C_{r, p+1}\left(n+\frac{1}{2}\right)+\sum_{s=1: s \neq r}^{p} A_{r, s}\left(n+\frac{1}{2}\right), \\
r=1, \ldots, p, \text { if } p>0,
\end{aligned}
$$

$$
f_{p+1}(n)=n+B_{p+1,0}\left(2 n+\frac{1}{2}\right)+\sum_{s=1}^{p} A_{p+1, s}\left(2 n+\frac{1}{2}\right) ;
$$

(3) $f_{0}(n)=n+C_{0, p+1}\left(2 n+\frac{1}{2}\right)+\sum_{s=1}^{p} A_{0, s}\left(2 n+\frac{1}{2}\right)$,

$$
\begin{aligned}
f_{r}(n) & =n+C_{r, 0}\left(n+\frac{1}{2}\right)+C_{r, p+1}\left(n+\frac{1}{2}\right)+\sum_{s=1: s \neq r}^{p} A_{r, s}\left(n+\frac{1}{2}\right), \\
r=1, \ldots, p, \quad \text { if } p>0, & \\
f_{p+1}(n) & =n+C_{p+1,0}\left(2 n+\frac{1}{2}\right)+\sum_{s=1}^{p} A_{p+1, s}\left(2 n+\frac{1}{2}\right) .
\end{aligned}
$$


TheOREM III.3.1. Let $\left(a_{r} / a_{s}\right) \notin R$ for $r, s=0, \ldots,(p+1)$ and $r \neq s$. Let $m$ be any nonnegative integer. Then for each section considered above there exists precisely one tuple $\left(r^{\dagger}, n^{\dagger}\right)$ such that $f_{r}+\left(n^{\dagger}\right)=m$.

For nonnegative integer $m$ let $R_{m}=\left\{x \mid x=((2 j+1) /(2 k+1))^{2}\right.$, where $j$ and $k$ are integers, $j=0, \ldots, k$, and $k=0, \ldots,(2 m+1)\}$.

THEOREM III.3.2. Let $m$ be a nonnegative integer. Let $\left(a_{r} / a_{s}\right) \notin R_{m}$ for $r, s=0, \ldots$, $(p+1)$ and $r \neq s$. Then the results of Theorem III.3.1 remain valid.

\section{REFERENCES}

1. R. G. D. Richardson, Theorems of oscillation for two linear differential equations of the second order, Trans. Amer. Math. Soc. 13 (1912), 22-34.

2. - Über die notwendig und hinreichenden Bedingungen für das bestehen eines Kleinschen Oszillationstheorems, Math. Ann. 73 (1912/13), 289-304.

3. M. Faierman, Boundary value problems in differential equations, Ph.D. Dissertation, University of Toronto, June 1966.

4. J. Meixner and F. W. Schäfke, Mathieusche Funktionen und Sphäroidfunktionen mit Anwendung auf physikalische und technische Problems, Die Grundlehren der math. Wissenschaften, Band 71, Springer-Verlag, Berlin, 1954. MR 16, 586.

5. M. J. O. Strutt, Reelle Eigenwerte verallgemeinerter Hillscher Eigenwertaufgaben 2. Ordnung, Math. Z. 49 (1944), 593-643. MR 6, 174.

6. R. E. Langer, The asymptotic solutions of certain linear ordinary differential equations of the second order, Trans. Amer. Math. Soc. 36 (1934), 90-106.

7. - The asymptotic solutions of ordinary linear differential equations of the second order with special reference to the Stokes phenomenon, Bull. Amer. Math. Soc. 40 (1934), 545-582.

8. - The asymptotic solutions of a linear differential equation of the second order with two turning points, Trans. Amer. Math. Soc. 90 (1959), 113-142. MR 21 \#4270.

9. N. D. Kazarinoff, Asymptotic theory of second order differential equations with two simple turning points, Arch. Rational Mech. Anal. 2 (1958), 129-150. MR 20 \#5919.

10. A. A. Dorodnicyn, Asymptotic laws of distribution of the characteristic values for certain special forms of differential equations of the second order, Uspehi Mat. Nauk 7 (1952), no. 6 (52), 3-96; English transl., Amer. Math. Soc. Transl. (2) 16 (1960), 1-101. MR 14, 876; MR 22 \#8161.

11. R. W. McKelvey, The solutions of second order linear ordinary differential equations about a turning point of order two, Trans. Amer. Math. Soc. 79 (1955), 103-123. MR 16, 1023.

12. A. Erdelyi et al., Higher transcendental functions. Vol. III, McGraw-Hill, New York, 1955. MR 16, 586.

13. E. T. Whittaker and G. N. Watson, Modern analysis, Cambridge Univ. Press, Cambridge, 1965.

14. A. Erdelyi et al., Higher transcendental functions. Vol. II, McGraw-Hill, New York, 1953. MR 15, 419.

15. M. Faierman, On a perturbation in a two-parameter ordinary differential equation of the second order, Canad. Math. Bull. 14 (1971), 25-33.

16. - Some properties of equations in integers, Canad. Math. Bull. (to appear).

17. E. C. Titchmarsh, Eigenfunction expansions associated with second order differential equations. Part I, 2nd ed., Clarendon Press, Oxford, 1962. MR 31 \#426. 
18. W. Wasow, Asymptotic expansions for ordinary differential equations, Pure and Appl. Math., vol. 14, Interscience, New York, 1965. MR 34 \#3041.

19. E. C. Titchmarsh, Eigenfunction expansions associated with second order differential equations. Part II, Clarendon Press, Oxford, 1958. MR 20 \#1065.

Department of Mathematics, Loyola College, Montreal, Quebec, Canada 